\title{
Authenticated Key Exchange from Ideal Lattices
}

\author{
Jiang Zhang ${ }^{1}$, Zhenfeng Zhang ${ }^{1, *}$, Jintai Ding ${ }^{2, *}$, Michael Snook ${ }^{2}$, and Özgür Dagdelen ${ }^{3}$ \\ 1 Institute of Software, Chinese Academy of Sciences, China \\ ${ }^{2}$ University of Cincinnati, Cincinnati, USA \\ 3 Darmstadt University of Technology, Germany \\ jiangzhang09@gmail.com,zfzhang@tca.iscas.ac.cn,jintai.ding@gmail.com, \\ snookml@mail.uc.edu, oezguer.dagdelen@ cased.de \\ * Correspoding Authors
}

\begin{abstract}
In this paper, we present a practical and provably secure two-pass AKE protocol from ideal lattices, which is conceptually simple and has similarities to the Diffie-Hellman based protocols such as HMQV (CRYPTO 2005) and OAKE (CCS 2013). Our protocol does not rely on other cryptographic primitives-in particular, it does not use signatures - simplifying the protocol and resting the security solely on the hardness of the ring learning with errors problem. The security is proven in the Bellare-Rogaway model with weak perfect forward secrecy. We also give a one-pass variant of our two-pass protocol, which might be appealing in specific applications. Several concrete choices of parameters are provided, and a proof-of-concept implementation shows that our protocols are indeed practical.
\end{abstract}

\section{Introduction}

Key Exchange (KE) is a fundamental cryptographic primitive, allowing two parties to securely generate a common secret key over an insecure network. Because symmetric cryptographic tools (e.g. AES) are reliant on both parties having a shared key in order to securely transmit data, KE is one of the most used cryptographic tools in building secure communication protocols (e.g. SSL/TLS, IPSec, SSH). Following the introduction of the Diffie-Hellman (DH) protocol [26], cryptographers have devised a wide selection of KE protocols with various use-cases. One such class is Authenticated Key Exchange (AKE), a class of KE protocols where each party is able to verify the other's identity, so that an adversary cannot impersonate one party in the conversation.

For an AKE protocol, each party has a pair of static keys: a static secret key and a corresponding static public key. The static public key is certified to belong to its owner using a public-key or ID-based infrastructure. For each run of the protocol, the parties involved generate ephemeral secret keys and use these to generate ephemeral public keys that they exchange. Then all the keys are used along with the transcripts of the session to create a shared session state, which is then passed to a key derivation function to obtain the final session key. Intuitively, such a protocol is secure if no efficient adversary is able to extract any information about the session key from the publicly exchanged messages. More formally, Bellare and Rogaway [8] introduced an indistinguishability-based security model for AKE, the BR model, which captures key authentication such as implicit mutual key authentication and confidentiality of agreed session keys. The most prominent alternatives stem from Canetti and Krawczyk [15] and LaMacchia et al.[48], that also accounts for scenarios in which the adversary is able to obtain information about a static secret key or a session state other than the state of the target session. In practice, AKE protocols are usually required to have a property, Perfect Forward Secrecy (PFS), that an adversary cannot compromise session keys after a completed session, even if it obtains the parties' static secret keys (e.g., via a heartbleed attack ${ }^{4}$ ). As shown in [46], no two-pass AKE protocol based on public-key authentication can achieve PFS. Thus, the notion of weak PFS

\footnotetext{
${ }^{4}$ http://heartbleed.com/
} 
(wPFS) is usually considered for two-pass AKE protocols, which states that the session key of an honestly run session remains private if the static keys are compromised after the session is finished [46].

One approach for achieving authentication in KE protocols is to explicitly authenticate the exchanged messages between the involved parties by using some cryptographic primitives (e.g., signatures, or MAC), which usually incurs additional computation and communication overheads with respect to the basic KE protocol, and complicates the understanding of the KE protocol. This includes several well-known protocols such as IKE [39,44], SIGMA [45], SSL [31], TLS [25,47,56,36,12], as well as the standard in German electronic identity cards, namely EAC [14,22], and the standardized protocols OPACITY [23] and PLAID [24]. Another line of designing AKEs follows the idea of MQV [57,41,46,68] (which has been standardized by ISO/IEC and IEEE, and recommended by NIST and NSA Suite B) by making good use of the algebraic structure of DH problems to achieve implicit authentication. All the above AKEs are based on classic hard problems, such as factoring, the RSA problem, or the computational/decision DH problem. Since these hard problems are vulnerable to quantum computers [64] and as we are moving into the era of quantum computing, it is very appealing to find other counterparts based on problems believed to be resistant to quantum attacks. For instance, post-quantum AKE is considered of high priority by NIST [17]. Due to the potential benefits of lattice-based constructions such as asymptotic efficiency, conceptual simplicity, and worst-case hardness assumptions, it makes perfect sense to build lattice-based AKEs.

\subsection{Our Contribution}

In this paper, we propose an efficient AKE protocol based on the Ring Learning With Errors (Ring-LWE), which in turn is as hard as some lattice problems (e.g., SIVP) in the worst case on ideal lattices [54,29]. Our method avoids introducing extra cryptographic primitives, thus simplifying the design and reducing overhead. In particular, the communicating parties are not required to either encrypt any messages with the other's public key, nor sign any of their own messages during key exchange. Furthermore, by having the key exchange as a self-contained system, we reduce the security assumptions needed, and are able to rely directly and solely on the hardness of Ring-LWE.

By utilizing many useful properties of Ring-LWE problems and discrete Gaussian distributions, we establish an approach to combine both the static and ephemeral public/secret keys, in a manner similar to HMQV [46]. Thus, our protocol not only enjoys many nice properties of HMQV such as two-pass messages, implicit key authentication, high efficiency, and without using any explicit entity authentication techniques (e.g., signatures), but also has many properties of lattice-based cryptography, such as asymptotic efficiency, conceptual simplicity, worst-case hardness assumption, as well as resistance to quantum computer attacks. However, there are also several shortcomings inherited from lattice-based cryptography, such as "handling of noises" and large public/secret keys. Besides, unlike HMQV which works on "nice-behaving" cyclic groups, the security of our protocol cannot be proven in the CK model [15] due to the underlying noisebased algebraic structures. Fortunately, we prove the security in the BR model, which is the most common model considered as it is usually strong enough for many practical applications and it comes with composability [13]. In addition, our protocol achieves weak PFS property, which is known as the best PFS notion achievable by two-pass protocols [46].

As MQV [57] and HMQV [46], we present a one-pass variant of our basic protocol (i.e., the two parties can only exchange a single message in order to derive a shared session key), which might be useful in clientserver based applications. Finally, we select concrete choices of parameters and construct a proof-of-concept implementation to examine the efficiency of our protocols. Through the implementation has not undergone any real optimization, the performance results already indicate that our protocols are practical. 
We note that none of the techniques we use prevent us from instantiating our AKE protocol based on standard lattices. One just has to keep in mind that key sizes and performance eventually become worse.

\subsection{Techniques, and Relation to HMQV}

Our AKE protocol is inspired by HMQV [46], which makes our protocol share some similarities to HMQV. However, there are also many differences between our protocol and HMQV due to the different underlying algebraic structures. To better illustrate the commons and differences between our AKE protocol and HMQV, we first briefly recall the HMQV protocol [46]. Let $\mathbb{G}$ be a cyclic group with generator $g \in \mathbb{G}$. Let $\left(P_{i}=g^{s_{i}}, s_{i}\right)$ and $\left(P_{j}=g^{s_{j}}, s_{j}\right)$ be the static public/secret key pairs of party $i$ and party $j$, respectively. During the protocol, both parties exchange ephemeral public keys, e.g., party $i$ sends $X_{i}=g^{r_{i}}$ to party $j$, and party $j$ sends $Y_{j}=g^{r_{j}}$ to party $i$. Then, both parties compute the same key material $k_{i}=\left(P_{j}^{d} Y_{j}\right)^{s_{i} c+r_{i}}=g^{\left(s_{i} c+r_{i}\right)\left(s_{j} d+r_{j}\right)}=\left(P_{i}^{c} X_{i}\right)^{s_{j} d+r_{j}}=k_{j}$ where $c=H_{1}(j, X)$ and $d=H_{1}(i, Y)$ are computed by using a function $H_{1}$, and use it as input of a key derivation function $H_{2}$ to generate a common session key, i.e., $\mathrm{sk}_{i}=H_{2}\left(k_{i}\right)=H_{2}\left(k_{j}\right)=\mathrm{sk}_{j}$.

As mentioned above, HMQV has many nice properties such as only two-pass messages, implicit key authentication, high efficiency, and without using any explicit entity authentication techniques (e.g., signatures). Our main goal is to construct a lattice-based counterpart such that it not only enjoys all those nice properties of HMQV, but also belongs to post-quantum cryptography, i.e., the underlying hardness assumption is believed to hold even against quantum computer. However, such a task is highly non-trivial since the success of HMQV extremely relies on the nice property of cyclic groups such as commutativity (i.e., $\left(g^{a}\right)^{b}=\left(g^{b}\right)^{a}$ ) and perfect (and public) randomization (i.e. $g^{a}$ can be perfectly randomized by computing $g^{a} g^{r}$ with a uniformly chosen $r$ at random).

Fortunately, as noticed in $[27,61,9]$, the Ring-LWE problem actually supports some kind of "approximate" commutativity, and can be used to build passive-secure key exchange protocol. Specifically, let $R_{q}$ be a ring, and $\chi$ be a Gaussian distribution over $R_{q}$. Then, given two Ring-LWE tuples with both secret and errors choosing from $\chi$, e.g., $\left(a, b_{1}=a s_{1}+e_{1}\right)$ and $\left(a, b_{2}=a s_{2}+e_{2}\right)$ for randomly chosen $a \leftarrow{ }_{r} R_{q}, s_{1}, s_{2}, e_{1}, e_{2} \leftarrow_{r} \chi$, the approximate equation $s_{1} b_{2} \approx s_{1} a s_{2} \approx s_{2} b_{1}$ holds with overwhelming probability for proper parameters. By the same observation, we construct an AKE protocol (as illustrated in Fig. 1), where both the static and ephemeral public keys are actually Ring-LWE elements corresponding to a globally public element $a \in R_{q}$. In order to overcome the inability of "approximate" commutativity, our protocol has to send a signal information $w_{j}$ computed by using a function Cha. Combining this with another useful function $\operatorname{Mod}_{2}$, both parties are able to compute the same key material $\sigma_{i}=\sigma_{j}$ (from the approximately equal values $k_{i}$ and $\left.k_{j}\right)$ with a guarantee that $\sigma_{j}=\operatorname{Mod}_{2}\left(k_{j}, w_{j}\right)$ has high min-entropy even conditioned on the partial information $w_{j}=\mathrm{Cha}\left(k_{j}\right)$ of $k_{j}$ (thus it can be used to derive a uniform session key $\mathrm{sk}_{j}$ ).

However, the strategy of sending out the information $w_{j}=\mathrm{Cha}\left(k_{j}\right)$ inherently brings an undesired byproduct. Specifically, unlike HMQV, the security of our AKE protocol cannot be proven in the CK model which allows the adversaries to obtain the session state $k_{j}$ via session state reveal queries. This is because in a traditional definition of session identifier that consists of all the exchanged messages, the two "different" sessions $\operatorname{sid}=\left(i, j, x_{i}, y_{j}, w_{j}\right)$ and $\operatorname{sid}^{\prime}=\left(i, j, x_{i}, y_{j}, w_{j}^{\prime}\right)$ in our protocol have the same session state, i.e., $k_{i}$ at party $i .^{5}$ This also means that we cannot directly use $\sigma_{i}=\sigma_{j}$ as the session key, because the binding between the value of $\sigma_{i}$ and the session identifier is too loose (especially for the signal part, $w_{j}$ 's). Since

\footnotetext{
${ }^{5}$ We remark that this problem might not exist if we consider a different definition of session identifier, e.g., the one that was uniquely determined at the beginning of each execution of the protocol.
} 


\begin{tabular}{|c|c|c|}
\hline Party $i$ & & Party $j$ \\
\hline $\begin{array}{l}\text { Public Key: } p_{i}=a s_{i}+2 e_{i} \in R_{q} \\
\text { Secret Key: } s_{i} \in R_{q} \\
\text { where } s_{i}, e_{i} \leftarrow_{r} \chi_{\alpha}\end{array}$ & & $\begin{array}{l}\text { Public Key: } p_{j}=a s_{j}+2 e_{j} \in R_{q} \\
\text { Secret Key: } s_{j} \in R_{q} \\
\text { where } s_{j}, e_{j} \leftarrow_{r} \chi_{\alpha}\end{array}$ \\
\hline $\begin{array}{l}x_{i}=a r_{i}+2 f_{i} \in R_{q} \\
\text { where } r_{i}, f_{i} \leftarrow r \chi_{\beta}\end{array}$ & $x_{i}$ & $\begin{array}{l}y_{j}=a r_{j}+2 f_{j} \in R_{q} \\
k_{j}=\left(p_{i} c+x_{i}\right)\left(s_{j} d+r_{j}\right)+2 c g_{j}\end{array}$ \\
\hline $\begin{array}{l}k_{i}=\left(p_{j} d+y_{j}\right)\left(s_{i} c+r_{i}\right)+2 d g_{i} \\
\text { where } g_{i} \leftarrow_{r} \chi_{\beta} \\
\sigma_{i}=\operatorname{Mod}_{2}\left(k_{i}, w_{j}\right) \in\{0,1\}^{n} \\
\operatorname{sk}_{i}=H_{2}\left(i, j, x_{i}, y_{j}, w_{j}, \sigma_{i}\right)\end{array}$ & $y_{j}, w_{j}$ & $\begin{array}{l}\text { where } r_{j}, f_{j}, g_{j} \leftarrow_{r} \chi_{\beta} \\
w_{j}=\operatorname{Cha}\left(k_{j}\right) \in\{0,1\}^{n} \\
\sigma_{j}=\operatorname{Mod}_{2}\left(k_{j}, w_{j}\right) \in\{0,1\}^{n} \\
\operatorname{sk}_{j}=H_{2}\left(i, j, x_{i}, y_{j}, w_{j}, \sigma_{j}\right)\end{array}$ \\
\hline \multicolumn{3}{|c|}{$c=H_{1}\left(i, j, x_{i}\right) \in R, d=H_{1}\left(j, i, y_{j}, x_{i}\right) \in R$} \\
\hline
\end{tabular}

Fig. 1. Our AKE protocol based on Ring-LWE, where $R_{q}=\mathbb{Z}_{q} /\left(x^{n}+1\right)$ is a ring, $\chi_{\alpha}$ and $\chi_{\beta}$ are two Gaussian distributions over $R_{q}$. The two functions Cha and $\operatorname{Mod}_{2}$ provide that $\sigma_{i}=\operatorname{Mod}_{2}\left(k_{i}, w_{j}\right)=$ $\operatorname{Mod}_{2}\left(k_{j}, w_{j}\right)=\sigma_{j}$.

both sessions sid and $\operatorname{sid}^{\prime}$ have the same session state $k_{i}$, the value $\sigma_{i}^{\prime}=\operatorname{Mod}_{2}\left(k_{i}, w_{j}^{\prime}\right)$ corresponding to sid' is simply a shift of $\sigma_{i}=\operatorname{Mod}_{2}\left(k_{i}, w_{j}\right)$ corresponding to sid (by the definition of the $\operatorname{Mod}_{2}$ function). We prevent the adversary from utilizing this weakness by setting the session key as the output of the hash function $\mathrm{H}_{2}$ (which is modeled as a random oracle) which tightly binds the session identifier sid and the key material $\sigma_{i}$ (i.e., $\mathrm{sk}_{i}=H_{2}\left(\mathrm{sid}, \sigma_{i}\right)$ ). Our technique works due to another useful property of $\mathrm{Mod}_{2}$, which guarantees that $\sigma_{i}^{\prime}=\operatorname{Mod}_{2}\left(k_{i}, w_{j}^{\prime}\right)$ preserves the high min-entropy property of $k_{i}$ for any $w_{j}^{\prime}$ (and thus is enough to generate a secure session key by the property of random oracle $\left.\mathrm{H}_{2}\right){ }^{6}{ }^{6}$

In order to finally get a security proof of our AKE protocol in the BR model with weakly perfect forward secrecy, we have to make use of the following property of Gaussian distributions namely some kind of "public randomization". Specifically, let $\chi_{\alpha}$ and $\chi_{\beta}$ be two Gaussian distributions with standard deviation $\alpha$ and $\beta$, respectively. Then, the summation of the two distributions is still a Gaussian distribution $\chi_{\gamma}$ with standard deviation $\gamma=\sqrt{\alpha^{2}+\beta^{2}}$. In particular, if $\beta \gg \alpha\left(e . g ., \beta / \alpha=2^{\omega(\log \kappa)}\right.$ for some security parameter $\kappa$ ), we have that the distribution $\chi_{\gamma}$ is statistically close to $\chi_{\beta}$. This technique is also known as "noise flooding" and has been applied, for instance, in proving robustness of the LWE assumption [37]. ${ }^{7}$ Using this technique allows to statistically hide the distribution of $\chi_{\alpha}$ in a bigger distribution $\chi_{\beta}$. The security proof of our protocol is based on this observation, and for now let us keep it in mind that a large distribution will be used to hide a small one.

To better illustrate our technique, we take party $j$ as an example, who combines his static and ephemeral secret keys by computing $\hat{r}_{j}=s_{j} d+r_{j}$ where $d=H_{1}\left(j, i, y_{j}, x_{i}\right)$. We notice that the value $\hat{r}_{j}$ actually behaves like a "signature" on the messages that party $j$ knows so far. In other words, it should be difficult to compute $\hat{r}_{j}$ if we do not know the corresponding "signing key" $s_{j}$. Indeed, this combination is necessary to provide the implicit entity authentication. However, it also posts an obstacle to get a security proof since the simulator may also be unaware of $s_{j}$. Fortunately, if the randomness $r_{j}$ is chosen from a big enough Gaussian

\footnotetext{
${ }^{6}$ We remark that this is also the reason why the nice reconciliation mechanism in [61] cannot be used in our protocol. Specifically, it is unclear whether the reconciliation function $\operatorname{rec}(\cdot, \cdot)$ in [61] could also preserve the high min-entropy property of the first input (i.e., which might not be uniformly random) for any (maliciously chosen) second input.

${ }^{7}$ Actually, noise flooding works conditioned on the size of the random variable, and thus does not require to be distributed according to $\chi_{\alpha}$.
} 
distribution, then the value $\hat{r}_{j}$ almost obliterates all information of $s_{j}$. More specifically, the simulator can directly choose $\hat{r}_{j}$ such that $\hat{r}_{j}=s_{j} d+r_{j}$ for some unknown $r_{j}$ by computing $y_{j}=\left(a \hat{r}_{j}+2 \hat{f}_{j}\right)-p_{j} d$, and programming the random oracle $d=H_{1}\left(j, i, y_{j}, x_{i}\right)$ correspondingly. Combining the properties of Gaussian distributions and the random oracle $H_{1}$, we have that $y_{j}$ is almost identically distributed as that in the real run of the protocol. Now, we check the randomness of $k_{j}=\left(p_{i} c+x_{i}\right) \hat{r}_{j}+2 c g_{j}$. Note that for the test session, we can always guarantee that at least one of $p_{i}$ and $x_{i}$ is honestly generated (and thus is computationally indistinguishable from uniformly distributed element under the Ring-LWE assumption), or else there is no "secrecy" to protect at all if both $p_{i}$ and $x_{i}$ are chosen by the adversary. That is, the value $p_{i} c+x_{i}$ is always uniformly distributed if $c$ is invertible in $R_{q}$. Again, by programming $c=H_{1}\left(i, j, x_{i}\right)$, the simulator can actually replace $p_{i} c+x_{i}$ with $\hat{x}_{i}=c^{-1} u_{i}$ for a uniformly distributed ring element $u_{i}$. In this case, we have that $k_{j}=\hat{x}_{i} \hat{r}_{j}+2 c g_{j}=c\left(u_{i} \hat{r}_{j}+2 g_{j}\right)$ should be computationally indistinguishable from a uniformly distributed element under the Ring-LWE assumption. In other words, $k_{j}$ can be used to derive a high min-entropy key material $\sigma_{j}$ as required by using the $\operatorname{Mod}_{2}$ function.

Unfortunately, directly using "noise flooding" has a significant drawback, i.e., the requirement of a super-polynomially big standard deviation $\beta$, which may lead to a nightmare for practical performance due to a super-polynomially big modulus $q$ for correctness and a very large ring dimension $n$ for the hardness of the underlying Ring-LWE problems. Fortunately, we can somehow reduce the big cost by further employing the rejection sampling technique [52]. Rejection sampling is a crucial technique in signature schemes to make the distribution of signatures independent of the signing key. Since [52] it has been applied in many other lattice-based signature schemes $[38,30,3,40]$.

In our case the combination of the static and ephemeral secret keys, $\hat{r}_{j}=s_{j} d+r_{j}$, at party $j$ is essentially a signature on all the public messages under party $j$ 's public key (we again take party $j$ as an example, but note that similar analysis also holds for party $i$ ). Thus, we can freely use the rejection sampling technique to relax the requirement on a super-polynomially big $\beta$. In other words, we can use a much smaller $\beta$, but require party $j$ to use $r_{j}$ if $\hat{r}_{j}=s_{j} d+r_{j}$ follows the distribution $\chi_{\beta}$, and to resample a new $r_{j}$ otherwise. We note that by deploying rejection sampling in our AKE it is the first time that rejection sampling is used beyond signature schemes. As for signatures, rejection sampling is done locally, and thus will not affect the interaction between the two parties, i.e., two-pass messages. Even though the computational performance of each execution might become worse with certain (small) probability (due to rejection and repeated sampling), the average computational cost is much better than the setting of using a super-polynomially big $\beta$.

\subsection{Related Work, Comparison and Discussion}

In the past few years, many cryptographers have put effort into constructing different kinds of KE protocols from lattices. At Asiacrypt 2009, Katz and Vaikuntanathan [43] proposed the first password-based authenticated key exchange protocol that can be proven secure based on the LWE assumption. Ding et al. [27] proposed a passive-secure KE protocol based on (Ring-)LWE. Like the standard DH protocol, the protocol in [27] could not provide authentication-i.e., it is not an AKE protocol-and is thus weak to man-in-themiddle attacks. Lei et al. [49] presented a KE protocol based on NTRU encryption and a new "NTRU-KE" assumption.

To the best of our knowledge, there are four papers focusing on designing AKEs from lattices [32,61,33,9]. In general, all known lattice-based AKE protocols work by following generic transformations from key encapsulation mechanisms (KEM) to AKEs and explicitly using signatures to provide authentication. Fujioka et al. [32] proposed a generic construction of AKE from KEMs, which can be proven secure in the CK model. Informally, they showed that if there is a CCA secure KEM with high min-entropy keys and a family 
Table 1. Comparison of Lattice-based AKEs (CCA* means CCA-security with high min-entropy keys [32], and EUF-CMA means existential unforgeability under chosen message attacks)

\begin{tabular}{|c|c|c|c|c|c|c|}
\hline Protocols & KEM/PKE & Signature & Message-pass & Model & RO? & Num. of $R_{q}$ \\
\hline FSXY12 [32] & CCA $^{*}$ & - & 2-pass & CK & $\times$ & $\gg 7$ \\
\hline FSXY13 [33] & OW-CCA & - & 2-pass & CK & $\sqrt{ }$ & 7 \\
\hline Peikert14 [61] & CPA & EUF-CMA & 3-pass & SK-security & $\sqrt{ }$ & $>2^{\text {a }}$ \\
\hline BCNS14 [9] & CPA & EUF-CMA & 4-pass & ACCE & $\sqrt{ }$ & $2_{\text {for KEM }}$ f $^{2}$ \\
\hline Ours & - & - & 2-pass & BR with wPFS & $\sqrt{ }$ & 2 \\
\hline
\end{tabular}

\footnotetext{
${ }^{\text {a }}$ The actual number of ring elements depends on the choice of the concrete lattice-based signatures.

${ }^{\mathrm{b}}$ Since the protocol uses traditional signatures to provide authentication, it does not contain any other ring elements.
}

of pseudorandom functions (PRF), then there is a secure AKE protocol in the standard model. Instantiated with lattice-based CCA secure KEMs such as [62,59], it is possible to construct lattice-based AKE protocols in the standard model. However, as the authors commented, their construction was just of theoretic interest due to huge public keys and the lack of an efficient and direct construction of PRFs from (Ring-)LWE. Following [32], the paper [33] tried to get a practical AKE protocol, and gave a generic construction from any one-way CCA-secure KEM in the random oracle model. The two protocols in [32,33] share some similarities such as having two-pass messages, and involving three times encryptions (i.e., two encryptions under each party's static public keys and one encryption under an ephemeral public key). For concreteness, instantiated with the CPA-secure encryption from Ring-LWE [54] (i.e., by first transforming it into a CCA-secure one using the Fujisaki-Okamoto (FO) transformation in the random oracle model), the protocol in [33] requires to exchange seven ring elements in total.

Recently, Peikert [61] presented an efficient KEM based on Ring-LWE, which was then transformed into an AKE protocol by using the same structure as SIGMA [45]. The resulting protocol involved one encryption, and two signatures and two MACs for explicit entity authentication. As the SIGMA protocol, the protocol in [61] has three-pass messages and was proven SK-secure [16] in the random oracle model. Bos et al. [9] treated Peikert's KEM as a DH-like KE protocol, and integrated it into the Transport Layer Security (TLS) protocol. Thus, their AKE protocol also employed signatures to provide explicit authentication. In fact, they used the traditional digital signatures such as RSA and ECDSA to provide authentication (i.e., it is not a pure post-quantum AKE protocol). The security of their protocol was proven in the authenticated and confidential channel establishment (ACCE) security model [42], which is based on the BR model, but has many differences to capture entity authentication and channel security.

Since the lack of concrete security analysis and parameter choices in the literature, we only give a theoretical comparison of lattice-based AKEs in Table 1. In summary, our protocol only has two-pass messages (about two ring elements) and does not use signatures/MACs at al, and its security solely relies on the hardness of Ring-LWE. To the best of our knowledge there is not a single post-quantum authenticated key exchange protocol (until this work) which solely relies on a quantum-hard computational problem and does not make use of explicit cryptographic primitives except hash functions.

\subsection{Roadmap}

In the preliminaries section, we recall the BR model and several useful tools on lattices. Then, we give a twopass AKE protocol from ideal lattices in Section 3, and prove its security based on Ring-LWE problems in 
Section 4. In Section 5, we present the one-pass variant of our protocol. The concrete choices of parameters and timings are given in Section 6.

\section{Preliminaries}

\subsection{Notation}

Let $\kappa$ be the natural security parameter, and all quantities are implicitly dependent on $\kappa$. Let poly $(\kappa)$ denote an unspecified function $f(\kappa)=O\left(\kappa^{c}\right)$ for some constant $c$. The function log denotes the natural logarithm. We use standard notation $O, \omega$ to classify the growth of functions. If $f(\kappa)=O\left(g(\kappa) \cdot \log ^{c} \kappa\right)$, we denote $f(\kappa)=\tilde{O}(g(\kappa))$. We say a function $f(\kappa)$ is negligible if for every $c>0$, there exists a $N$ such that $f(\kappa)<1 / \kappa^{c}$ for all $\kappa>N$. We use $\operatorname{negl}(\kappa)$ to denote a negligible function of $\kappa$, and we say a probability is overwhelming if it is $1-\operatorname{negl}(\kappa)$.

The set of real numbers (integers) is denoted by $\mathbb{R}$ ( $\mathbb{Z}$, resp.). We use $\leftarrow_{r}$ to denote randomly choosing an element from some distribution (or the uniform distribution over some finite set). Vectors are in column form and denoted by bold lower-case letters (e.g., $\mathbf{x})$. The $\ell_{2}$ and $\ell_{\infty}$ norms we designate by $\|\cdot\|$ and $\|\cdot\|_{\infty}$. The ring of polynomials over $\mathbb{Z}\left(\mathbb{Z}_{q}=\mathbb{Z} / q \mathbb{Z}\right.$, resp.) we denote by $\mathbb{Z}[x]\left(\mathbb{Z}_{q}[x]\right.$, resp.).

Let $X$ be a distribution over finite set $S$. The min-entropy of $X$ is defined as

$$
H_{\infty}(X)=-\log \left(\max _{s \in S} \operatorname{Pr}[X=s]\right)
$$

Intuitively, the min-entropy says that if we (privately) choose $x$ from $X$ at random, then no (unbounded) algorithm can guess the value of $x$ correctly with probability greater than $2^{-H_{\infty}(X)}$.

\subsection{Security Model for AKE}

We now recall the Bellare-Rogaway security model [8], restricted to the case of two-pass AKE protocol.

Sessions We fix a positive integer $N$ to be the maximum number of honest parties that use the AKE protocol. Each party is uniquely identified by an integer $i$ in $\{1,2, \ldots, N\}$, and has a static key pair consisting of a static secret key $\mathrm{sk}_{i}$ and static public key $\mathrm{pk}_{i}$, which is signed by a Certificate Authority (CA). A single run of the protocol is called a session. A session is activated at a party by an incoming message of the form $(\Pi, I, i, j)$ or the form $\left(\Pi, R, j, i, X_{i}\right)$, where $\Pi$ is a protocol identifier; $I$ and $R$ are role identifiers; $i$ and $j$ are party identifiers. If party $i$ receives a message of the form $(\Pi, I, i, j)$, we say that $i$ is the session initiator. Party $i$ then outputs the response $X_{i}$ intended for party $j$. If party $j$ receives a message of the form $\left(\Pi, R, j, i, X_{i}\right)$, we say that $j$ is the session responder; party $j$ then outputs a response $Y_{j}$ to party $i$. After exchanging these messages, both parties compute a session key.

If a session is activated at party $i$ with $i$ being the initiator, we associate with it a session identifier sid $=\left(\Pi, I, i, j, X_{i}\right)$ or sid $=\left(\Pi, I, i, j, X_{i}, Y_{j}\right)$. Similarly, if a session is activated at party $j$ with $j$ being the responder, the session identifier has the form $\operatorname{sid}=\left(\Pi, R, j, i, X_{i}, Y_{j}\right)$. For a session identifier sid $=(\Pi, *, i, j, *[, *])$, the third coordinate-that is, the first party identifier-is called the owner of the session; the other party is called the peer of the session. A session is said to be completed when its owner computes a session key. The matching session of sid $=\left(\Pi, I, i, j, X_{i}, Y_{j}\right)$ is the session with identifier $\widetilde{\operatorname{sid}}=\left(\Pi, R, j, i, X_{i}, Y_{j}\right)$ and vice versa. 
Adversarial Capabilities We model the adversary $\mathcal{A}$ as a probabilistic polynomial time (PPT) Turing machine with full control over all communications channels between parties, including control over session activations. In particular, $\mathcal{A}$ can intercept all messages, read them all, and remove or modify any desired messages as well as inject its own messages. We also suppose $\mathcal{A}$ is capable of obtaining hidden information about the parties, including static secret keys and session keys to model potential leakage of them in genuine protocol executions. These abilities are formalized by providing $\mathcal{A}$ with the following oracles (we split the Send query in [15] into Send ${ }_{0}$, Send $_{1}$ and Send $_{2}$ queries for the case of two-pass protocols):

- $\operatorname{Send}_{0}(\Pi, I, i, j): \mathcal{A}$ activates party $i$ as an initiator. The oracle returns a message $X_{i}$ intended for party $j$.

- $\operatorname{Send}_{1}\left(\Pi, R, j, i, X_{i}\right): \mathcal{A}$ activates party $j$ as a responder using message $X_{i}$. The oracle returns a message $Y_{j}$ intended for party $i$.

- Send ${ }_{2}\left(\Pi, R, i, j, X_{i}, Y_{j}\right): \mathcal{A}$ sends party $i$ the message $Y_{j}$ to complete a session previously activated with a $\operatorname{Send}_{0}(\Pi, I, i, j)$ query that returned $X_{i}$.

- SessionKeyReveal(sid): The oracle returns the session key associated with the session sid if it has been generated.

- Corrupt $(i)$ : The oracle returns the static secret key belonging to party $i$. A party whose key is given to $\mathcal{A}$ in this way is called dishonest; a party not compromised in this way is called honest.

- Test( $\left.\operatorname{sid}^{*}\right)$ : The oracle chooses a bit $b \leftarrow r\{0,1\}$. If $b=0$, it returns a key chosen uniformly at random; if $b=1$, it returns the session key associated with sid* ${ }^{*}$ Note that we impose some restrictions on this query. We only allow $\mathcal{A}$ to query this oracle once, and only on a fresh (see Definition 1 ) session sid*.

Definition 1 (Freshness). Let $\operatorname{sid}^{*}=\left(\Pi, I, i^{*}, j^{*}, X_{i}, Y_{j}\right)$ or $\left(\Pi, R, j^{*}, i^{*}, X_{i}, Y_{j}\right)$ be a completed session with initiator party $i^{*}$ and responder party $j^{*}$. If the matching session exists, denote it $\widetilde{\operatorname{sid}}{ }^{*}$. We say that $\operatorname{sid}^{*}$ is fresh if the following conditions all hold:

- $\mathcal{A}$ has not made a SessionKeyReveal query on sid*.

- $\mathcal{A}$ has not made a SessionKeyReveal query on sid $^{*}$ (if it exists).

- Neither party $i^{*}$ nor $j^{*}$ is dishonest if $\widetilde{\text { sid }^{*}}$ does not exist. I.e., $\mathcal{A}$ has not made a Corrupt query on either of them.

Recall that in the original BR model [8], no corruption query is allowed. In the above freshness definition, we allow the adversary to corrupt both parties of sid* if the matching session exists, i.e., the adversary can obtain the parties's secret key in advance and then passively eavesdrops the session sid* (and thus $\widetilde{s i d}^{*}$ ). We remark that this is actually stronger than what is needed for capturing wPFS [46], where the adversary is only allowed to corrupt a party after an honest session $\operatorname{sid}^{*}$ (and thus sid $^{*}$ ) has been completed.

Security Game The security of a two-pass AKE protocol is defined in terms of the following game. The adversary $\mathcal{A}$ makes any sequence of queries to the oracles above, so long as only one Test query is made on a fresh session, as mentioned above. The game ends when $\mathcal{A}$ outputs a guess $b^{\prime}$ for $b$. We say $\mathcal{A}$ wins the game if its guess is correct, so that $b^{\prime}=b$. The advantage of $\mathcal{A}, \mathbf{A d v}_{\Pi, \mathcal{A}}$, is defined as $\operatorname{Pr}\left[b^{\prime}=b\right]-1 / 2$.

Definition 2 (Security). We say that an AKE protocol $\Pi$ is secure if the following conditions hold:

- If two honest parties complete matching sessions then they compute the same session key with overwhelming probability.

- For any PPT adversary $\mathcal{A}$, the advantage $\mathbf{A d v}_{\Pi, \mathcal{A}}$ is negligible. 


\subsection{The Gaussian Distributions and Rejection Sampling}

For any positive real $\alpha \in \mathbb{R}$, and vectors $\mathbf{c} \in \mathbb{R}^{m}$, the continuous Gaussian distribution over $\mathbb{R}^{m}$ with standard deviation $\alpha$ centered at $\mathbf{v}$ is defined by the probability function $\rho_{\alpha, \mathbf{c}}(\mathbf{x})=\left(\frac{1}{\sqrt{2 \pi \sigma^{2}}}\right)^{m} \exp \left(\frac{-\|\mathbf{x}-\mathbf{v}\|^{2}}{2 \sigma^{2}}\right)$. For integer vectors $\mathbf{c} \in \mathbb{R}^{n}$, let $\rho_{s, \mathbf{c}}\left(\mathbb{Z}^{m}\right)=\sum_{\mathbf{x} \in \mathbb{Z}^{m}} \rho_{s, \mathbf{c}}(\mathbf{x})$. Then, we define the discrete Gaussian distribution over $\mathbb{Z}^{m}$ as $D_{\mathbb{Z}^{m}, s, \mathbf{c}}(\mathbf{x})=\frac{\rho_{s, \mathbf{c}}(\mathbf{x})}{\rho_{s, \mathbf{c}}\left(\mathbb{Z}^{m}\right)}$, where $\mathbf{x} \in \mathbb{Z}^{m}$. The subscripts $s$ and $\mathbf{c}$ are taken to be 1 and $\mathbf{0}$ (respectively) when omitted. The following lemma says that for large enough $\alpha$, almost all the samples from $D_{\mathbb{Z}^{m}, \alpha}$ are small.

Lemma 1 ([58]). Letting real $\alpha=\omega(\sqrt{\log m})$, constant $d>1 / \sqrt{2 \pi}$, then $\operatorname{Pr}_{\mathbf{x} \leftarrow{ }_{r} D_{\mathbb{Z}^{m}, \alpha}}[\|\mathbf{x}\|>d \cdot \alpha \sqrt{m}] \leq$ $\frac{1}{2} D^{n}$, where $D=d \sqrt{2 \pi e} \cdot e^{-\pi \cdot d^{2}}$. In particular, we have $\operatorname{Pr}_{\mathbf{x} \leftarrow r} D_{\mathbb{Z}^{m}, \alpha}[\|\mathbf{x}\|>\alpha \sqrt{m}] \leq 2^{-m+1}$.

Now, we recall rejection sampling in Theorem 1 from [52], which will be used in the security proof of our AKE protocol. Informally, the rejection sampling theorem says that for large enough $\alpha$, the distributions $D_{\mathbb{Z}^{m}, \alpha, \mathbf{c}}$ and $D_{\mathbb{Z}^{m}, \alpha}$ are statistically indistinguishable even given vector $\mathbf{c} \in \mathbb{Z}$.

Theorem 1 (Rejection Sampling [52]). Let $V$ be a subset of $\mathbb{Z}^{m}$ in which all the elements have norms less than $T, \alpha=\omega(T \sqrt{\log m})$ be a real, and $\psi: V \rightarrow \mathbb{R}$ be a probability distribution. Then there exists a constant $M=O(1)$ such that the distribution of the following algorithm Samp $\mathrm{S}_{1}$ :

1: $\mathbf{c} \leftarrow r \psi$

2: $\mathbf{z} \leftarrow{ }_{r} D_{\mathbb{Z}^{m}, \alpha, \mathbf{c}}$

3: output $(\mathbf{z}, \mathbf{c})$ with probability $\min \left(\frac{D_{\mathbb{Z}^{m}, \alpha}(\mathbf{z})}{M D_{\mathbb{Z}^{m}, \alpha, \mathbf{c}}(\mathbf{z})}, 1\right)$.

is within statistical distance $\frac{2^{-\omega(\log m)}}{M}$ of the distribution of the following algorithm $\mathrm{Samp}_{2}$ :

1: $\mathbf{c} \leftarrow r \psi$

2: $\mathbf{z} \leftarrow{ }_{r} D_{\mathbb{Z}^{m}, \alpha}$

3: output $(\mathbf{z}, \mathbf{c})$ with probability $1 / M$.

Moreover, the probability that $\mathrm{Samp}_{1}$ outputs something is at least $\frac{1-2^{-\omega(\log m)}}{M}$. More concretely, if $\alpha=\tau T$ for any positive $\tau$, then $M=e^{12 / \tau+1 /\left(2 \tau^{2}\right)}$ and the output of algorithm Samp $\mathrm{P}_{1}$ is within statistical distance $\frac{2^{-100}}{M}$ of the output of $\mathrm{Samp}_{2}$, and the probability that $\mathcal{A}$ outputs something is at least $\frac{1-2^{-100}}{M}$.

\subsection{Ring Learning with Errors}

Let the integer $n$ be a power of 2 , and consider the ring $R=\mathbb{Z}[x] /\left(x^{n}+1\right)$. For any positive integer $q$, we define the ring $R_{q}=\mathbb{Z}_{q}[x] /\left(x^{n}+1\right)$ analogously. For any polynomial $y(x)$ in $R$ (or $R_{q}$ ), we identify $y$ with its coefficient vector in $\mathbb{Z}^{n}$ (or $\mathbb{Z}_{q}^{n}$ ). Then we define the norm of a polynomial to be the norm of its coefficient vector.

Lemma 2. For any $s, t \in R$, we have $\|s \cdot t\| \leq \sqrt{n} \cdot\|s\| \cdot\|t\|$ and $\|s \cdot t\|_{\infty} \leq n \cdot\|s\|_{\infty} \cdot\|t\|_{\infty}$.

Besides, the discrete Gaussian distribution over the ring $R$ can be naturally defined as the distribution of ring elements whose coefficient vectors are distributed according to the discrete Gaussian distribution over $\mathbb{Z}^{n}$, e.g., $D_{\mathbb{Z}^{n}, \alpha}$ for some positive real $\alpha$. Letting $\chi_{\alpha}$ be the discrete Gaussian distribution over $\mathbb{Z}^{n}$ with standard deviation $\alpha$ centered at $\mathbf{0}$, i.e., $\chi_{\alpha}:=D_{\mathbb{Z}^{n}, \alpha}$, we now adopt the following notational convention: since bold-face letters denote vectors, $\mathbf{x} \leftarrow_{r} \chi_{\alpha}$ means we sample the vector $\mathbf{x}$ from the distribution $\chi_{\alpha}$; for 
normal weight variables (e.g. $y \leftarrow_{r} \chi_{\alpha}$ ) we sample an element of $R$ whose coefficient vector is distributed according to $\chi_{\alpha}$.

Now we come to the statement of the Ring-LWE assumption; we will use a special case detailed in [54]. Let $R_{q}$ be defined as above, and $s \leftarrow_{r} R_{q}$. We define $A_{s, \chi_{\alpha}}$ to be the distribution of the pair $(a, a s+x) \in$ $R_{q} \times R_{q}$, where $a \leftarrow_{r} R_{q}$ is uniformly chosen and $x \leftarrow_{r} \chi_{\alpha}$ is independent of $a$.

Definition 3 (Ring-LWE Assumption). Let $R_{q}$ and $\chi_{\alpha}$ be defined as above, and let $s \leftarrow_{r} R_{q}$. The Ring$L W E$ assumption $R L W E_{q, \alpha}$ states that it is hard for any PPT algorithm to distinguish $A_{s, \chi_{\alpha}}$ from the uniform distribution on $R_{q} \times R_{q}$ with only polynomially many samples.

The following lemma says that the hardness of the Ring-LWE assumption can be reduced to some hard lattice problems such as the Shortest Independet Vectors Problem (SIVP) over ideal lattices.

Proposition 1 (A special case of [54]). Let $n$ be a power of 2, let $\alpha$ be a real number in $(0,1)$, and $q$ a prime such that $q \bmod 2 n=1$ and $\alpha q>\omega(\sqrt{\log n})$. Define $R_{q}=\mathbb{Z}_{q}[x] /\left\langle x^{n}+1\right\rangle$ as above. Then there exists a polynomial time quantum reduction from $\tilde{O}(\sqrt{n} / \alpha)$-SIVP in the worst case to average-case $R L W E_{q, \beta}$ with samples, where $\beta=\alpha q \cdot(n \ell / \log (n \ell))^{1 / 4}$.

It has been proven that the Ring-LWE assumption still holds even if the secret $s$ is chosen according to the error distribution $\chi_{\beta}$ rather than uniformly [1,54]. This variant is known as the normal form, and is preferable for controlling the size of the error term [11,10]. The underlying Ring-LWE assumption also holds when scaling the error by a constant $t$ relatively prime to $q$ [11], i.e., using the pair $\left(a_{i}, a_{i} s+t x_{i}\right)$ rather than $\left(a_{i}, a_{i} s+x_{i}\right)$. Several lattice-based cryptographic schemes have been constructed based on this variant $[11,10]$. In our case, we will fix $t=2$. Besides, recall that the $\operatorname{RLWE}_{q, \beta}$ assumption guarantees that for some prior fixed (but randomly chosen) $s$, the tuple $(a, a s+2 x)$ is computationally indistinguishable from the uniform distribution over $R_{q} \times R_{q}$ if $a \leftarrow_{r} R_{q}$ and $x \leftarrow \chi_{\beta}$. In this paper, we will use a matrix form ring-LWE assumption. Formally, let $B_{\chi_{\beta}, \ell_{1}, \ell_{2}}$ be the distribution of $\left(\mathbf{a}, \mathbf{B}=\left(b_{i, j}\right)\right) \in R_{q}^{\ell_{1}} \times R_{q}^{\ell_{1} \times \ell_{2}}$, where $\mathbf{a}=\left(a_{0}, \ldots, a_{\ell_{1}-1}\right) \leftarrow_{r} R_{q}^{\ell_{1}}, \mathbf{s}=\left(s_{0}, \ldots, s_{\ell_{2}-1}\right) \leftarrow_{r} R_{q}^{\ell_{2}}, e_{i, j} \leftarrow_{r} \chi_{\beta}$, and $b_{i, j}=a_{i} s_{j}+2 e_{i, j}$ for $i \in\left\{0, \ldots, \ell_{1}-1\right\}$ and $j \in\left\{0, \ldots, \ell_{2}-1\right\}$. For polynomially bounded $\ell_{1}$ and $\ell_{2}$, one can show that the distribution of $B_{\chi_{\beta}, \ell_{1}, \ell_{2}}$ is pseudorandom based on the $\operatorname{RLWE}_{q, \beta}$ assumption [62].

\section{Authenticated Key Exchange from Ring-LWE}

We now introduce some notation before presenting our protocol. For odd prime $q>2$, denote $\mathbb{Z}_{q}=$ $\left\{-\frac{q-1}{2}, \ldots, \frac{q-1}{2}\right\}$ and define the subset $E:=\left\{-\left\lfloor\frac{q}{4}\right\rfloor, \ldots,\left\lfloor\frac{q}{4}\right\rceil\right\}$ as the middle half of $\mathbb{Z}_{q}$. We also define Cha to be the characteristic function of the complement of $E$, so Cha $(v)=0$ if $v \in E$ and 1 otherwise. Obviously, for any $v$ in $\mathbb{Z}_{q}, v+\operatorname{Cha}(v) \cdot \frac{q-1}{2} \bmod q$ belongs to $E$. We define an auxiliary modular function, $\operatorname{Mod}_{2}: \mathbb{Z}_{q} \times\{0,1\} \rightarrow\{0,1\}:$

$$
\operatorname{Mod}_{2}(v, b)=\left(v+b \cdot \frac{q-1}{2}\right) \bmod q \bmod 2 .
$$

In the following lemma, we show that given the bit $b=\mathrm{Cha}(v)$, and a value $w=v+2 e$ with sufficiently small $e$, we can recover $\operatorname{Mod}_{2}(v$, Cha $(v))$. In particular, we have $\operatorname{Mod}_{2}(v, b)=\operatorname{Mod}_{2}(w, b)$.

Lemma 3. Let $q$ be an odd prime, $v \in \mathbb{Z}_{q}$ and $e \in \mathbb{Z}_{q}$ such that $|e|<q / 8$. Then, for $w=v+2 e$, we have $\operatorname{Mod}_{2}(v, \mathrm{Cha}(v))=\operatorname{Mod}_{2}(w, C h a(v))$. 
Proof. Note that $w+\mathrm{Cha}(v) \frac{q-1}{2} \bmod q=v+\mathrm{Cha}(v) \frac{q-1}{2}+2 e \bmod q$. Now, $v+\operatorname{Cha}(v) \frac{q-1}{2} \bmod q$ is in $E$ as we stated above; that is, $-\left\lfloor\frac{q}{4}\right\rfloor \leq v+\operatorname{Cha}(v) \frac{q-1}{2} \bmod q \leq\left\lfloor\frac{q}{4}\right\rceil$. Thus, since $-q / 8<e<q / 8$, we have $-\left\lfloor\frac{q}{2}\right\rfloor \leq v+\mathrm{Cha}(v) \frac{q-1}{2} \bmod q+2 e \leq\left\lfloor\frac{q}{2}\right\rceil$. Therefore, we have $v+\operatorname{Cha}(v) \frac{q-1}{2} \bmod q+2 e=$ $v+\operatorname{Cha}(v) \frac{q-1}{2}+2 e \bmod q=w+\operatorname{Cha}(v) \frac{q-1}{2} \bmod q . \operatorname{Thus}_{,} \operatorname{Mod}_{2}(w, \operatorname{Cha}(v))=\operatorname{Mod}_{2}(v, \operatorname{Cha}(v))$.

Now, we extend the functions Cha and $\operatorname{Mod}_{2}$ to ring $R_{q}$ by applying them coefficient-wise to ring elements. Namely, for ring element $v=\left(v_{0}, \ldots, v_{n-1}\right) \in R_{q}$ and binary-vector $\mathbf{b}=\left(b_{0}, \ldots, b_{n-1}\right) \in\{0,1\}^{n}$, define $\widetilde{\operatorname{Cha}}(v)=\left(\operatorname{Cha}\left(v_{0}\right), \ldots, \operatorname{Cha}\left(v_{n-1}\right)\right)$ and $\widetilde{\operatorname{Mod}_{2}}(v, \mathbf{b})=\left(\operatorname{Mod}_{2}\left(v_{0}, b_{0}\right), \ldots, \operatorname{Mod}_{2}\left(v_{n-1}, b_{n-1}\right)\right)$. For simplicity, we slightly abuse the notations and still use Cha and $\operatorname{Mod}_{2}$ to denote $\widetilde{\text { Cha }}$ and $\widetilde{\operatorname{Mod}_{2}}$, respectively. Clearly, the result in Lemma 3 still holds when extending to ring elements.

In our AKE protocol, the two involved parties will use Cha and $\mathrm{Mod}_{2}$ to derive a common key material. Concretely, the responder will publicly send the result of Cha on his own secret ring element to the initiator in order to compute a shared key material from two "closed" ring elements (by applying the $\mathrm{Mod}_{2}$ function). Ideally, for uniformly chosen element $v$ from $R_{q}$ at random, we hope that the output of $\operatorname{Mod}_{2}(v, \operatorname{Cha}(v))$ is uniformly distributed $\{0,1\}^{n}$. However, this can never happen when $q$ is a odd prime. Fortunately, we can show that the output of $\operatorname{Mod}_{2}(v, \mathrm{Cha}(v))$ conditioned on Cha $(v)$ has high min-entropy, thus can be used to extract an (almost) uniformly session key. Actually, we can prove a stronger result.

Lemma 4. Let $q$ be any odd prime and $R_{q}$ be the ring defined above. Then, for any $\mathbf{b} \in\{0,1\}^{n}$ and any $v^{\prime} \in$ $R_{q}$, the output distribution of $\operatorname{Mod}_{2}\left(v+v^{\prime}, \mathbf{b}\right)$ given Cha $(v)$ has min-entropy at least $-n \log \left(\frac{1}{2}+\frac{1}{|E|-1}\right)$, where $v$ is uniformly chosen from $R_{q}$ at random. In particular, when $q>203$, we have $-n \log \left(\frac{1}{2}+\frac{1}{|E|-1}\right)>$ $0.97 n$.

Proof. Since each coefficient of $v$ is independently and uniformly chosen from $\mathbb{Z}_{q}$ at random, we can simplify the proof by focusing on the first coefficient of $v$. Formally, letting $v=\left(v_{0}, \ldots, v_{n-1}\right), v^{\prime}=\left(v_{0}^{\prime}, \ldots, v_{n-1}^{\prime}\right)$ and $\mathbf{b}=\left(b_{0}, \ldots, b_{n-1}\right)$, we condition on $\operatorname{Cha}\left(v_{0}\right)$ :

- If Cha $\left(v_{0}\right)=0$, then $v_{0}+v_{0}^{\prime}+b_{0} \cdot \frac{q-1}{2}$ is uniformly distributed over $v_{0}^{\prime}+b_{0} \cdot \frac{q-1}{2}+E \bmod q$. This shifted set has $(q+1) / 2$ elements, which are either consecutive integers-if the shift is small enoughor two sets of consecutive integers-if the shift is large enough to cause wrap-around. Thus, we must distinguish a few cases:

- If $|E|$ is even and no wrap-around occurs, then the result of $\operatorname{Mod}_{2}\left(v_{0}+v_{0}^{\prime}, b_{0}\right)$ is clearly uniform on $\{0,1\}$. Namely, the result of $\operatorname{Mod}_{2}\left(v_{0}+v_{0}^{\prime}, b_{0}\right)$ has no bias.

- If $|E|$ is odd and no wrap-around occurs, then the result of $\operatorname{Mod}_{2}\left(v_{0}+v_{0}^{\prime}, b_{0}\right)$ has a bias with probability $\frac{1}{2|E|}$ over $\{0,1\}$. In other words, the $\operatorname{Mod}_{2}\left(v_{0}+v_{0}^{\prime}, b_{0}\right)$ will output either 0 or 1 with probability exactly $\frac{1}{2}+\frac{1}{2|E|}$.

- If $|E|$ is odd and wrap-around does occur, then the set $v_{0}^{\prime}+b_{0} \cdot \frac{q-1}{2}+E \bmod q$ splits into two parts, one with an even number of elements, and one with an odd number of elements. This leads to the same situation as with no wrap-around.

- If $|E|$ is even and wrap-around occurs, then our sample space is split into either two even-sized sets, or two odd sized sets. If both are even, then once again the result of $\operatorname{Mod}_{2}\left(v_{0}+v_{0}^{\prime}, b_{0}\right)$ is uniform. If both are odd, it is easy to calculate that the result of $\operatorname{Mod}_{2}\left(v_{0}+v_{0}^{\prime}, b_{0}\right)$ has a bias with probability $\frac{1}{|E|}$ over $\{0,1\}$.

- If Cha $\left(v_{0}\right)=1, v_{0}+v_{0}^{\prime}+b_{0} \cdot \frac{q-1}{2}$ is uniformly distributed over $v_{0}^{\prime}+b_{0} \cdot \frac{q-1}{2}+\tilde{E}$, where $\tilde{E}=\mathbb{Z}_{q} \backslash E$. Now $|\tilde{E}|=|E|-1$, so by splitting into the same cases as $\operatorname{Cha}\left(v_{0}\right)=0$, the result of $\operatorname{Mod}_{2}\left(v_{0}+v_{0}^{\prime}, b\right)$ has a bias with probability $\frac{1}{|E|-1}$ over $\{0,1\}$. 
In all, we have that the result of $\operatorname{Mod}_{2}\left(v_{0}+v_{0}^{\prime}, b_{0}\right)$ conditioned on Cha $\left(v_{0}\right)$ has min-entropy at least $-\log \left(\frac{1}{2}+\frac{1}{|E|-1}\right)$. Since the bits in the result of $\operatorname{Mod}_{2}\left(v+v^{\prime}, \mathbf{b}\right)$ are independent, we have that given Cha $(v)$, the min-entropy $H_{\infty}\left(\operatorname{Mod}_{2}\left(v+v^{\prime}, \mathbf{b}\right)\right) \geq-n \log \left(\frac{1}{2}+\frac{1}{|E|-1}\right)$. This completes the first claim. The second claim directly follows from the fact that $-\log \left(\frac{1}{2}+\frac{1}{|E|-1}\right)>-\log (0.51)>0.97$ when $q>203$.

Remark 1 (On Uniformly Distributed Keys). It is known that randomness extractor can be used to obtain an almost uniformly distributed key from a biased bit-string with high min-entropy $[19,66,67,28,4]$. In practice, as recommended by NIST [5], one can actually use the standard cryptographic hash functions such as SHA2 to derive a uniformly distributed key if the source string has at least $2 \kappa$ min-entropy, where $\kappa$ is the length of the cryptographic hash function.

\subsection{The Protocol}

We now describe our protocol in detail. Let $n$ be a power of 2 , and $q$ be an odd prime such that $q$ mod $2 n=$ 1. Take $R=\mathbb{Z}[x] /\left(x^{n}+1\right)$ and $R_{q}=\mathbb{Z}_{q}[x] /\left(x^{n}+1\right)$ as above. For $\gamma \in \mathbb{R}^{+}$, let $H_{1}:\{0,1\}^{*} \rightarrow \chi_{\gamma}=D_{\mathbb{Z}^{n}, \gamma}$ be a hash function that always output invertible elements in $R_{q}{ }^{8}$ Let $H_{2}:\{0,1\}^{*} \rightarrow\{0,1\}^{\kappa}$ be the key derivation function, where $\kappa$ is the bit-length of the final shared key. We model both functions as random oracles [7]. Let $\chi_{\alpha}, \chi_{\beta}$ be two discrete Gaussian distributions with parameters $\alpha, \beta \in \mathbb{R}^{+}$. Let $a \in R_{q}$ be the global public parameter uniformly chosen from $R_{q}$ at random, and $M$ be a constant determined by Theorem 1. Let $p_{i}=a s_{i}+2 e_{i} \in R_{q}$ be party $i$ 's static public key, where $\left(s_{i}, e_{i}\right)$ is the corresponding static secret key; both $s_{i}$ and $e_{i}$ are taken from the distribution $\chi_{\alpha}$. Similarly, party $j$ has static public key $p_{j}=a s_{j}+2 e_{j}$ and static secret key $\left(s_{j}, e_{j}\right)$.

Initiation Party $i$ proceeds as follows:

1. Sample $r_{i}, f_{i} \leftarrow_{r} \chi_{\beta}$ and compute $x_{i}=a r_{i}+2 f_{i}$;

2. Compute $c=H_{1}\left(i, j, x_{i}\right), \hat{r}_{i}=s_{i} c+r_{i}$ and $\hat{f}_{i}=e_{i} c+f_{i}$;

3. Letting $\mathbf{z} \in \mathbb{Z}^{2 n}$ be the coefficient vector of $\hat{r}_{i}$ concatenated with the coefficient vector of $\hat{f}_{i}$, and $\mathbf{z}_{1} \in \mathbb{Z}^{2 n}$ be the coefficient vector of $s_{i} c$ concatenated with the coefficient vector of $e_{i} c$, repeat the steps $1 \sim 3$ with probability $1-\min \left(\frac{D_{\mathbb{Z}^{2 n}, \beta}(\mathbf{z})}{M D_{\mathbb{Z}^{2 n}, \beta, \mathbf{z}_{1}}(\mathbf{z})}, 1\right)$.

4. Send $x_{i}$ to party $j$.

Response After receiving $x_{i}$ from party $i$, party $j$ proceeds as follows:

$1^{\prime}$. Sample $r_{j}, f_{j} \leftarrow_{r} \chi_{\beta}$ and compute $y_{j}=a r_{j}+2 f_{j}$;

$2^{\prime}$. Compute $d=H_{1}\left(j, i, y_{j}, x_{i}\right), \hat{r}_{j}=s_{j} d+r_{j}$ and $\hat{f}_{j}=e_{j} d+f_{j}$;

$3^{\prime}$. Letting $\mathbf{z} \in \mathbb{Z}^{2 n}$ be the coefficient vector of $\hat{r}_{j}$ concatenated with the coefficient vector of $\hat{f}_{j}$, and $\mathbf{z}_{1} \in \mathbb{Z}^{2 n}$ be the coefficient vector of $s_{j} d$ concatenated with the coefficient vector of $e_{j} d$, repeat the steps $1^{\prime} \sim 3^{\prime}$ with probability $1-\min \left(\frac{D_{\mathbb{Z}^{2 n}, \beta}(\mathbf{z})}{M D_{\mathbb{Z}^{2 n}, \beta, \mathbf{z}_{1}}(\mathbf{z})}, 1\right)$;

$4^{\prime}$. Sample $g_{j} \leftarrow_{r} \chi_{\beta}$ and compute $k_{j}=\left(p_{i} c+x_{i}\right) \hat{r}_{j}+2 c g_{j}$ where $c=H_{1}\left(i, j, x_{i}\right)$;

$5^{\prime}$. Compute $w_{j}=\operatorname{Cha}\left(k_{j}\right) \in\{0,1\}^{n}$ and send $\left(y_{j}, w_{j}\right)$ to party $i$;

$6^{\prime}$. Compute $\sigma_{j}=\operatorname{Mod}_{2}\left(k_{j}, w_{j}\right)$ and derive the session key sk $\mathrm{sk}_{j}=H_{2}\left(i, j, x_{i}, y_{j}, w_{j}, \sigma_{j}\right)$.

Finish Party $i$ receives the pair $\left(y_{j}, w_{j}\right)$ from party $j$, and proceeds as follows:

\footnotetext{
${ }^{8}$ In practice, one can first use a hash function such as SHA-2 to obtain a uniformly random string, and then use it to sample from $D_{\mathbb{Z}^{n}, \gamma}$. The algorithm output a sample only if it is invertible in $R_{q}$, otherwise, it tries another sample and repeats. By Lemma 10 in [65], we can have a good probability to sample an invertible element in each trial for an appropriate choice of $\gamma$.
} 
5. Sample $g_{i} \leftarrow_{r} \chi_{\beta}$ and compute $k_{i}=\left(p_{j} d+y_{j}\right) \hat{r}_{i}+2 d g_{i}$ where $d=H_{1}\left(j, i, y_{j}, x_{i}\right)$;

6. Compute $\sigma_{i}=\operatorname{Mod}_{2}\left(k_{i}, w_{j}\right)$ and derive the session $\operatorname{key~sk}_{i}=H_{2}\left(i, j, x_{i}, y_{j}, w_{j}, \sigma_{i}\right)$.

In the above protocol, both parties will make use of rejection sampling, i.e., they will repeat the first three steps with certain probability. By Theorem 1, the probability that each party will repeat the steps with probability about $\frac{1}{M}$ for some constant $M$ and appropriately chosen $\beta$. Thus, one can hope that both parties will send something to each other after an averaged $M$ times repetitions of the first three steps. In the following subsection, we will show that once they send something to each other, both parties will finally compute a shared session key.

\subsection{Correctness}

To show the correctness of our AKE protocol, i.e., that both parties compute the same session $\mathrm{key} \mathrm{sk}_{i}=\mathrm{sk}_{j}$, it suffices to show that $\sigma_{i}=\sigma_{j}$. Since $\sigma_{i}$ and $\sigma_{j}$ are both the output of $\operatorname{Mod}_{2}$ with $\operatorname{Cha}\left(k_{j}\right)$ as the second argument, we need only to show that $k_{i}$ and $k_{j}$ are sufficiently close by Lemma 3. Note that the two parties will compute $k_{i}$ and $k_{j}$ as follows:

$$
\begin{aligned}
k_{i} & =\left(p_{j} d+y_{j}\right) \hat{r}_{i}+2 d g_{i} & k_{j} & =\left(p_{i} c+x_{i}\right) \hat{r}_{j}+2 c g_{j} \\
& =a\left(s_{j} d+r_{j}\right) \hat{r}_{i}+2\left(e_{j} d+f_{j}\right) \hat{r}_{i}+2 d g_{i} & & =a\left(s_{i} c+r_{i}\right) \hat{r}_{j}+2\left(e_{i} c+f_{i}\right) \hat{r}_{j}+2 c g_{j} \\
& =a \hat{r}_{i} \hat{r}_{j}+2 \widetilde{g}_{i} & & =a \hat{r}_{i} \hat{r}_{j}+2 \widetilde{g}_{j}
\end{aligned}
$$

where $\widetilde{g}_{i}=\hat{f}_{j} \hat{r}_{i}+d g_{i}$, and $\widetilde{g}_{j}=\hat{f}_{i} \hat{r}_{j}+c g_{j}$. Then $k_{i}=k_{j}+2\left(\widetilde{g}_{i}-\widetilde{g}_{j}\right)$, and we have $\sigma_{i}=\sigma_{j}$ if $\left\|\widetilde{g}_{i}-\widetilde{g}_{j}\right\|_{\infty}<q / 8$ by Lemma 3 .

\section{Security}

Theorem 2. Let $n$ be a power of 2 satisfying $0.97 n \geq 2 \kappa$, prime $q>203$ satisfying $q=1 \bmod 2 n$, $\beta=\omega(\alpha \gamma n \sqrt{n \log n})$. Then, if $R L W E_{q, \alpha}$ is hard, the proposed AKE is secure with respect to Definition 2 in the random oracle model.

The intuition behind our proof is quite simple. Since the public element $a$ and the public key of each party (e.g., $p_{i}=a s_{i}+2 e_{i}$ ) actually consist of a $\operatorname{RLWE}_{q, \alpha}$ tuple with Gaussian parameter $\alpha$ (scaled by 2), the parties' static public keys are computationally indistinguishable from uniformly distributed elements in $R_{q}$ under the Ring-LWE assumption. Similarly, both the exchanged elements $x_{i}$ and $y_{j}$ are also computationally indistinguishable from uniformly distributed elements in $R_{q}$ under the $\operatorname{RLWE}_{q, \beta}$ assumption.

Without loss of generality, we take party $j$ as an example to check the distribution of the session key. Note that if $k_{j}$ is uniformly distributed over $R_{q}$, we have $\sigma_{j} \in\{0,1\}^{n}$ has high min-entropy even conditioned on $w_{j}$ by Lemma 4 (e.g., $\left.0.97 n>2 \kappa\right)$. Since $H_{2}$ is a random oracle, we have that $\mathrm{sk}_{j}$ is uniformly distributed over $\{0,1\}^{\kappa}$ as expected. Now, let's check the distribution of $k_{j}=\left(p_{i} c+x_{i}\right)\left(s_{j} d+r_{j}\right)+2 c g_{j}$. As one can imagine, we want to establish the randomness of $k_{j}$ based on pseudorandomness of "Ring-LWE samples" with public element $\hat{a}_{j}=c^{-1}\left(p_{i} c+x_{i}\right)=p_{i}+c^{-1} x_{i}$, the secret $\hat{s}_{j}=s_{j} d+r_{j}$, as well as the error term $2 g_{j}$ (thus we have $\left.k_{j}=c\left(\hat{a}_{j} \hat{s}_{j}+2 g_{j}\right)\right)$. Actually, $k_{j}$ is pseudorandom due to the following fact: 1$) c$ is invertible in $R_{q}$;2) $\hat{a}_{j}$ is uniformly distributed over $R_{q}$ whenever $p_{i}$ or $x_{i}$ is uniform, and $\hat{s}_{j}$ has distribution statistically close to $\chi_{\beta}$ by the strategy of rejection sampling in Theorem 1 . In other words, $\hat{a}_{j} \hat{s}_{j}+2 g_{j}$ is statistically close to a $\operatorname{RLWE}_{q, \beta}$ sample, and thus is pseudorandom.

Formally, let $N$ be the maximum number of parties, and $m$ be maximum number of sessions for each party. We distinguish the following five types of adversaries: 
Type I: $\operatorname{sid}^{*}=\left(\Pi, I, i^{*}, j^{*}, x_{i^{*}},\left(y_{j^{*}}, w_{j^{*}}\right)\right)$ is the test session, and $y_{j^{*}}$ is output by a session activated at party $j$ by a $\operatorname{Send}_{1}\left(\Pi, R, j^{*}, i^{*}, x_{i^{*}}\right)$ query.

Type II: $\operatorname{sid}^{*}=\left(\Pi, I, i^{*}, j^{*}, x_{i^{*}},\left(y_{j^{*}}, w_{j^{*}}\right)\right)$ is the test session, and $y_{j^{*}}$ is not output by a session activated at party $j^{*}$ by a $\operatorname{Send}_{1}\left(\Pi, R, j^{*}, i^{*}, x_{i^{*}}\right)$ query.

Type III: $\operatorname{sid}^{*}=\left(\Pi, R, j^{*}, i^{*}, x_{i^{*}},\left(y_{j^{*}}, w_{j^{*}}\right)\right)$ is the test session, and $x_{i^{*}}$ is not output by a session activated at party $i^{*}$ by a $\operatorname{Send}_{0}\left(\Pi, I, i^{*}, j^{*}\right)$ query.

Type IV: $\operatorname{sid}^{*}=\left(\Pi, R, j^{*}, i^{*}, x_{i^{*}},\left(y_{j^{*}}, w_{j^{*}}\right)\right)$ is the test session, and $x_{i^{*}}$ is output by a session activated at party $i^{*}$ by a $\operatorname{Send}_{0}\left(\Pi, I, i^{*}, j^{*}\right)$ query, but $i^{*}$ either never completes the session, or $i^{*}$ completes it with exact $y_{j^{*}}$.

Type V: $\operatorname{sid}^{*}=\left(\Pi, R, j^{*}, i^{*}, x_{i^{*}},\left(y_{j^{*}}, w_{j^{*}}\right)\right)$ is the test session, and $x_{i^{*}}$ is output by a session activated at party $i^{*}$ by a $\operatorname{Send}_{0}\left(\Pi, I, i^{*}, j^{*}\right)$ query, but $i^{*}$ completes the session with another $y_{j}^{\prime} \neq y_{j^{*}}$.

The five types of adversaries give a complete partition of all the adversaries. The weak perfect forward secrecy (wPFS) is captured by allowing Type I and Type IV adversaries to obtain the static secret keys of both party $i^{*}$ and $j^{*}$ by using Corrupt queries. Since $\operatorname{sid}^{*}$ definitely has no matching session for Type II, Type III, and Type $\mathbf{V}$ adversaries, no corruption to either party $i^{*}$ or party $j^{*}$ is allowed by Definition 1 . The security proofs for the five types of adversaries are similar, except the forking lemma [6] is involved for Type II, Type III, and Type $\mathbf{V}$ adversaries by using the assumption that $H_{1}$ is a random oracle. Informally, the adversary must first "commit" $x_{i}$ (or $y_{j}$ ) before seeing $c$ (or $d$ ), thus it cannot determine the value $p_{i} c+x_{i}$ (or $p_{j} d+y_{i}$ ) in advance (but the simulator can determine the values by programming $H_{1}$ when it tries to embed Ring-LWE instances with respect to either $p_{i} c+x_{i}$ or $p_{j} d+y_{i}$ as discussed before).

\subsection{Type I Adversary}

In this subsection, we prove that our AKE is secure against any PPT Type I adversary $\mathcal{A}$.

Lemma 5. Let $n$ be a power of 2 satisfying $0.97 n \geq 2 \kappa$, prime $q>203$ satisfying $q=1 \bmod 2 n$, $\beta=\omega(\alpha \gamma n \sqrt{n \log n})$. Then, if $R L W E_{q, \alpha}$ is hard, the proposed AKE is secure against any PPT Type I adversary $\mathcal{A}$ in the random oracle model.

Proof. We prove this lemma via a sequence of games $G_{1, l}$ for $0 \leq l \leq 7$. Boxes are used to highlight the changes of each game with respect to its previous game.

Game $G_{1,0} \mathcal{S}$ chooses $i^{*}, j^{*} \leftarrow r\{1, \ldots, N\}, s_{i^{*}}, s_{j^{*}} \leftarrow_{r}\{1, \ldots, m\}$, and hopes that the adversary will use $\operatorname{sid}^{*}=\left(\Pi, I, i^{*}, j^{*}, x_{i^{*}},\left(y_{j^{*}}, w_{j^{*}}\right)\right)$ as the test session, where $x_{i^{*}}$ is output by the $s_{i^{*}}$-th session of party $i^{*}$, and $y_{j^{*}}$ is output by the $s_{j}^{*}$-th session of party $j^{*}$ activated by a $\operatorname{Send}_{1}\left(\Pi, R, j^{*}, i^{*}, x_{i^{*}}\right)$ query. Then, $\mathcal{S}$ chooses $a \leftarrow_{r} R_{q}$, generates static public keys for all parties (by choosing $s_{i}, e_{i} \leftarrow_{r} \chi_{\alpha}$ ), and simulates the security game for $\mathcal{A}$. Specifically, $\mathcal{S}$ maintains two tables $L_{1}, L_{2}$ for the random oracles $H_{1}, H_{2}$, respectively, and answers the queries from $\mathcal{A}$ as follows:

- $H_{1}($ in $)$ : If there doesn't exist a tuple $($ in, out $)$ in $L_{1}$, choose an element out $\leftarrow_{r} \chi_{\gamma}$, and add (in,out) into $L_{1}$. Then, return out to $\mathcal{A}$.

- $H_{2}($ in $)$ queries: If there doesn't exist a tuple $($ in,$o u t)$ in $L_{2}$, choose a vector out $\leftarrow_{r}\{0,1\}^{\kappa}$, and add (in, out) into $L_{2}$. Then, return out to $\mathcal{A}$.

- $\operatorname{Send}_{0}(\Pi, I, i, j): \mathcal{A}$ activates a new session of $i$ with intended party $j, \mathcal{S}$ proceeds as follows:

1. Sample $r_{i}, f_{i} \leftarrow_{r} \chi_{\beta}$ and compute $x_{i}=a r_{i}+2 f_{i}$;

2. Compute $c=H_{1}\left(i, j, x_{i}\right), \hat{r}_{i}=s_{i} c+r_{i}$ and $\hat{f}_{i}=e_{i} c+f_{i}$; 
3. Letting $\mathbf{z} \in \mathbb{Z}^{2 n}$ be the coefficient vector of $\hat{r}_{i}$ concatenated with the coefficient vector of $\hat{f}_{i}$, and $\mathbf{z}_{1} \in \mathbb{Z}^{2 n}$ be the coefficient vector of $s_{i} c$ concatenated with the coefficient vector of $e_{i} c$, repeat the steps $1 \sim 3$ with probability $1-\min \left(\frac{D_{\mathbb{Z}^{2 n}, \beta}(\mathbf{z})}{M D_{\mathbb{Z}^{2 n}, \beta, \mathbf{z}_{1}}(\mathbf{z})}, 1\right)$.

4. Return $x_{i}$ to $\mathcal{A}$;

- $\operatorname{Send}_{1}\left(\Pi, R, j, i, x_{i}\right): \mathcal{S}$ proceeds as follows:

$1^{\prime}$. Sample $r_{j}, f_{j} \leftarrow_{r} \chi_{\beta}$ and compute $y_{j}=a r_{j}+2 f_{j}$;

$2^{\prime}$. Compute $d=H_{1}\left(j, i, y_{j}, x_{i}\right), \hat{r}_{j}=s_{j} d+r_{j}$ and $\hat{f}_{j}=e_{j} d+f_{j}$;

$3^{\prime}$. Letting $\mathbf{z} \in \mathbb{Z}^{2 n}$ be the coefficient vector of $\hat{r}_{j}$ concatenated with the coefficient vector of $\hat{f}_{j}$, and $\mathbf{z}_{1} \in \mathbb{Z}^{2 n}$ be the coefficient vector of $s_{j} d$ concatenated with the coefficient vector of $e_{j} d$, repeat the steps $1^{\prime} \sim 3^{\prime}$ with probability $1-\min \left(\frac{D_{\mathbb{Z}^{2 n}, \beta}(\mathbf{z})}{M D_{\mathbb{Z}^{2 n}, \beta, \mathbf{z}_{1}}(\mathbf{z})}, 1\right)$;

$4^{\prime}$. Sample $g_{j} \leftarrow_{r} \chi_{\beta}$ and compute $k_{j}=\left(p_{i} c+x_{i}\right) \hat{r}_{j}+2 c g_{j}$ where $c=H_{1}\left(i, j, x_{i}\right)$;

$5^{\prime}$. Compute $w_{j}=\mathrm{Cha}\left(k_{j}\right) \in\{0,1\}^{n}$ and return $\left(y_{j}, w_{j}\right)$ to $\mathcal{A}$;

$6^{\prime}$. Compute $\sigma_{j}=\operatorname{Mod}_{2}\left(k_{j}, w_{j}\right)$ and derive the session $\operatorname{key~sk}_{j}=H_{2}\left(i, j, x_{i}, y_{j}, w_{j}, \sigma_{j}\right)$.

- $\operatorname{Send}_{2}\left(\Pi, I, i, j, x_{i},\left(y_{j}, w_{j}\right)\right): \mathcal{S}$ computes $k_{i}$ and $\mathrm{sk}_{i}$ as follows:

5. Sample $g_{i} \leftarrow_{r} \chi_{\beta}$ and compute $k_{i}=\left(p_{j} d+y_{j}\right) \hat{r}_{i}+2 d g_{i}$ where $d=H_{1}\left(j, i, y_{j}, x_{i}\right)$;

6. Compute $\sigma_{i}=\operatorname{Mod}_{2}\left(k_{i}, w_{j}\right)$ and derive the session $\operatorname{key~sk}_{i}=H_{2}\left(i, j, x_{i}, y_{j}, w_{j}, \sigma_{i}\right)$.

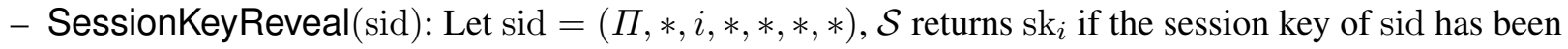
generated.

- Corrupt $(i)$ : Return the static secret key $s_{i}$ of $i$ to $\mathcal{A}$.

- Test(sid): Let sid $=\left(\Pi, I, i, j, x_{i},\left(y_{j}, w_{j}\right)\right), \mathcal{S}$ aborts if $(i, j) \neq\left(i^{*}, j^{*}\right)$, or $x_{i}$ and $y_{j}$ are not output by the $s_{i^{*}}$-th session of $i^{*}$ and the $s_{j}^{*}$-th session of $j^{*}$, respectively. Else, $\mathcal{S}$ chooses $b \leftarrow{ }_{r}\{0,1\}$, returns $s k_{i}^{\prime} \leftarrow_{r}\{0,1\}^{\kappa}$ if $b=0$. Otherwise, return the session key $\mathrm{sk}_{i}$ of sid.

Claim 1 The probability that $\mathcal{S}$ will not abort in $G_{1,0}$ is at least $\frac{1}{m^{2} N^{2}}$.

Proof. This claim directly follows from the fact that $\mathcal{S}$ randomly chooses $i^{*}, j^{*} \leftarrow{ }_{r}\{1, \ldots, N\}$ and $s_{i^{*}}, s_{j}^{*} \leftarrow r\{1, \ldots, m\}$ independently from the view of $\mathcal{A}$.

Game $G_{1,1} \mathcal{S}$ behaves almost the same as in $G_{1,0}$ except in the following case:

- $\operatorname{Send}_{1}\left(\Pi, R, j, i, x_{i}\right):$ If $(i, j) \neq\left(i^{*}, j^{*}\right)$, or it is not the $s_{j}^{*}$-th session of $j^{*}, \mathcal{S}$ answers the query as in Game $G_{1,0}$. Otherwise, it proceeds as follows:

$1^{\prime}$. Sample $r_{j}, f_{j} \leftarrow_{r} \chi_{\beta}$ and compute $y_{j}=a r_{j}+2 f_{j}$;

$2^{\prime}$. Sample an invertible element $d \leftarrow_{r} \chi_{\gamma}$, compute $\hat{r}_{j}=s_{j} d+r_{j}$ and $\hat{f}_{j}=e_{j} d+f_{j}$;

$3^{\prime}$. Letting $\mathbf{z} \in \mathbb{Z}^{2 n}$ be the coefficient vector of $\hat{r}_{j}$ concatenated with the coefficient vector of $\hat{f}_{j}$, and $\mathbf{z}_{1} \in \mathbb{Z}^{2 n}$ be the coefficient vector of $s_{j} d$ concatenated with the coefficient vector of $e_{j} d$, repeat the steps $1^{\prime} \sim 3^{\prime}$ with probability $1-\min \left(\frac{D_{\mathbb{Z}^{2 n}, \beta}(\mathbf{z})}{M D_{\mathbb{Z}^{2 n}, \beta, \mathbf{z}_{1}}(\mathbf{z})}, 1\right)$;

4'. Abort if there is a tuple $\left(\left(j, i, y_{j}, x_{i}\right), *\right)$ in $L_{1}$. Else, add $\left(\left(j, i, y_{j}, x_{i}\right), d\right)$ into $L_{1}$. Then, sample $g_{j} \leftarrow_{r}$ $\chi_{\beta}$ and compute $k_{j}=\left(p_{i} c+x_{i}\right) \hat{r}_{j}+2 c g_{j}$ where $c=H_{1}\left(i, j, x_{i}\right)$;

$5^{\prime}$. Compute $w_{j}=\operatorname{Cha}\left(k_{j}\right) \in\{0,1\}^{n}$ and return $\left(y_{j}, w_{j}\right)$ to $\mathcal{A}$;

$6^{\prime}$. Compute $\sigma_{j}=\operatorname{Mod}_{2}\left(k_{j}, w_{j}\right)$ and derive the session $\operatorname{key~sk}_{j}=H_{2}\left(i, j, x_{i}, y_{j}, w_{j}, \sigma_{j}\right)$.

In the following, we denote $F_{1, l}$ as the event that $\mathcal{A}$ outputs a guess $b^{\prime}$ that equals to $b$ in Game $G_{1, l}$. 
Claim 2 If $R L W E_{q, \beta}$ is hard, then $\operatorname{Pr}\left[F_{1, l}\right]=\operatorname{Pr}\left[F_{1,0}\right]-\operatorname{negl}(\kappa)$.

Proof. Since $H_{1}$ is a random oracle, Game $G_{1,0}$ and Game $G_{1,1}$ are identical if the adversary $\mathcal{A}$ does not make a $H_{1}$ query $\left(\left(j, i, y_{j}, x_{i}\right), *\right)$ before $\mathcal{S}$ generates $y_{j}$. Thus, the claim follows if the probability that $\mathcal{A}$ makes such a query in both Games is negligible. Actually, if $\mathcal{A}$ can make the query before seeing $y_{j}$ with non-negligible probability, we can construct an algorithm $\mathcal{B}$ that breaks the $\operatorname{RLWE}_{q, \beta}$ assumption.

Formally, after given a ring-LWE challenge tuple $(a, \mathbf{b}) \in R_{q} \times R_{q}^{\ell}$ in matrix form for some polynomially bounded $\ell, \mathcal{B}$ sets $a=u$ and behaves like in Game $G_{1,0}$ until $\mathcal{B}$ has to generate $y_{j}$ for the $s_{j}^{*}$-th session of $j^{*}$ intended for party $i^{*}$. Instead of generating a fresh $y_{j}, \mathcal{B}$ simply sets $y_{j}$ as the first unused elements in $\mathbf{b}=\left(b_{0}, \ldots, b_{\ell-1}\right)$, and checks if there is a tuple $\left(\left(j, i, y_{j}, x_{i}\right), *\right)$ in $L_{1}$. If yes, it returns 1 and aborts, else it returns 0 and aborts.

It is easy to check that $\mathcal{A}$ has the same view as in $G_{1,0}$ and $G_{1,1}$ until the point that $\mathcal{B}$ has to compute $y_{j}$. Moreover, if $\mathbf{b}=\left(b_{0}=u s_{0}+2 x_{0}, \ldots, b_{\ell}=u s_{\ell}+2 x_{\ell}\right)$ for some randomly choose $s, x \leftarrow_{r} \chi_{\beta}$, we have the probability that $\mathcal{A}$ will make the $H_{1}$ query with $\left(j, i, y_{j}, x_{i}\right)$ is non-negligible by assumption. While if $\mathbf{b}$ is uniformly distributed over $\mathbb{R}_{q}^{\ell}$, we have the probability that $\mathcal{A}$ will make the $H_{1}$ query with $\left(j, i, y_{j}, x_{i}\right)$ is negligible. This shows that $\mathcal{B}$ can be used to solve Ring-LWE assumption by interacting with $\mathcal{A}$.

Game $G_{1,2} \mathcal{S}$ behaves almost the same as in $G_{1,1}$ except in the following case:

- $\operatorname{Send}_{1}\left(\Pi, R, j, i, x_{i}\right)$ : If $(i, j) \neq\left(i^{*}, j^{*}\right)$, or it is not the $s_{j}^{*}$-th session of $j^{*}, \mathcal{S}$ answers the query as in Game $G_{1,1}$. Otherwise, it proceeds as follows:

$1^{\prime}$. Sample an invertible element $d \leftarrow_{r} \chi_{\gamma}$, and $\mathbf{z} \leftarrow{ }_{r} D_{\mathbb{Z}^{2 n}, \beta}$;

$2^{\prime}$. Interpreting $\mathbf{z}$ as two ring elements $\hat{r}_{j}, \hat{f}_{j} \in R_{q}$, and define $y_{j}=a \hat{r}_{j}+2 \hat{f}_{j}-p_{j} d$.

$3^{\prime}$. Repeat the steps $1^{\prime} \sim 3^{\prime}$ with probability $1-1 / M$;

$4^{\prime}$. Abort if there is a tuple $\left(\left(j, i, y_{j}, x_{i}\right), *\right)$ in $L_{1}$. Else, add $\left(\left(j, i, y_{j}, x_{i}\right), d\right)$ into $L_{1}$. Then, sample $g_{j} \leftarrow_{r} \chi_{\beta}$ and compute $k_{j}=\left(p_{i} c+x_{i}\right) \hat{r}_{j}+2 c g_{j}$ where $c=H_{1}\left(i, j, x_{i}\right)$

$5^{\prime}$. Compute $w_{j}=\mathrm{Cha}\left(k_{j}\right) \in\{0,1\}^{n}$ and return $\left(y_{j}, w_{j}\right)$ to $\mathcal{A}$;

$6^{\prime}$. Compute $\sigma_{j}=\operatorname{Mod}_{2}\left(k_{j}, w_{j}\right)$ and derive the session $\operatorname{key~sk}_{j}=H_{2}\left(i, j, x_{i}, y_{j}, w_{j}, \sigma_{j}\right)$.

Claim 3 If $\beta=\omega(\alpha \gamma n \sqrt{n \log n})$, then $\operatorname{Pr}\left[F_{1,2}\right]=\operatorname{Pr}\left[F_{1,1}\right]-\operatorname{negl}(\kappa)$.

Proof. By Lemma 1 and Lemma 2, we have that both $\left\|s_{j} d\right\| \leq \alpha \gamma n \sqrt{n}$ and $\left\|e_{j} d\right\| \leq \alpha \gamma n \sqrt{n}$ (in Game $\left.G_{1,1}\right)$ hold with overwhelming probability. This means that $\beta=\omega(\alpha \gamma n \sqrt{n \log n})$ satisfies the requirement in Theorem 1, and thus the distribution of $(d, \mathbf{z})$ in Game $G_{1,2}$ is statistically close to that in $G_{1,1}$. The claims follows from the fact that the equation $y_{j}=a \hat{r}_{j}+2 \hat{f}_{j}-p_{j} d$ holds in both Game $G_{1,1}$ and $G_{1,2}$.

Game $G_{1,3} \mathcal{S}$ behaves almost the same as in $G_{1,2}$, except for the following case:

- $\operatorname{Send}_{0}(\Pi, I, i, j)$ : If $(i, j) \neq\left(i^{*}, j^{*}\right)$, or it is not the $s_{i^{*}}$-th session of $i^{*}, \mathcal{S}$ answers as in Game $G_{1,2}$. Otherwise, it proceeds as follows:

1. Sample $r_{i}, f_{i} \leftarrow_{r} \chi_{\beta}$ and compute $x_{i}=a r_{i}+2 f_{i}$;

2. Sample an invertible element $c \leftarrow_{r} \chi_{\gamma}$, compute $\hat{r}_{i}=s_{i} c+r_{i}$ and $\hat{f}_{i}=e_{i} c+f_{i}$;

3. Letting $\mathbf{z} \in \mathbb{Z}^{2 n}$ be the coefficient vector of $\hat{r}_{i}$ concatenated with the coefficient vector of $\hat{f}_{i}$, and $\mathbf{z}_{1} \in \mathbb{Z}^{2 n}$ be the coefficient vector of $s_{i} c$ concatenated with the coefficient vector of $e_{i} c$, repeat the steps $1 \sim 3$ with probability $1-\min \left(\frac{D_{\mathbb{Z}^{2 n}, \beta}(\mathbf{z})}{M D_{\mathbb{Z}^{2 n}, \beta, \mathbf{z}_{1}}(\mathbf{z})}, 1\right)$. 
4. Abort if there is a tuple $\left(\left(i, j, x_{i}\right), *\right)$ in $L_{1}$. Else, add $\left(\left(i, j, x_{i}\right), c\right)$ into $L_{1}$. Return $x_{i}$ to $\mathcal{A}$.

Claim 4 If $R L W E_{q, \beta}$ is hard, then $\operatorname{Pr}\left[F_{1,3}\right]=\operatorname{Pr}\left[F_{1,2}\right]-\operatorname{negl}(\kappa)$.

Proof. The proof is similar to the proof of Claim 2, we omit the details.

Game $G_{1,4} \mathcal{S}$ behaves almost the same as in $G_{1,3}$ except for the following case:

- $\operatorname{Send}_{0}(\Pi, I, i, j)$ : If $(i, j) \neq\left(i^{*}, j^{*}\right)$, or it is not the $s_{i^{*}}$-th session of $i^{*}, \mathcal{S}$ answers as in Game $G_{1,3}$. Otherwise, it proceeds as follows:

1. Sample an invertible element $c \leftarrow_{r} \chi_{\gamma}$, and $\mathbf{z} \leftarrow{ }_{r} D_{\mathbb{Z}^{2 n}, \beta}$;

2. Interpreting $\mathbf{z}$ as two ring elements $\hat{r}_{i}, \hat{f}_{i} \in R_{q}$, and define $x_{i}=a \hat{r}_{i}+2 \hat{f}_{i}-p_{i} c$.

3. Repeat the steps $1 \sim 3$ with probability $1-1 / M$;

4. Abort if there is a tuple $\left(\left(i, j, x_{i}\right), *\right)$ in $L_{1}$. Else, add $\left(\left(i, j, x_{i}\right), c\right)$ into $L_{1}$. Return $x_{i}$ to $\mathcal{A}$.

Claim 5 If $\beta=\omega(\alpha \gamma n \sqrt{n \log n})$, then $\operatorname{Pr}\left[F_{1,4}\right]=\operatorname{Pr}\left[F_{1,3}\right]-\operatorname{negl}(\kappa)$.

Proof. The proof is similar to the proof of Claim 3, we omit the details.

Game $G_{1,5} \mathcal{S}$ behaves almost the same as in $G_{1,4}$ except for the following case:

- $\operatorname{Send}_{2}\left(\Pi, I, i, j, x_{i},\left(y_{j}, w_{j}\right)\right)$ : If $(i, j) \neq\left(i^{*}, j^{*}\right)$, or it is not the $s_{i^{*}}$-th session of $i^{*}, \mathcal{S}$ behaves as in Game $G_{1,4}$. Otherwise, if $\left(y_{j}, w_{j}\right)$ is output by the $s_{j}^{*}$-th session of party $j^{*}, \mathcal{S}$ sets $\operatorname{sk}_{i}=\mathrm{sk}_{j}$, where $\mathrm{sk}_{j}$ is the session key of sid $=\left(\Pi, R, j, i, x_{i},\left(y_{j}, w_{j}\right)\right)$. Else, $\mathcal{S}$ samples $g_{i} \leftarrow_{r} \chi_{\beta}$ and computes $k_{i}=\left(p_{j} d+y_{j}\right) \hat{r}_{i}+2 d g_{i}$ where $d=H_{1}\left(j, i, y_{j}, x_{i}\right)$. Finally, it computes $\sigma_{i}=\operatorname{Mod}_{2}\left(k_{i}, w_{j}\right)$ and derive the session $\operatorname{key~sk}_{i}=H_{2}\left(i, j, x_{i}, y_{j}, w_{j}, \sigma_{i}\right)$.

Claim $6 \operatorname{Pr}\left[F_{1,5}\right]=\operatorname{Pr}\left[F_{1,4}\right]-\operatorname{negl}(\kappa)$.

Proof. This claim follows since $G_{1,5}$ is just a conceptual change of $G_{1,4}$ by the correctness of the protocol.

Game $G_{1,6} \mathcal{S}$ behaves almost the same as in $G_{1,5}$ except in the following case:

- $\operatorname{Send}_{0}(\Pi, I, i, j)$ : If $(i, j) \neq\left(i^{*}, j^{*}\right)$, or it is not the $s_{i^{*}}$-th session of $i^{*}, \mathcal{S}$ answers as in Game $G_{1,5}$. Otherwise, it proceeds as follows:

1. Sample an invertible element $c \leftarrow_{r} \chi_{\gamma}$, and $\hat{x}_{i} \leftarrow{ }_{r} R_{q}$;

2. Define $x_{i}=\hat{x}_{i}-p_{i} c$.

3. Repeat the steps $1 \sim 3$ with probability $1-1 / M$;

4. Abort if there is a tuple $\left(\left(i, j, x_{i}\right), *\right)$ in $L_{1}$. Else, add $\left(\left(i, j, x_{i}\right), c\right)$ into $L_{1}$. Return $x_{i}$ to $\mathcal{A}$.

- $\operatorname{Send}_{2}\left(\Pi, I, i, j, x_{i},\left(y_{j}, w_{j}\right)\right)$ : If $(i, j) \neq\left(i^{*}, j^{*}\right)$, or it is not the $s_{i^{*}}$-th session of $i^{*}$, or $\left(y_{j}, w_{j}\right)$ is output by the $s_{j}^{*}$-th session of party $j^{*}, \mathcal{S}$ behaves the same as in $G_{1,5}$. Otherwise, it proceeds as follows:

5. Randomly choose $k_{i} \leftarrow_{r} R_{q}$;

6. Compute $\sigma_{i}=\operatorname{Mod}_{2}\left(k_{i}, w_{j}\right)$ and derive the session key sk $\mathrm{sk}_{i}=H_{2}\left(i, j, x_{i}, y_{j}, w_{j}, \sigma_{i}\right)$. 
Note that in Game $G_{1,6}$, we have made two changes: 1$)$ The term $a \hat{r}_{i}+2 \hat{f}_{i}$ in Game $G_{1,5}$ is replaced by a uniformly chosen element $\hat{x} \in R_{q}$ at random; 2) The value $k_{i}=\left(p_{j} d+y_{j}\right) \hat{r}_{i}+2 d g_{i}$ in Game $G_{1,5}$ is replaced by a uniformly chosen string $k_{i} \leftarrow_{r} R_{q}$, when $\left(y_{j}, w_{j}^{\prime}\right)$ is output by the $s_{j}^{*}$-th session of party $j^{*}$ but $w_{j} \neq w_{j}^{\prime}$. In the following, we will employ the "deferred analysis" proof technique in [34], which informally allows us to proceed the security games by patiently postponing some tough probability analysis to a later game. Specially, for $\ell=5,6,7$, denote $Q_{1, l}$ as the event in Game $G_{1, \ell}$ that 1$)\left(y_{j}, w_{j}^{\prime}\right)$ is output by the $s_{j}^{*}$-th session of party $j^{*}$ but $w_{j} \neq w_{j}^{\prime}$, and 2) $\mathcal{A}$ makes a query to $H_{2}$ that is exactly used to generate the session key sk $k_{i}$ for the $s_{i^{*}}$-th session of party $i^{*}$, i.e., $\operatorname{sk}_{i}=H_{1}\left(i, j, x_{i}, y_{j}, w_{j}, \sigma_{i}\right)$ for $\sigma_{i}=\operatorname{Mod}_{2}\left(k_{i}, w_{j}\right)$. Ideally, if $Q_{1,5}$ does not happen, then the adversary cannot distinguish whether a correctly computed $k_{i}$ or a randomly chosen one is used (since $\mathrm{H}_{2}$ is a random oracle, and the adversary gains no information about $k_{i}$ even if it obtains the session key sk ${ }_{i}$. However, we cannot prove the claim immediately due to technical reason. Instead, we will show that $\operatorname{Pr}\left[Q_{1,5}\right] \approx \operatorname{Pr}\left[Q_{1,6}\right] \approx \operatorname{Pr}\left[Q_{1,7}\right]$ and $\operatorname{Pr}\left[Q_{1,7}\right]$ is negligible in $\kappa$.

Claim 7 If $R L W E_{q, \beta}$ is hard, $\operatorname{Pr}\left[Q_{1,6}\right]=\operatorname{Pr}\left[Q_{1,5}\right]-\operatorname{negl}(\kappa)$, and $\operatorname{Pr}\left[F_{1,6} \mid \neg Q_{1,6}\right]=\operatorname{Pr}\left[F_{1,5} \mid \neg Q_{1,5}\right]-$ $\operatorname{negl}(\kappa)$.

Proof. Note that $H_{2}$ is a random oracle, the event $Q_{1,5}$ is independent from the distribution of the corresponding $\mathrm{sk}_{i}$. Namely, no matter whether or not $\mathcal{A}$ obtains sk, $\operatorname{Pr}\left[Q_{1,5}\right]$ is the same, which also holds for $\operatorname{Pr}\left[Q_{1,6}\right]$. In addition, under the $\operatorname{RLWE}_{q, \beta}$ assumption, we have $\hat{x}_{i}=a \hat{r}_{i}+2 \hat{f}_{i}$ in $G_{1,5}$ is computationally indistinguishable from uniform distribution over $R_{q}$, and thus the public information (i.e., static public keys and public transcripts) in $G_{1,5}$ and $G_{1,6}$ is computationally indistinguishable. In particular, the view of the adversary $\mathcal{A}$ before $Q_{1, \ell}$ happens for $\ell=5,6$ is computationally indistinguishable, which implies that $\operatorname{Pr}\left[Q_{1,6}\right]=\operatorname{Pr}\left[Q_{1,5}\right]-\operatorname{negl}(\kappa)$. Besides, if $Q_{1, l}$ for $l=5,6$ does not happen, the distribution of sk $_{i}$ is the same in both games. In other words, $\operatorname{Pr}\left[F_{1,6} \mid \neg Q_{1,6}\right]=\operatorname{Pr}\left[F_{1,5} \mid \neg Q_{1,5}\right]-\operatorname{negl}(\kappa)$.

Game $G_{1,7} \mathcal{S}$ behaves almost the same as in $G_{1,6}$ except in the following case:

- $\operatorname{Send}_{1}\left(\Pi, R, j, i, x_{i}\right):$ If $(i, j) \neq\left(i^{*}, j^{*}\right)$, or it is not the $s_{j}^{*}$-th session of $j^{*}, \mathcal{S}$ answers the query as in Game $G_{1,6}$. Otherwise, it proceeds as follows:

$1^{\prime}$. Sample an invertible element $d \leftarrow_{r} \chi_{\gamma}$, and $\hat{y}_{j} \leftarrow_{r} R_{q}$;

$2^{\prime}$. Define $y_{j}=\hat{y}_{j}-p_{j} d$.

$3^{\prime}$. Repeat the steps $1^{\prime} \sim 3^{\prime}$ with probability $1-1 / M$;

$4^{\prime}$. Abort if there is a tuple $\left(\left(j, i, y_{j}, x_{i}\right), *\right)$ in $L_{1}$. Else, add $\left(\left(j, i, y_{j}, x_{i}\right), d\right)$ into $L_{1}$. Then, the simulator $\mathcal{S}$ uniformly chooses $k_{j} \leftarrow_{r} R_{q}$ at random;

$5^{\prime}$. Compute $w_{j}=\operatorname{Cha}\left(k_{j}\right) \in\{0,1\}^{n}$ and return $\left(y_{j}, w_{j}\right)$ to $\mathcal{A}$;

$6^{\prime}$. Compute $\sigma_{j}=\operatorname{Mod}_{2}\left(k_{j}, w_{j}\right)$ and derive the session key sk $\mathrm{s}_{j}=H_{2}\left(i, j, x_{i}, y_{j}, w_{j}, \sigma_{j}\right)$.

Claim 8 Let $n$ be a power of 2 , prime $q>203$ satisfying $q=1 \bmod 2 n, \beta=\omega(\alpha \gamma n \sqrt{n \log n})$. Then, if $R L W E_{q, \beta}$ is hard, Game $G_{1,6}$ and $G_{1,7}$ are computationally indistinguishable. In particular, we have $\operatorname{Pr}\left[Q_{1,7}\right]=\operatorname{Pr}\left[Q_{1,6}\right]-\operatorname{negl}(\kappa)$, and $\operatorname{Pr}\left[F_{1,7} \mid \neg Q_{1,7}\right]=\operatorname{Pr}\left[F_{1,6} \mid \neg Q_{1,6}\right]-\operatorname{negl}(\kappa)$.

Proof. Assume there is an adversary that distinguishes Game $G_{1,6}$ and $G_{1,7}$, we now construct a distinguisher $\mathcal{D}$ that solves the Ring-LWE problem. Specifically, let $\left(\mathbf{u}=\left(u_{0}, \ldots, u_{\ell-1}\right), \mathbf{B}\right) \in R_{q}^{\ell} \times R_{q}^{\ell \times \ell}$ be a challenge Ring-LWE tuple in matrix form for some polynomially bounded $\ell, \mathcal{D}$ first sets public parameter $a=u_{0}$. Then, it randomly chooses invertible elements $\mathbf{v}=\left(v_{1}, \ldots, v_{\ell-1}\right) \leftarrow \chi_{\gamma}^{\ell-1}$, and compute $\hat{\mathbf{u}}=\left(v_{1} \cdot u_{1}, \ldots, v_{\ell-1} u_{\ell-1}\right)$. Finally, $\mathcal{D}$ behaves the same as $\mathcal{S}$ in Game $G_{1,6}$, except for the following cases: 
- $\operatorname{Send}_{0}(\Pi, I, i, j)$ : If $(i, j) \neq\left(i^{*}, j^{*}\right)$, or it is not the $s_{i^{*}}$-th session of $i^{*}, \mathcal{S}$ answers as in Game $G_{1,5}$. Otherwise, it proceeds as follows:

1. Set $c$ and $\hat{x}_{i}$ be the first unused element in $\mathbf{v}$ and $\hat{\mathbf{u}}$, respectively;

2. Define $x_{i}=\hat{x}_{i}-p_{i} c$.

3. Repeat the steps $1 \sim 3$ with probability $1-1 / M$;

4. Abort if there is a tuple $\left(\left(i, j, x_{i}\right), *\right)$ in $L_{1}$. Else, add $\left(\left(i, j, x_{i}\right), c\right)$ into $L_{1}$. Return $x_{i}$ to $\mathcal{A}$.

- $\operatorname{Send}_{1}\left(\Pi, R, j, i, x_{i}\right)$ : If $(i, j) \neq\left(i^{*}, j^{*}\right)$, or it is not the $s_{j}^{*}$-th session of $j^{*}, \mathcal{S}$ answers the query as in Game $G_{1,6}$. Otherwise, it proceeds as follows:

$1^{\prime}$. Sample an invertible element $d \leftarrow_{r} \chi_{\gamma}$, and set $\hat{y}_{j}$ be the first unused element in $\mathbf{b}_{0}=\left(b_{0,0}, \ldots, b_{0, \ell-1}\right)$;

$2^{\prime}$. Define $y_{j}=\hat{y}_{j}-p_{j} d$.

$3^{\prime}$. Repeat the steps $1^{\prime} \sim 3^{\prime}$ with probability $1-1 / M$;

$4^{\prime}$. Abort if there is a tuple $\left(\left(j, i, y_{j}, x_{i}\right), *\right)$ in $L_{1}$. Else, add $\left(\left(j, i, y_{j}, x_{i}\right), d\right)$ into $L_{1}$. Then, let $\ell_{1} \geq 1$ be the index that $\hat{x}_{i}$ appears in $\hat{\mathbf{u}}$, and $\ell_{2} \geq 0$ be the index that $\hat{y}_{j}$ appears in $\mathbf{b}_{0}$, the simulator $\mathcal{S}$ sets $k_{j}=c b_{\ell_{1}, \ell_{2}}$;

$5^{\prime}$. Compute $w_{j}=\mathrm{Cha}\left(k_{j}\right) \in\{0,1\}^{n}$ and return $\left(y_{j}, w_{j}\right)$ to $\mathcal{A}$;

$6^{\prime}$. Compute $\sigma_{j}=\operatorname{Mod}_{2}\left(k_{j}, w_{j}\right)$ and derive the session $\operatorname{key~sk}_{j}=H_{2}\left(i, j, x_{i}, y_{j}, w_{j}, \sigma_{j}\right)$.

Since $\mathbf{v}$ is randomly and independently chosen from $\chi_{\gamma}^{\ell-1}$, the distribution of $c$ is identical to Game $G_{1,6}$ and $G_{1,7}$. Besides, since each $v_{i}$ is invertible in $R_{q}$, we have $\hat{\mathbf{u}}$ is uniformly distributed over $R_{q}^{\ell-1}$, which shows that the distribution of $\hat{x}_{i}$ is identical to Game $G_{1,6}$ and $G_{1,7}$ except with negligible probability. Moreover, if $(\mathbf{u}, \mathbf{B}) \in R_{q}^{\ell} \times R_{q}^{\ell \times \ell}$ is a Ring-LWE challenge tuple in matrix form, we have $\hat{y}_{j}=u_{0} s_{\ell_{2}}+2 e_{0, \ell_{2}}$ and $k_{j}=c b_{\ell_{1}, \ell_{2}}=c u_{\ell_{1}} s_{\ell_{2}}+2 c e_{\ell_{1}, \ell_{2}}=\hat{x}_{i} s_{\ell_{2}}+2 c e_{\ell_{1}, \ell_{2}}=\left(x_{i}+p_{i} c\right) s_{\ell_{2}}+2 c e_{\ell_{1}, \ell_{2}}$ for some randomly chosen $s_{\ell_{2}}, e_{0, \ell_{2}}, e_{\ell_{1}, \ell_{2}} \leftarrow_{r} \chi_{\beta}$. This shows that the view of $\mathcal{A}$ is the same as in Game $G_{1,6}$. While if $(\mathbf{u}, \mathbf{B}) \in R_{q}^{\ell} \times R_{q}^{\ell \times \ell}$ is uniformly distributed over $R_{q}^{\ell} \times R_{q}^{\ell \times \ell}$, we have both $\hat{y}_{j}$ and $k_{j}=c b_{\ell_{1}, \ell_{2}}$ are uniformly distributed over $R_{q}$ (since $c$ is invertible). Thus, the view of $\mathcal{A}$ is the same as in $G_{1,7}$. In all, we have shown that $\mathcal{D}$ can be used to break Ring-LWE assumption if $\mathcal{A}$ can distinguish Game $G_{1,6}$ and $G_{1,7}$.

Claim 9 If $0.97 n>2 \kappa$, we have $\operatorname{Pr}\left[Q_{1,7}\right]=\operatorname{negl}(\kappa)$

Proof. Let $k_{i, \ell}$ be the element "computed" by $\mathcal{S}$ for the $s_{i}^{*}$-th session at party $i^{*}$ in Games $G_{1, \ell}$, and $k_{j, \ell}$ be the element "computed" by $\mathcal{S}$ for the $s_{j}^{*}$-th session at party $j^{*}$. By the correctness of the protocol, we have that $k_{i, 5}=k_{j, 5}+\hat{g}$ for some $\hat{g}$ with small coefficients in $G_{1,5}$. Since we have proven that the view of the adversary before $Q_{1, \ell}$ happens in Game $G_{1,5}, G_{1,6}$ and $G_{1,7}$ is computationally indistinguishable, the equation $k_{i, 7}=k_{j, 7}+\hat{g}^{\prime}$ should still holds for some $\hat{g}^{\prime}$ with small coefficients in the adversary's view until $Q_{1,7}$ happens in $G_{1,7}$. Let $\left(y_{j}, w_{j}\right)$ be output by the $s_{j}^{*}$-th session of party $j=j^{*}$, and $\left(y_{j}, w_{j}^{\prime}\right)$ be

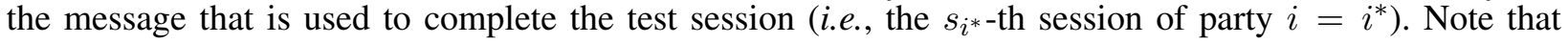
$k_{j, 7}$ is randomly chosen from $R_{q}$, and the adversary can only obtain the information of $k_{j, 7}$ from the public $w_{j}$, the dependence of $\hat{g}$ on $k_{j}$ should be totally determined by the information of $w_{j}$. Thus, we have that $\sigma_{i}^{\prime}=\operatorname{Mod}_{2}\left(k_{i}, w_{j}^{\prime}\right)=\operatorname{Mod}_{2}\left(k_{j}+\hat{g}^{\prime}, w_{j}^{\prime}\right)$ conditioned on $w_{j}$ has high min-entropy by Lemma 4. In other words, the probability that the adversary makes a query $H_{2}\left(i, j, x_{i}, y_{j}, w_{j}^{\prime}, \sigma_{i}^{\prime}\right)$ is at most $2^{-0.97 n}+\operatorname{negl}(\kappa)$, which is negligible in $\kappa$.

Claim $10 \operatorname{Pr}\left[F_{1,7} \mid \neg Q_{1,7}\right]=1 / 2+\operatorname{negl}(\kappa)$

Proof. Let $\left(y_{j}, w_{j}\right)$ be output by the $s_{j}^{*}$-th session of party $j=j^{*},\left(y_{j}, w_{j}^{\prime}\right)$ be the message that is used to complete the test session (i.e., the $s_{i^{*}}$-th session of party $i=i^{*}$ ). We distinguish the following two cases: 
- $w_{j}=w_{j}^{\prime}$ : In this case, we have $\mathrm{sk}_{i}=\mathrm{sk}_{j}=H_{2}\left(i, j, x_{i}, y_{j}, w_{j}, \sigma_{i}\right)$, where $\sigma_{i}=\sigma_{j}=\operatorname{Mod}_{2}\left(k_{j}, w_{j}\right)$. Note that in $G_{1,7}, k_{j}$ is randomly chosen from the uniform distribution over $R_{q}$, we have that $\sigma_{j} \in$ $\{0,1\}^{n}$ (conditioned on $w_{j}$ ) has min-entropy at least $0.97 n$ by Lemma 4 . Thus, the probability that $\mathcal{A}$ has made a $H_{2}$ query with $\sigma_{i}$ is less than $2^{-0.97 n}+\operatorname{negl}(\kappa)$.

- $w_{j} \neq w_{j}^{\prime}$ : By assumption that $Q_{1,7}$ does not happen, we have $\mathcal{A}$ will never make a $H_{2}$ query with $\sigma_{i}$.

In all, the probability that $\mathcal{A}$ has made a $H_{2}$ query with $\sigma_{i}$ is negligible. This claim follows from the fact that if the adversary doesn't make a query with $\sigma_{i}$ exactly, the distribution of $\operatorname{sk}_{i}$ is uniform over $\{0,1\}^{k}$ due to the random oracle property of $H_{2}$, i.e., $\operatorname{Pr}\left[F_{1,7} \mid \neg Q_{1,7}\right]=1 / 2+\operatorname{negl}(\kappa)$.

Combining the claims $1 \sim 10$, we have that Lemma 5 follows.

\subsection{Type II Adversary}

In this subsection, we prove that our AKE is secure against any PPT Type II adversary $\mathcal{A}$.

Lemma 6. Let $n$ be a power of 2 satisfying $0.97 n \geq 2 \kappa$, prime $q>203$ satisfying $q=1 \bmod 2 n$, $\beta=\omega(\alpha \gamma n \sqrt{n \log n})$. Then, if $R L W E_{q, \alpha}$ is hard, the proposed AKE is secure against any PPT Type II adversary $\mathcal{A}$ in the random oracle model.

Proof. We prove this lemma via a sequence of games $G_{2, l}$ for $0 \leq l \leq 6$.

Game $G_{2,0} . \mathcal{S}$ chooses $i^{*}, j^{*} \leftarrow r\{1, \ldots, N\}$ and $s_{i^{*}} \leftarrow r\{1, \ldots, m\}$, and hopes that the adversary will choose $\operatorname{sid}^{*}=\left(\Pi, I, i^{*}, j^{*}, x_{i^{*}},\left(y_{j^{*}}, w_{j^{*}}\right)\right)$ as the test session, where $x_{i^{*}}$ is output by the $s_{i^{*}}$-th session of party $i^{*}$ with intended party $j^{*}$ (note that $\operatorname{sid}^{*}$ has no matching session for Type II adversary). Then, $\mathcal{S}$ chooses $a \leftarrow_{r} R_{q}$, honestly generates static public keys for all parties (by randomly choosing $s_{i}$ and $e_{i}$ from $\chi_{\alpha}$ ), and simulates the attack environment for $\mathcal{A}$. Specifically, $\mathcal{S}$ maintains two tables $L_{1}, L_{2}$ for the random oracles $H_{1}, H_{2}$, respectively, and answers the queries from $\mathcal{A}$ as follows:

- $H_{1}($ in $)$ : If there doesn't exist a tuple $($ in, out $)$ in the $L_{1}$ list, choose an element out $\leftarrow_{r} \chi_{\gamma}$, and add (in, out) to the $L_{1}$ list. Then, return out to $\mathcal{A}$.

- $H_{2}($ in $)$ queries: If there doesn't exist a tuple $($ in, out $)$ in the $L_{2}$ list, choose an element out $\leftarrow_{r}\{0,1\}^{k}$, and add (in, out) to the $L_{2}$ list. Then, return out to $\mathcal{A}$.

- $\operatorname{Send}_{0}(\Pi, I, i, j): \mathcal{A}$ activates a new session of $i$ with intended party $j, \mathcal{S}$ proceeds as follows:

1. Sample $r_{i}, f_{i} \leftarrow_{r} \chi_{\beta}$ and compute $x_{i}=a r_{i}+2 f_{i}$;

2. Compute $c=H_{1}\left(i, j, x_{i}\right), \hat{r}_{i}=s_{i} c+r_{i}$ and $\hat{f}_{i}=e_{i} c+f_{i}$;

3. Letting $\mathbf{z} \in \mathbb{Z}^{2 n}$ be the coefficient vector of $\hat{r}_{i}$ concatenated with the coefficient vector of $\hat{f}_{i}$, and $\mathbf{z}_{1} \in \mathbb{Z}^{2 n}$ be the coefficient vector of $s_{i} c$ concatenated with the coefficient vector of $e_{i} c$, repeat the steps $1 \sim 3$ with probability $1-\min \left(\frac{D_{\mathbb{Z}^{2 n}, \beta}(\mathbf{z})}{M D_{\mathbb{Z}^{2 n}, \beta, \mathbf{z}_{1}}(\mathbf{z})}, 1\right)$;

4. Return $x_{i}$ to $\mathcal{A}$.

- $\operatorname{Send}_{1}\left(\Pi, R, j, i, x_{i}\right): \mathcal{S}$ proceeds as follows:

$1^{\prime}$. Sample $r_{j}, f_{j} \leftarrow_{r} \chi_{\beta}$ and compute $y_{j}=a r_{j}+2 f_{j}$;

$2^{\prime}$. Compute $d=H_{1}\left(j, i, y_{j}, x_{i}\right), \hat{r}_{j}=s_{j} d+r_{j}$ and $\hat{f}_{j}=e_{j} d+f_{j}$;

$3^{\prime}$. Letting $\mathbf{z} \in \mathbb{Z}^{2 n}$ be the coefficient vector of $\hat{r}_{j}$ concatenated with the coefficient vector of $\hat{f}_{j}$, and $\mathbf{z}_{1} \in \mathbb{Z}^{2 n}$ be the coefficient vector of $s_{j} d$ concatenated with the coefficient vector of $e_{j} d$, repeat the steps $1^{\prime} \sim 3^{\prime}$ with probability $1-\min \left(\frac{D_{\mathbb{Z}^{2 n}, \beta}(\mathbf{z})}{M D_{\mathbb{Z}^{2 n}, \beta, \mathbf{z}_{1}}(\mathbf{z})}, 1\right)$; 
$4^{\prime}$. Sample $g_{j} \leftarrow_{r} \chi_{\beta}$ and compute $k_{j}=\left(p_{i} c+x_{i}\right) \hat{r}_{j}+2 c g_{j}$ where $c=H_{1}\left(i, j, x_{i}\right)$;

$5^{\prime}$. Compute $w_{j}=\mathrm{Cha}\left(k_{j}\right) \in\{0,1\}^{n}$ and return $\left(y_{j}, w_{j}\right)$ to $\mathcal{A}$;

$6^{\prime}$. Compute $\sigma_{j}=\operatorname{Mod}_{2}\left(k_{j}, w_{j}\right)$ and derive the session $\operatorname{key~sk}_{j}=H_{2}\left(i, j, x_{i}, y_{j}, w_{j}, \sigma_{j}\right)$.

- $\operatorname{Send}_{2}\left(\Pi, I, i, j, x_{i},\left(y_{j}, w_{j}\right)\right): \mathcal{S}$ computes $k_{i}$ and $\mathrm{sk}_{i}$ as follows:

5. Sample $g_{i} \leftarrow_{r} \chi_{\beta}$ and compute $k_{i}=\left(p_{j} d+y_{j}\right) \hat{r}_{i}+2 d g_{i}$ where $d=H_{1}\left(j, i, y_{j}, x_{i}\right)$;

6. Compute $\sigma_{i}=\operatorname{Mod}_{2}\left(k_{i}, w_{j}\right)$ and derive the session key sk $\mathrm{sk}_{i}=H_{2}\left(i, j, x_{i}, y_{j}, w_{j}, \sigma_{i}\right)$.

- SessionKeyReveal(sid): Let $\operatorname{sid}=(\Pi, *, i, *, *, *, *), \mathcal{S}$ returns $\operatorname{sk}_{i}$ if the session key of sid has been generated.

- Corrupt $(i)$ : Return the static secret key $s_{i}$ of $i$ to $\mathcal{A}$.

- Test(sid): Let sid $=\left(\Pi, I, i, j, x_{i},\left(y_{j}, w_{j}\right)\right)$, if $(i, j) \neq\left(i^{*}, j^{*}\right)$, or $x_{i}$ and $y_{j}$ are not output by the $s_{i^{*}}$-th session of $i^{*}$ and the $s_{j}^{*}$-th session of $j^{*}$, respectively, $\mathcal{S}$ aborts. Otherwise, $\mathcal{S}$ chooses $b \leftarrow_{r}\{0,1\}$ and $s k_{i}^{\prime} \leftarrow_{r}\{0,1\}^{k}$. If $b=0, \mathcal{S}$ returns $s k_{i}^{\prime}$, else it returns the real session $\operatorname{sk}_{i}$ of sid.

Claim 11 The probability that $\mathcal{S}$ will not abort in $G_{2,0}$ is at least $\frac{1}{m N^{2}}$.

Proof. This claim directly follows from the fact that $\mathcal{S}$ randomly chooses $i^{*}, j^{*} \leftarrow r\{1, \ldots, N\}$ and $s_{i^{*}} \leftarrow r$ $\{1, \ldots, m\}$ without $\mathcal{A}$ knowing it.

Game $G_{2,1}$. $\mathcal{S}$ behaves almost the same as in $G_{2,0}$, except in the following cases:

- Send $_{0}(\Pi, I, i, j):$ If $i \neq j^{*}, \mathcal{S}$ answers the query as in Game $G_{2,0}$. Otherwise, it proceeds as follows:

1. Sample an invertible element $c \leftarrow_{r} \chi_{\gamma}$, and $\mathbf{z} \leftarrow_{r} D_{\mathbb{Z}^{2 n}, \beta}$;

2. Interpreting $\mathbf{z}$ as two ring elements $\hat{r}_{i}, \hat{f}_{i} \in R_{q}$, and define $x_{i}=a \hat{r}_{i}+2 \hat{f}_{i}-p_{i} c$.

3. Repeat the steps $1 \sim 3$ with probability $1-1 / M$;

4. Abort if there is a tuple $\left(\left(i, j, x_{i}\right), *\right)$ in $L_{1}$. Else, add $\left(\left(i, j, x_{i}\right), c\right)$ into $L_{1}$. Return $x_{i}$ to $\mathcal{A}$.

- $\operatorname{Send}_{1}\left(\Pi, R, j, i, x_{i}\right)$ : If $j \neq j^{*}, \mathcal{S}$ answers the query as in Game $G_{2,0}$. Otherwise, it proceeds as follows:

$1^{\prime}$. Sample an invertible element $d \leftarrow{ }_{r} \chi_{\gamma}$, and $\mathbf{z} \leftarrow{ }_{r} D_{\mathbb{Z}^{2 n}, \beta}$;

$2^{\prime}$. Interpreting $\mathbf{z}$ as two ring elements $\hat{r}_{j}, \hat{f}_{j} \in R_{q}$, and define $y_{j}=a \hat{r}_{j}+2 \hat{f}_{j}-p_{j} d$.

$3^{\prime}$. Repeat the steps $1^{\prime} \sim 3^{\prime}$ with probability $1-1 / M$;

4'. Abort if there is a tuple $\left(\left(j, i, y_{j}, x_{i}\right), *\right)$ in $L_{1}$. Else, add $\left(\left(j, i, y_{j}, x_{i}\right), d\right)$ into $L_{1}$. Then, sample $g_{j} \leftarrow_{r}$ $\chi_{\beta}$ and compute $k_{j}=\left(p_{i} c+x_{i}\right) \hat{r}_{j}+2 c g_{j}$ where $c=H_{1}\left(i, j, x_{i}\right)$;

$5^{\prime}$. Compute $w_{j}=\mathrm{Cha}\left(k_{j}\right) \in\{0,1\}^{n}$ and return $\left(y_{j}, w_{j}\right)$ to $\mathcal{A}$;

$6^{\prime}$. Compute $\sigma_{j}=\operatorname{Mod}_{2}\left(k_{j}, w_{j}\right)$ and derive the session $\operatorname{key~sk}_{j}=H_{2}\left(i, j, x_{i}, y_{j}, w_{j}, \sigma_{j}\right)$.

In the following, let $F_{2, l}$ denote the event that $\mathcal{A}$ outputs a guess $b^{\prime}$ that equals to $b$ in Game $G_{2, l}$.

Claim 12 If $\beta=\omega(\alpha \gamma n \sqrt{n \log n})$ and $R L W E_{q, \beta}$ is hard, then $\operatorname{Pr}\left[F_{2,1}\right]=\operatorname{Pr}\left[F_{2,0}\right]-\operatorname{negl}(\kappa)$.

Proof. This claim can be proven via a sequence of games similar to that from $G_{1,0}$ to $G_{1,4}$, we omit the details. 
Game $G_{2,2}$. $\mathcal{S}$ behaves almost the same as in $G_{2,1}$, except it replaces the public key for party $j^{*}$ with a uniformly chosen $p_{j^{*}} \leftarrow{ }_{r} R_{q}$.

Claim 13 If $R L W E_{q, \alpha}$ is hard, then $\operatorname{Pr}\left[F_{2,2}\right]=\operatorname{Pr}\left[F_{2,1}\right]-\operatorname{negl}(\kappa)$.

Proof. Since the only difference between $G_{2,1}$ and $G_{2,2}$ is that $\mathcal{S}$ replaces $p_{j^{*}}=a s_{j^{*}}+2 e_{j^{*}}$ with a randomly chosen element in $R_{q}$, an adversary that can distinguish the difference between $G_{2,1}$ and $G_{2,2}$ could be directly used to solve the $\mathrm{RLWE}_{q, \alpha}$ problem.

Game $G_{2,3}$. $\mathcal{S}$ behaves almost the same as in $G_{2,2}$, except in the following cases:

- $\operatorname{Send}_{0}(\Pi, I, i, j)$ : If $(i, j) \neq\left(i^{*}, j^{*}\right)$, or it is not the $s_{i^{*}}$-th session of $i^{*}, \mathcal{S}$ answers the query as in Game $G_{2,2}$. Otherwise, it proceeds as follows:

1. Sample an invertible element $c \leftarrow{ }_{r} \chi_{\gamma}$, and $\mathbf{z} \leftarrow{ }_{r} D_{\mathbb{Z}^{2 n}, \beta}$;

2. Interpreting $\mathbf{z}$ as two ring elements $\hat{r}_{i}, \hat{f}_{i} \in R_{q}$, and define $x_{i}=a \hat{r}_{i}+2 \hat{f}_{i}-p_{i} c$.

3. Repeat the steps $1 \sim 3$ with probability $1-1 / M$;

4. Abort if there is a tuple $\left(\left(i, j, x_{i}\right), *\right)$ in $L_{1}$. Else, add $\left(\left(i, j, x_{i}\right), c\right)$ into $L_{1}$. Return $x_{i}$ to $\mathcal{A}$.

Claim 14 If $\beta=\omega(\alpha \gamma n \sqrt{n \log n})$ and $R L W E_{q, \beta}$ is hard, then $\operatorname{Pr}\left[F_{2,3}\right]=\operatorname{Pr}\left[F_{2,2}\right]-\operatorname{negl}(\kappa)$.

Proof. This claim can be proven via a sequence of games similar to that from $G_{1,2}$ to $G_{1,4}$, we omit the details.

Game $G_{2,4}$. $\mathcal{S}$ first randomly chooses $u \leftarrow_{r} R_{q}$, then computes $\mathbf{v}_{0}=\left(v_{0,1}, \ldots, v_{0, \ell-1}\right) \in R_{q}^{\ell}$ and $\mathbf{v}_{1}=$ $\left(v_{1,1}, \ldots, v_{1, \ell-1}\right) \in R_{q}^{\ell}$ where $v_{0, \ell^{\prime}}=a \hat{r}_{\ell^{\prime}}+2 \hat{f}_{\ell^{\prime}}, v_{1, \ell^{\prime}}=u \hat{r}_{\ell^{\prime}}+2 g_{\ell^{\prime}}$ for $\hat{r}_{\ell^{\prime}}, \hat{f}_{\ell_{1}}, g_{\ell^{\prime}} \leftarrow \chi_{\beta}$. Then, it sets $p_{j^{*}}=u$, and behaves almost the same as in $G_{2,3}$, except in the following cases:

- $\operatorname{Send}_{0}(\Pi, I, i, j)$ : If $(i, j) \neq\left(i^{*}, j^{*}\right)$, or it is not the $s_{i^{*}}$-th session of $i^{*}, \mathcal{S}$ answers the query as in Game $G_{2,3}$. Otherwise, it proceeds as follows:

1. Sample an invertible element $c \leftarrow_{r} \chi_{\gamma}$, and set $\hat{x}_{i}$ be the first unused element in $\mathbf{v}_{0}$;

2. Define $x_{i}=\hat{x}_{i}-p_{i} c$.

3. Repeat the steps $1 \sim 3$ with probability $1-1 / M$;

4. Abort if there is a tuple $\left(\left(i, j, x_{i}\right), *\right)$ in $L_{1}$. Else, add $\left(\left(i, j, x_{i}\right), c\right)$ into $L_{1}$. Return $x_{i}$ to $\mathcal{A}$.

- $\operatorname{Send}_{2}\left(\Pi, I, i, j, x_{i},\left(y_{j}, w_{j}\right)\right)$ : If $(i, j) \neq\left(i^{*}, j^{*}\right)$, or it is not the $s_{i^{*}}$-th session of $i^{*}, \mathcal{S}$ answers the query as in Game $G_{2,3}$. Otherwise, it proceeds as follows::

5. Let $\ell^{*}$ be the index that $\hat{x}_{i}$ appears in $\mathbf{v}_{0}$, namely, $\hat{x}_{i}=a \hat{r}_{\ell^{*}}+2 \hat{f}_{\ell^{*}}$, the simulator $\mathcal{S}$ sets $d=H_{1}(j, i$, $\left.y_{j}, x_{i}\right)$, and computes $k_{i}=d v_{1, \ell^{*}}+y_{j} \hat{r}_{\ell^{*}}=d\left(u \hat{r}_{\ell^{*}}+2 g_{\ell^{*}}\right)+y_{j} \hat{r}_{\ell^{*}}=\left(p_{j} d+y_{j}\right) \hat{r}_{\ell^{*}}+2 d g_{\ell^{*}}$;

6. Compute $\sigma_{i}=\operatorname{Mod}_{2}\left(k_{i}, w_{j}\right)$ and derive the session key $\operatorname{sk}_{i}=H_{2}\left(i, j, x_{i}, y_{j}, w_{j}, \sigma_{i}\right)$.

Claim 15 Game $G_{2,4}$ is identical to Game $G_{2,3}$. In particular, we have $\operatorname{Pr}\left[F_{2,4}\right]=\operatorname{Pr}\left[F_{2,3}\right]$.

Proof. This claim simply from the fact that Game $G_{2,4}$ is just a conceptual change of Game $G_{2,3}$. 
Game $G_{2,5}$. $\mathcal{S}$ chooses $u_{0}, u_{1} \leftarrow_{r} R_{q}$, and $\mathbf{v}_{0}=\left(v_{0,1}, \ldots, v_{0, \ell-1}\right), \mathbf{v}_{1}=\left(v_{1,1}, \ldots, v_{1, \ell-1}\right) \leftarrow_{r} R_{q}^{\ell}$. Then, it sets $a=u_{0}$ and $p_{j^{*}}=u_{1}$, and behaves almost the same as in $G_{2,4}$, except in the following case:

- $\operatorname{Send}_{2}\left(\Pi, I, i, j, x_{i},\left(y_{j}, w_{j}\right)\right)$ : If $(i, j) \neq\left(i^{*}, j^{*}\right)$, or it is not the $s_{i^{*}}$-th session of $i^{*}, \mathcal{S}$ answers the query as in Game $G_{2,3}$. Otherwise, it proceeds as follows:

5. Randomly choose $k_{i} \leftarrow_{r} R_{q}$;

6. Compute $\sigma_{i}=\operatorname{Mod}_{2}\left(k_{i}, w_{j}\right)$ and derive the session key sk $\mathrm{sk}_{i}=H_{2}\left(i, j, x_{i}, y_{j}, w_{j}, \sigma_{i}\right)$.

Claim 16 Let $n$ be a power of 2 satisfying $0.97 n \geq 2 \kappa$, prime $q>203$ satisfying $q=1 \bmod 2 n$, $\beta=$ $\omega(\alpha \gamma n \sqrt{n \log n})$. Then, if $R L W E_{q, \beta}$ is hard, Game $G_{2,5}$ is computationally indistinguishable from $G_{2,4}$. In particular, $\operatorname{Pr}\left[F_{2,4}\right]=\operatorname{Pr}\left[F_{2,5}\right]+\operatorname{negl}(\kappa)=1 / 2+\operatorname{negl}(\kappa)$.

Proof. Note that before generating $k_{i}$, the only difference between $G_{2,4}$ and $G_{2,5}$ is that $\mathcal{S}$ replaces the RingLWE tuple $\left(\left(u_{0}, u_{1}\right),\left(\mathbf{v}_{0}, \mathbf{v}_{1}\right)\right) \in R_{q}^{2} \times R_{q}^{2 \times \ell}$ with randomly chosen elements in $R_{q}^{2} \times R_{q}^{2 \times \ell}$. In other words, under the Ring-LWE assumption, we have that the adversary's views before generating $k_{i}$ in both $G_{2,4}$ and $G_{2,5}$ are computationally indistinguishable. Besides, since $H_{2}$ is a random oracle, no matter whether $\mathcal{A}$ obtains $\mathrm{sk}_{i}$ or not, the value of $\mathrm{sk}_{i}$ will not affect the view of the adversary, especially about the knowledge of $k_{i}$.

Now, let $k_{i}=d v_{1, \ell^{*}}+y_{j} r_{\ell^{*}}$ be the element computed by using $d=H_{1}\left(j^{*}, i^{*}, y_{j}, x_{i}\right)$ in Game $G_{2,4}$ with non-negligible probability $\delta$. Fixing $\mathbf{v}_{0}$ and $\mathbf{v}_{1}$ (note that all those values are chosen by $\mathcal{S}$, and are independent from the adversary's behaviors), $\mathcal{S}$ reprograms the hash query $H_{1}\left(j^{*}, i^{*}, y_{j}, x_{i}\right)=\tilde{d} \neq d$ by using another randomly chosen $\tilde{d} \leftarrow_{r} \chi_{\gamma}$ (recall that $y_{j}$ is not generated by the simulator $\mathcal{S}$, such a $H_{1}$ query is made by the adversary $\mathcal{A}$ ). According to the forking lemma [6], $\mathcal{A}$ will use the same $y_{j}$ to complete the test session with probability at least $\delta\left(\delta / q_{h}-2^{-n}\right)$, where $q_{h}$ is maximum number of $H_{1}$ queries. In such a case, we have $\tilde{k}_{i}=\tilde{d} v_{1, \ell^{*}}+y_{j} \hat{r}_{\ell^{*}}$ and $\tilde{k}_{i}=k_{i}+(\tilde{d}-d) v_{1, \ell^{*}}$.

Denote $\hat{k}_{i}^{\prime}$ and $k_{i}^{\prime}$ (i.e., the values determined before and after $\mathcal{S}$ reprograms the $H_{1}$ query) as the target values in the $\mathcal{A}$ 's view in Game $G_{2,5}$, we have that $\tilde{k}_{i}^{\prime}=k_{i}^{\prime}+(\tilde{d}-d) v_{1, \ell^{*}}$ should still hold in the adversary's view. Considering the fact that $v_{1, \ell^{*}}$ is uniformly and randomly chosen from $R_{q}$ and $(\tilde{d}-d)$ is invertible with overwhelming probability for appropriate choice of $\gamma$ and $q$ by Lemma 10 in [65], we have that the adversary essentially has no knowledge of $\tilde{k}_{i}^{\prime}$ and $k_{i}^{\prime}$. Therefore, the choice of $k_{j} \leftarrow_{r} R_{q}$ in Game $G_{2,5}$ is correctly distributed in the adversary's view, and thus will not help the adversary $\mathcal{A}$ to distinguish $G_{2,5}$ from $G_{2,4}$. In other words, we have that $\operatorname{Pr}\left[F_{2,4}\right]=\operatorname{Pr}\left[F_{2,5}\right]+\operatorname{negl}(\kappa)$.

Finally, since $k_{i}$ is randomly and uniformly chosen from $R_{q}$ in Game $G_{2,5}$, and is independent from $\mathcal{A}$ 's view, we have $\sigma_{i}=\operatorname{Mod}_{2}\left(k_{i}, w_{j}\right) \in\{0,1\}^{n}$ has very high min-entropy by Lemma 4 . In other words, the probability that the adversary makes a query $H_{2}\left(i, j, x_{i}, y_{j}, w_{j}, \sigma_{i}\right)$ is at most $2^{-0.97 n}+\operatorname{negl}(\kappa)$, which is negligible in $\kappa$. Since $H_{2}$ is a random oracle, we have $\operatorname{Pr}\left[F_{2,5}\right]=1 / 2+\operatorname{negl}(\kappa)$, which completes the proof.

\subsection{Type III Adversary}

In this subsection, we prove that our AKE is secure against any PPT Type III adversary $\mathcal{A}$.

Lemma 7. Let $n$ be a power of 2 satisfying $0.97 n \geq 2 \kappa$, prime $q>203$ satisfying $q=1 \bmod 2 n$, $\beta=\omega(\alpha \gamma n \sqrt{n \log n})$. Then, if RLWE $E_{q, \alpha}$ is hard, the proposed AKE is secure against any PPT Type III adversary $\mathcal{A}$ in the random oracle model.

Proof. We prove this lemma via a sequence of games $G_{3, l}$ for $0 \leq l \leq 6$. 
Game $G_{3,0}$. $\mathcal{S}$ chooses $i^{*}, j^{*} \leftarrow_{r}\{1, \ldots, N\}$ and $s_{j^{*}} \leftarrow_{r}\{1, \ldots, m\}$, and hopes that the adversary will choose $\operatorname{sid}^{*}=\left(\Pi, R, j^{*}, i^{*}, x_{i^{*}},\left(y_{j^{*}}, w_{j^{*}}\right)\right)$ as the test session, where $\left(y_{j^{*}}, w_{j^{*}}\right)$ is output by the $s_{j}^{*}$-th session of party $j^{*}$ activated by a $\operatorname{Send}_{0}\left(\Pi, R, j^{*}, i^{*}, x_{i^{*}}\right)$ for some $x_{i^{*}}$. Then, $\mathcal{S}$ chooses $a \leftarrow_{r} R_{q}$, honestly generates static public keys for all parties (by randomly choosing $s_{i}$ and $e_{i}$ from $\chi_{\alpha}$ ), and simulates the attack environment for $\mathcal{A}$. Specifically, $\mathcal{S}$ maintains two tables $L_{1}, L_{2}$ for the random oracles $H_{1}, H_{2}$, respectively, and answers the queries from $\mathcal{A}$ as follows:

- $H_{1}($ in $)$ : If there doesn't exist a tuple $($ in,$o u t)$ in the $L_{1}$ list, choose an element out $\leftarrow_{r} \chi_{\gamma}$, and add (in, out) to the $L_{1}$ list. Then, return out to $\mathcal{A}$.

- $H_{2}($ in $)$ queries: If there doesn't exist a tuple (in, out) in the $L_{2}$ list, choose an element out $\leftarrow_{r}\{0,1\}^{k}$, and add (in, out) to the $L_{2}$ list. Then, return out to $\mathcal{A}$.

- $\operatorname{Send}_{0}(\Pi, I, i, j): \mathcal{A}$ activates a new session of $i$ with intended party $j, \mathcal{S}$ proceeds as follows:

1. Sample $r_{i}, f_{i} \leftarrow_{r} \chi_{\beta}$ and compute $x_{i}=a r_{i}+2 f_{i}$;

2. Compute $c=H_{1}\left(i, j, x_{i}\right), \hat{r}_{i}=s_{i} c+r_{i}$ and $\hat{f}_{i}=e_{i} c+f_{i}$;

3. Letting $\mathbf{z} \in \mathbb{Z}^{2 n}$ be the coefficient vector of $\hat{r}_{i}$ concatenated with the coefficient vector of $\hat{f}_{i}$, and $\mathbf{z}_{1} \in \mathbb{Z}^{2 n}$ be the coefficient vector of $s_{i} c$ concatenated with the coefficient vector of $e_{i} c$, repeat the steps $1 \sim 3$ with probability $1-\min \left(\frac{D_{\mathbb{Z}^{2 n}, \beta}(\mathbf{z})}{M D_{\mathbb{Z}^{2 n}, \beta, \mathbf{z}_{1}}(\mathbf{z})}, 1\right)$.

4. Return $x_{i}$ to $\mathcal{A}$.

- $\operatorname{Send}_{1}\left(\Pi, R, j, i, x_{i}\right): \mathcal{S}$ proceeds as follows:

$1^{\prime}$. Sample $r_{j}, f_{j} \leftarrow_{r} \chi_{\beta}$ and compute $y_{j}=a r_{j}+2 f_{j}$;

$2^{\prime}$. Compute $d=H_{1}\left(j, i, y_{j}, x_{i}\right), \hat{r}_{j}=s_{j} d+r_{j}$ and $\hat{f}_{j}=e_{j} d+f_{j}$;

$3^{\prime}$. Letting $\mathbf{z} \in \mathbb{Z}^{2 n}$ be the coefficient vector of $\hat{r}_{j}$ concatenated with the coefficient vector of $\hat{f}_{j}$, and $\mathbf{z}_{1} \in \mathbb{Z}^{2 n}$ be the coefficient vector of $s_{j} d$ concatenated with the coefficient vector of $e_{j} d$, repeat the steps $1^{\prime} \sim 3^{\prime}$ with probability $1-\min \left(\frac{D_{\mathbb{Z}^{2 n}, \beta}(\mathbf{z})}{M D_{\mathbb{Z}^{2 n}, \beta, \mathbf{z}_{1}}(\mathbf{z})}, 1\right)$;

$4^{\prime}$. Sample $g_{j} \leftarrow_{r} \chi_{\beta}$ and compute $k_{j}=\left(p_{i} c+x_{i}\right) \hat{r}_{j}+2 c g_{j}$ where $c=H_{1}\left(i, j, x_{i}\right)$;

$5^{\prime}$. Compute $w_{j}=\mathrm{Cha}\left(k_{j}\right) \in\{0,1\}^{n}$ and return $\left(y_{j}, w_{j}\right)$ to $\mathcal{A}$;

$6^{\prime}$. Compute $\sigma_{j}=\operatorname{Mod}_{2}\left(k_{j}, w_{j}\right)$ and derive the session key sk $\mathrm{sk}_{j}=H_{2}\left(i, j, x_{i}, y_{j}, w_{j}, \sigma_{j}\right)$.

- $\operatorname{Send}_{2}\left(\Pi, I, i, j, x_{i},\left(y_{j}, w_{j}\right)\right): \mathcal{S}$ computes $k_{i}$ and $\mathrm{sk}_{i}$ as follows:

5. Sample $g_{i} \leftarrow_{r} \chi_{\beta}$ and compute $k_{i}=\left(p_{j} d+y_{j}\right) \hat{r}_{i}+2 d g_{i}$ where $d=H_{1}\left(j, i, y_{j}, x_{i}\right)$;

6. Compute $\sigma_{i}=\operatorname{Mod}_{2}\left(k_{i}, w_{j}\right)$ and derive the session key sk $\operatorname{si}_{i}=H_{2}\left(i, j, x_{i}, y_{j}, w_{j}, \sigma_{i}\right)$.

- SessionKeyReveal(sid): Let $\operatorname{sid}=(\Pi, *, i, *, *, *, *), \mathcal{S}$ returns $\operatorname{sk}_{i}$ if the session key of sid has been generated.

- Corrupt $(i)$ : Return the static secret key $s_{i}$ of $i$ to $\mathcal{A}$.

- Test(sid): Let sid $=\left(\Pi, I, i, j, x_{i},\left(y_{j}, w_{j}\right)\right)$, if $(i, j) \neq\left(i^{*}, j^{*}\right)$, or $x_{i}$ and $y_{j}$ are not output by the $s_{i^{*}}$-th session of $i^{*}$ and the $s_{j}^{*}$-th session of $j^{*}$, respectively, $\mathcal{S}$ aborts. Otherwise, $\mathcal{S}$ chooses $b \leftarrow_{r}\{0,1\}$ and $s k_{i}^{\prime} \leftarrow_{r}\{0,1\}^{k}$. If $b=0, \mathcal{S}$ returns $s k_{i}^{\prime}$, else it returns the real session $\operatorname{sk}_{i}$ of sid.

Claim 17 The probability that $\mathcal{S}$ will not abort in $G_{3,0}$ with probability at least $\frac{1}{m N^{2}}$.

Proof. This claim directly follows from the fact that $\mathcal{S}$ randomly chooses $i^{*}, j^{*} \leftarrow r\{1, \ldots, N\}$ and $s_{j}^{*} \leftarrow r$ $\{1, \ldots, m\}$ independently from the view of $\mathcal{A}$. 
Game $G_{3,1}$. $\mathcal{S}$ behaves almost the same as in $G_{3,0}$, except in the following cases:

- Send $(\Pi, I, i, j):$ If $i \neq i^{*}, \mathcal{S}$ answers the query as in Game $G_{2,0}$. Otherwise, it proceeds as follows:

1. Sample an invertible element $c \leftarrow_{r} \chi_{\gamma}$, and $\mathbf{z} \leftarrow{ }_{r} D_{\mathbb{Z}^{2 n}, \beta}$;

2. Interpreting $\mathbf{z}$ as two ring elements $\hat{r}_{i}, \hat{f}_{i} \in R_{q}$, and define $x_{i}=a \hat{r}_{i}+2 \hat{f}_{i}-p_{i} c$.

3. Repeat the steps $1 \sim 3$ with probability $1-1 / M$;

4. Abort if there is a tuple $\left(\left(i, j, x_{i}\right), *\right)$ in $L_{1}$. Else, add $\left(\left(i, j, x_{i}\right), c\right)$ into $L_{1}$. Return $x_{i}$ to $\mathcal{A}$.

- Send ${ }_{1}\left(\Pi, R, j, i, x_{i}\right)$ : If $j \neq i^{*}, \mathcal{S}$ answers the query as in Game $G_{2,0}$. Otherwise, it proceeds as follows:

$1^{\prime}$. Sample an invertible element $d \leftarrow_{r} \chi_{\gamma}$, and $\mathbf{z} \leftarrow_{r} D_{\mathbb{Z}^{2 n}, \beta}$;

$2^{\prime}$. Interpreting $\mathbf{z}$ as two ring elements $\hat{r}_{j}, \hat{f}_{j} \in R_{q}$, and define $y_{j}=a \hat{r}_{j}+2 \hat{f}_{j}-p_{j} d$.

$3^{\prime}$. Repeat the steps $1^{\prime} \sim 3^{\prime}$ with probability $1-1 / M$;

$4^{\prime}$. Abort if there is a tuple $\left(\left(j, i, y_{j}, x_{i}\right), *\right)$ in $L_{1}$. Else, add $\left(\left(j, i, y_{j}, x_{i}\right), d\right)$ into $L_{1}$. Then, sample $g_{j} \leftarrow_{r}$ $\chi_{\beta}$ and compute $k_{j}=\left(p_{i} c+x_{i}\right) \hat{r}_{j}+2 c g_{j}$ where $c=H_{1}\left(i, j, x_{i}\right)$;

$5^{\prime}$. Compute $w_{j}=\operatorname{Cha}\left(k_{j}\right) \in\{0,1\}^{n}$ and return $\left(y_{j}, w_{j}\right)$ to $\mathcal{A}$;

$6^{\prime}$. Compute $\sigma_{j}=\operatorname{Mod}_{2}\left(k_{j}, w_{j}\right)$ and derive the session key sk $\operatorname{sk}_{j}=H_{2}\left(i, j, x_{i}, y_{j}, w_{j}, \sigma_{j}\right)$.

In the following, we use $F_{3, l}$ to denote the event that $\mathcal{A}$ outputs a guess $b^{\prime}$ that equals to $b$ in Game $G_{3, l}$.

Claim 18 If $\beta=\omega(\alpha \gamma n \sqrt{n \log n})$ and $R L W E_{q, \beta}$ is hard, then $\operatorname{Pr}\left[F_{3,1}\right]=\operatorname{Pr}\left[F_{3,0}\right]-\operatorname{negl}(\kappa)$.

Proof. This claim can be proven via a sequence of games similar to that from $G_{1,0}$ to $G_{1,4}$, we omit the details.

Game $G_{3,2}$. $\mathcal{S}$ behaves almost the same as in $G_{3,1}$, except it replaces the public key for party $i^{*}$ with a randomly chosen $p_{i^{*}} \leftarrow{ }_{r} R_{q}$.

Claim 19 If $R L W E_{q, \alpha}$ is hard, then $\operatorname{Pr}\left[F_{3,2}\right]=\operatorname{Pr}\left[F_{3,1}\right]-\operatorname{negl}(\kappa)$.

Proof. Since the only difference between $G_{3,1}$ and $G_{3,2}$ is that $\mathcal{S}$ replaces $p_{i^{*}}=a s_{i^{*}}+2 e_{i^{*}}$ with a randomly chosen element in $R_{q}$, an adversary that can distinguish the difference between $G_{3,1}$ and $G_{3,2}$ could be directly used to solve the $\mathrm{RLWE}_{q, \alpha}$ problem.

Game $G_{3,3}$. $\mathcal{S}$ behaves almost the same as in $G_{3,2}$, except in the following case:

- $\operatorname{Send}_{1}\left(\Pi, R, j, i, x_{i}\right):$ If $(i, j) \neq\left(i^{*}, j^{*}\right)$, or it is not the $s_{j}^{*}$-th session of $j^{*}, \mathcal{S}$ answers the query as in Game $G_{3,2}$. Otherwise, it proceeds as follows:

$1^{\prime}$. Sample an invertible element $d \leftarrow_{r} \chi_{\gamma}$, and $\mathbf{z} \leftarrow_{r} D_{\mathbb{Z}^{2 n}, \beta}$;

$2^{\prime}$. Interpreting $\mathbf{z}$ as two ring elements $\hat{r}_{j}, \hat{f}_{j} \in R_{q}$, and define $y_{j}=a \hat{r}_{j}+2 \hat{f}_{j}-p_{j} d$.

$3^{\prime}$. Repeat the steps $1^{\prime} \sim 3^{\prime}$ with probability $1-1 / M$;

$4^{\prime}$. Abort if there is a tuple $\left(\left(j, i, y_{j}, x_{i}\right), *\right)$ in $L_{1}$. Else, add $\left(\left(j, i, y_{j}, x_{i}\right), d\right)$ into $L_{1}$. Then, sample $g_{j} \leftarrow_{r}$ $\chi_{\beta}$ and compute $k_{j}=\left(p_{i} c+x_{i}\right) \hat{r}_{j}+2 c g_{j}$ where $c=H_{1}\left(i, j, x_{i}\right)$;

$5^{\prime}$. Compute $w_{j}=\operatorname{Cha}\left(k_{j}\right) \in\{0,1\}^{n}$ and return $\left(y_{j}, w_{j}\right)$ to $\mathcal{A}$;

$6^{\prime}$. Compute $\sigma_{j}=\operatorname{Mod}_{2}\left(k_{j}, w_{j}\right)$ and derive the session key sk $\mathrm{sk}_{j}=H_{2}\left(i, j, x_{i}, y_{j}, w_{j}, \sigma_{j}\right)$.

Claim 20 If $\beta=\omega(\alpha \gamma n \sqrt{n \log n})$ and $R L W E_{q, \beta}$ is hard, then $\operatorname{Pr}\left[F_{3,3}\right]=\operatorname{Pr}\left[F_{3,2}\right]-\operatorname{negl}(\kappa)$.

Proof. This claim can be proven via a sequence of games similar to that from $G_{1,0}$ to $G_{1,2}$, we omit the details. 
Game $G_{3,4}$. $\mathcal{S}$ first randomly chooses $u \leftarrow{ }_{r} R_{q}$, then computes $\mathbf{v}_{0}=\left(v_{0,1}, \ldots, v_{0, \ell-1}\right) \in R_{q}^{\ell}$ and $\mathbf{v}_{1}=$ $\left(v_{1,1}, \ldots, v_{1, \ell-1}\right) \in R_{q}^{\ell}$ where $v_{0, \ell^{\prime}}=a \hat{r}_{\ell^{\prime}}+2 \hat{f}_{\ell^{\prime}}, v_{1, \ell^{\prime}}=u \hat{r}_{\ell^{\prime}}+2 g_{\ell^{\prime}}$ for $\hat{r}_{\ell^{\prime}}, \hat{f}_{\ell_{1}}, g_{\ell^{\prime}} \leftarrow \chi_{\beta}$. Then, it sets $p_{i^{*}}=u$, and behaves almost the same as in $G_{3,3}$, except in the following case:

- $\operatorname{Send}_{1}\left(\Pi, R, j, i, x_{i}\right)$ : If $(i, j) \neq\left(i^{*}, j^{*}\right)$, or it is not the $s_{j}^{*}$-th session of $j^{*}, \mathcal{S}$ answers the query as in Game $G_{3,2}$. Otherwise, it proceeds as follows:

$1^{\prime}$. Sample an invertible element $d \leftarrow_{r} \chi_{\gamma}$, and set $\hat{y}_{j}$ be the first unused element in $\mathbf{v}_{0}$;

$2^{\prime}$. Define $y_{j}=\hat{y}_{j}-p_{j} d$;

$3^{\prime}$. Repeat the steps $1^{\prime} \sim 3^{\prime}$ with probability $1-1 / M$;

$4^{\prime}$. Abort if there is a tuple $\left(\left(j, i, y_{j}, x_{i}\right), *\right)$ in $L_{1}$. Else, add $\left(\left(j, i, y_{j}, x_{i}\right), d\right)$ into $L_{1}$. Then, the simulator $\mathcal{S}$ sets $k_{j}=c v_{1, \ell^{*}}+x_{i} \hat{r}_{\ell^{*}}=c\left(u \hat{r}_{\ell^{*}}+2 g_{\ell^{*}}\right)+x_{i} \hat{r}_{\ell^{*}}=\left(p_{i} c+x_{i}\right) \hat{r}_{\ell^{*}}+2 c g_{\ell^{*}}$, where $\ell^{*}$ is the index that $\hat{y}_{j}$ appears in $\mathbf{v}_{0}$ (i.e., $\left.\hat{y}_{j}=a \hat{r}_{\ell^{*}}+2 \hat{f}_{\ell^{*}}\right)$ and $c=H_{1}\left(i, j, x_{i}\right)$;

$5^{\prime}$. Compute $w_{j}=\mathrm{Cha}\left(k_{j}\right) \in\{0,1\}^{n}$ and return $\left(y_{j}, w_{j}\right)$ to $\mathcal{A}$;

$6^{\prime}$. Compute $\sigma_{j}=\operatorname{Mod}_{2}\left(k_{j}, w_{j}\right)$ and derive the session $\operatorname{key~sk}_{j}=H_{2}\left(i, j, x_{i}, y_{j}, w_{j}, \sigma_{j}\right)$.

Claim 21 Game $G_{3,4}$ is identical to Game $G_{3,3}$. In particular, we have $\operatorname{Pr}\left[F_{3,4}\right]=\operatorname{Pr}\left[F_{3,3}\right]$.

Proof. This claim simply from the fact that Game $G_{3,4}$ is just a conceptual change of Game $G_{3,3}$.

Game $G_{3,5}$. $\mathcal{S}$ chooses $u_{0}, u_{1} \leftarrow{ }_{r} R_{q}$, and $\mathbf{v}_{0}=\left(v_{0,1}, \ldots, v_{0, \ell-1}\right), \mathbf{v}_{1}=\left(v_{1,1}, \ldots, v_{1, \ell-1}\right) \leftarrow_{r} R_{q}^{\ell}$. Then, it sets $a=u_{0}$ and $p_{j^{*}}=u_{1}$, and behaves almost the same as in $G_{3,4}$ except the following cases:

- $\operatorname{Send}_{1}\left(\Pi, R, j, i, x_{i}\right):$ If $(i, j) \neq\left(i^{*}, j^{*}\right)$, or it is not the $s_{j}^{*}$-th session of $j^{*}, \mathcal{S}$ answers the query as in Game $G_{3,2}$. Otherwise, it proceeds as follows:

$1^{\prime}$. Sample an invertible element $d \leftarrow_{r} \chi_{\gamma}$, and set $\hat{y}_{j}$ be the first unused element in $\mathbf{v}_{0}$;

$2^{\prime}$. Define $y_{j}=\hat{y}_{j}-p_{j} d$;

$3^{\prime}$. Repeat the steps $1^{\prime} \sim 3^{\prime}$ with probability $1-1 / M$;

$4^{\prime}$. Abort if there is a tuple $\left(\left(j, i, y_{j}, x_{i}\right), *\right)$ in $L_{1}$. Else, add $\left(\left(j, i, y_{j}, x_{i}\right), d\right)$ into $L_{1}$. Then, $\mathcal{S}$ randomly chooses $k_{j} \leftarrow_{r} R_{q}$;

$5^{\prime}$. Compute $w_{j}=\mathrm{Cha}\left(k_{j}\right) \in\{0,1\}^{n}$ and return $\left(y_{j}, w_{j}\right)$ to $\mathcal{A}$;

$6^{\prime}$. Compute $\sigma_{j}=\operatorname{Mod}_{2}\left(k_{j}, w_{j}\right)$ and derive the session $\operatorname{key~sk}_{j}=H_{2}\left(i, j, x_{i}, y_{j}, w_{j}, \sigma_{j}\right)$.

Claim 22 Let $n$ be a power of 2 satisfying $0.97 n \geq 2 \kappa$, prime $q>203$ satisfying $q=1 \bmod 2 n$, $\beta=$ $\omega(\alpha \gamma n \sqrt{n \log n})$. Then, if $R L W E_{q, \beta}$ is hard, Game $G_{3,5}$ is computationally indistinguishable from $G_{3,4}$. In particular, $\operatorname{Pr}\left[F_{3,4}\right]=\operatorname{Pr}\left[F_{3,5}\right]+\operatorname{negl}(\kappa)=1 / 2+\operatorname{negl}(\kappa)$.

Proof. Note that before generating $k_{j}$, the only difference between $G_{3,4}$ and $G_{3,5}$ is that $\mathcal{S}$ replaces the RingLWE tuple $\left(\left(u_{0}, u_{1}\right),\left(\mathbf{v}_{0}, \mathbf{v}_{1}\right)\right) \in R_{q}^{2} \times R_{q}^{2 \times \ell}$ with randomly chosen elements in $R_{q}^{2} \times R_{q}^{2 \times \ell}$. In other words, under the Ring-LWE assumption, we have that the adversary's views before generating $k_{j}$ in both $G_{3,4}$ and $G_{3,5}$ are computationally indistinguishable. Besides, since $H_{2}$ is a random oracle, no matter whether $\mathcal{A}$ obtains $\mathrm{sk}_{j}$ or not, the value of $\mathrm{sk}_{j}$ will not affect the view of the adversary, especially about the knowledge of $k_{j}$.

Now, let $k_{j}=c v_{1, \ell^{*}}+x_{i} r_{\ell^{*}}$ be the element computed by using $c=H_{1}\left(i^{*}, j^{*}, x_{i}\right)$ in Game $G_{3,4}$ with non-negligible probability $\delta$. Fixing $\mathbf{v}_{0}$ and $\mathbf{v}_{1}$ (note that all those values are chosen by $\mathcal{S}$, and are independent from the adversary's behaviors), $\mathcal{S}$ reprograms the hash query $H_{1}\left(i^{*}, j^{*}, x_{i}\right)=\tilde{c} \neq c$ by using 
another randomly chosen $\tilde{c} \leftarrow_{r} \chi_{\gamma}$ (recall that $x_{i}$ is not generated by the simulator $\mathcal{S}$, such a $H_{1}$ query is made by the adversary $\mathcal{A}$ ). According to the forking lemma [6], $\mathcal{A}$ will use the same $x_{i}$ to complete the test session with probability at least $\delta\left(\delta / q_{h}-2^{-n}\right)$, where $q_{h}$ is maximum number of $H_{1}$ queries. In such a case, we have $\tilde{k}_{j}=\tilde{c} v_{1, \ell^{*}}+x_{i} \hat{r}_{\ell^{*}}$ and $\tilde{k}_{j}=k_{j}+(\tilde{c}-c) v_{1, \ell^{*}}$.

Denote $\tilde{k}_{j}^{\prime}$ and $k_{j}^{\prime}$ (i.e., the values determined before and after $\mathcal{S}$ reprograms the $H_{1}$ query) as the target values in the $\mathcal{A}$ 's view in Game $G_{3,5}$, we have that $\tilde{k}_{j}^{\prime}=k_{j}^{\prime}+(\tilde{c}-c) v_{1, \ell^{*}}$ should still hold in the adversary's view before seeing corresponding $w_{j}$ 's. Considering the fact that $v_{1, \ell^{*}}$ is uniformly and randomly chosen from $R_{q}$ and $(\tilde{c}-c)$ is invertible with overwhelming probability for appropriate choice of $\gamma$ and $q$ by Lemma 10 in [65], we have that the adversary essentially has no knowledge of $\tilde{k}_{j}^{\prime}$ and $k_{j}^{\prime}$ before seeing $w_{j}$ 's. In other words, before seeing $w_{j}$, the distribution of $k_{j}$ in the adversary's view should be uniformly distributed over $R_{q}$. Therefore, the choice of $k_{j} \leftarrow_{r} R_{q}$ in Game $G_{3,5}$ is correctly distributed in the adversary's view, and thus the adversary should not distinguish the difference between $G_{3,5}$ and $G_{3,4}$ without the knowledge of $w_{j}$ 's. Note that the $w_{j}$ 's in both $G_{3,5}$ and $G_{3,4}$ are computed from $k_{j}$ following the protocol, the knowledge of $w_{j}$ will not help the adversary $\mathcal{A}$ to distinguish $G_{3,5}$ from $G_{3,4}$. In other words, we have that $\operatorname{Pr}\left[F_{3,4}\right]=$ $\operatorname{Pr}\left[F_{3,5}\right]+\operatorname{negl}(\kappa)$.

Finally, since $k_{j}$ is randomly and uniformly chosen from $R_{q}$ in Game $G_{3,5}$, and is independent from $\mathcal{A}$ 's view, we have $\sigma_{j}=\operatorname{Mod}_{2}\left(k_{j}, w_{j}\right) \in\{0,1\}^{n}$ has very high min-entropy by Lemma 4 . In other words, the probability that the adversary makes a query $H_{2}\left(i, j, x_{i}, y_{j}, w_{j}, \sigma_{j}\right)$ is at most $2^{-0.97 n}+\operatorname{negl}(\kappa)$, which is negligible in $\kappa$. Since $H_{2}$ is a random oracle, we have $\operatorname{Pr}\left[F_{3,5}\right]=1 / 2+\operatorname{negl}(\kappa)$, which completes the proof.

\subsection{Type IV Adversary}

In this subsection, we prove that our AKE is secure against any PPT Type IV adversary $\mathcal{A}$.

Lemma 8. Let $n$ be a power of 2 satisfying $0.97 n \geq 2 \kappa$, prime $q>203$ satisfying $q=1 \bmod 2 n$, $\beta=\omega(\alpha \gamma n \sqrt{n \log n})$. Then, if $R L W E_{q, \alpha}$ is hard, the proposed AKE is secure against any PPT Type IV adversary $\mathcal{A}$ in the random oracle model.

Proof. We prove this lemma via a sequence of games $G_{4, l}$ for $0 \leq l \leq 4$.

Game $G_{4,0} . \mathcal{S}$ first chooses $i^{*}, j^{*} \leftarrow_{r}\{1, \ldots, N\}$ and $s_{i^{*}}, s_{j^{*}} \leftarrow_{r}\{1, \ldots, m\}$, and hopes that the adversary will choose $\operatorname{sid}^{*}=\left(\Pi, R, j^{*}, i^{*}, x_{i^{*}},\left(y_{j^{*}}, w_{j^{*}}\right)\right)$ as the test session, where $x_{i^{*}}$ is output by the $s_{i^{*} \text {-th session }}$ of party $i^{*}$, and $\left(y_{j^{*}}, w_{j^{*}}\right)$ is output by the $s_{j}^{*}$-th session of party $j^{*}$ activated by a $\operatorname{Send}_{1}\left(\Pi, R, j^{*}, i^{*}, x_{i^{*}}\right)$. Then, $\mathcal{S}$ chooses $a \leftarrow_{r} R_{q}$, honestly generates static public keys for all parties (by randomly choosing $s_{i}$ and $e_{i}$ from $\chi_{\alpha}$ ), and simulates the attack environment for $\mathcal{A}$. Specifically, $\mathcal{S}$ maintains two tables $L_{1}, L_{2}$ for the random oracles $H_{1}, H_{2}$, respectively, and answers the queries from $\mathcal{A}$ as follows:

- $H_{1}($ in $)$ : If there doesn't exist a tuple $($ in, out $)$ in the $L_{1}$ list, choose an element out $\leftarrow_{r} \chi_{\gamma}$, and add (in, out) to the $L_{1}$ list. Then, return out to $\mathcal{A}$.

- $H_{2}($ in $)$ queries: If there doesn't exist a tuple (in, out) in the $L_{2}$ list, choose an element out $\leftarrow_{r}\{0,1\}^{k}$, and add (in, out) to the $L_{2}$ list. Then, return out to $\mathcal{A}$.

- $\operatorname{Send}_{0}(\Pi, I, i, j): \mathcal{A}$ activates a new session of $i$ with intended party $j, \mathcal{S}$ proceeds as follows:

1. Sample $r_{i}, f_{i} \leftarrow_{r} \chi_{\beta}$ and compute $x_{i}=a r_{i}+2 f_{i}$;

2. Compute $c=H_{1}\left(i, j, x_{i}\right), \hat{r}_{i}=s_{i} c+r_{i}$ and $\hat{f}_{i}=e_{i} c+f_{i}$; 
3. Letting $\mathbf{z} \in \mathbb{Z}^{2 n}$ be the coefficient vector of $\hat{r}_{i}$ concatenated with the coefficient vector of $\hat{f}_{i}$, and $\mathbf{z}_{1} \in \mathbb{Z}^{2 n}$ be the coefficient vector of $s_{i} c$ concatenated with the coefficient vector of $e_{i} c$, repeat the steps $1 \sim 3$ with probability $1-\min \left(\frac{D_{\mathbb{Z}^{2 n}, \beta}(\mathbf{z})}{M D_{\mathbb{Z}^{2 n}, \beta, \mathbf{z}_{1}}(\mathbf{z})}, 1\right)$.

4. Return $x_{i}$ to $\mathcal{A}$.

- $\operatorname{Send}_{1}\left(\Pi, R, j, i, x_{i}\right): \mathcal{S}$ proceeds as follows:

$1^{\prime}$. Sample $r_{j}, f_{j} \leftarrow_{r} \chi_{\beta}$ and compute $y_{j}=a r_{j}+2 f_{j}$;

$2^{\prime}$. Compute $d=H_{1}\left(j, i, y_{j}, x_{i}\right), \hat{r}_{j}=s_{j} d+r_{j}$ and $\hat{f}_{j}=e_{j} d+f_{j}$;

$3^{\prime}$. Letting $\mathbf{z} \in \mathbb{Z}^{2 n}$ be the coefficient vector of $\hat{r}_{j}$ concatenated with the coefficient vector of $\hat{f}_{j}$, and $\mathbf{z}_{1} \in \mathbb{Z}^{2 n}$ be the coefficient vector of $s_{j} d$ concatenated with the coefficient vector of $e_{j} d$, repeat the steps $1^{\prime} \sim 3^{\prime}$ with probability $1-\min \left(\frac{D_{\mathbb{Z}^{2 n}, \beta}(\mathbf{z})}{M D_{\mathbb{Z}^{2 n}, \beta, \mathbf{z}_{1}}(\mathbf{z})}, 1\right)$;

$4^{\prime}$. Sample $g_{j} \leftarrow_{r} \chi_{\beta}$ and compute $k_{j}=\left(p_{i} c+x_{i}\right) \hat{r}_{j}+2 c g_{j}$ where $c=H_{1}\left(i, j, x_{i}\right)$;

$5^{\prime}$. Compute $w_{j}=\operatorname{Cha}\left(k_{j}\right) \in\{0,1\}^{n}$ and return $\left(y_{j}, w_{j}\right)$ to $\mathcal{A}$;

$6^{\prime}$. Compute $\sigma_{j}=\operatorname{Mod}_{2}\left(k_{j}, w_{j}\right)$ and derive the session key sk $\operatorname{sk}_{j}=H_{2}\left(i, j, x_{i}, y_{j}, w_{j}, \sigma_{j}\right)$.

- $\operatorname{Send}_{2}\left(\Pi, I, i, j, x_{i},\left(y_{j}, w_{j}\right)\right): \mathcal{S}$ computes $k_{i}$ and $\mathrm{sk}_{i}$ as follows:

5. Sample $g_{i} \leftarrow_{r} \chi_{\beta}$ and compute $k_{i}=\left(p_{j} d+y_{j}\right) \hat{r}_{i}+2 d g_{i}$ where $d=H_{1}\left(j, i, y_{j}, x_{i}\right)$;

6. Compute $\sigma_{i}=\operatorname{Mod}_{2}\left(k_{i}, w_{j}\right)$ and derive the session key sk $\operatorname{sk}_{i}=H_{2}\left(i, j, x_{i}, y_{j}, w_{j}, \sigma_{i}\right)$.

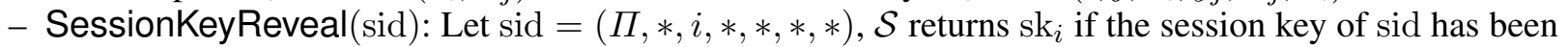
generated.

- Corrupt $(i)$ : Return the static secret key $s_{i}$ of $i$ to $\mathcal{A}$.

- Test(sid): Let sid $=\left(\Pi, I, i, j, x_{i},\left(y_{j}, w_{j}\right)\right)$, if $(i, j) \neq\left(i^{*}, j^{*}\right)$, or $x_{i}$ and $y_{j}$ are not output by the $s_{i^{*}}$-th session of $i^{*}$ and the $s_{j}^{*}$-th session of $j^{*}$, respectively, $\mathcal{S}$ aborts. Otherwise, $\mathcal{S}$ chooses $b \leftarrow_{r}\{0,1\}$ and $s k_{i}^{\prime} \leftarrow r\{0,1\}^{k}$. If $b=0, \mathcal{S}$ returns $s k_{i}^{\prime}$, else it returns the real session $\operatorname{sk}_{i}$ of sid.

Claim 23 The probability that $\mathcal{S}$ will not abort in $G_{4,0}$ is at least $\frac{1}{m^{2} N^{2}}$.

Proof. This claim directly follows from the fact that $\mathcal{S}$ randomly chooses $i^{*}, j^{*} \leftarrow{ }_{r}\{1, \ldots, N\}$ and $s_{i^{*}}, s_{j}^{*} \leftarrow_{r}\{1, \ldots, m\}$ independently from the view of $\mathcal{A}$.

Game $G_{4,1}$. $\mathcal{S}$ behaves almost the same as in $G_{4,0}$, except in the following case:

- $\operatorname{Send}_{1}\left(\Pi, R, j, i, x_{i}\right):$ If $(i, j) \neq\left(i^{*}, j^{*}\right)$, or it is not the $s_{j}^{*}$-th session of $j^{*}, \mathcal{S}$ answers the query as in Game $G_{4,0}$. Otherwise, it proceeds as follows:

$1^{\prime}$. Sample an invertible element $d \leftarrow_{r} \chi_{\gamma}$, and $\mathbf{z} \leftarrow_{r} D_{\mathbb{Z}^{2 n}, \beta}$;

$2^{\prime}$. Interpreting $\mathbf{z}$ as two ring elements $\hat{r}_{j}, \hat{f}_{j} \in R_{q}$, and define $y_{j}=a \hat{r}_{j}+2 \hat{f}_{j}-p_{j} d$.

$3^{\prime}$. Repeat the steps $1^{\prime} \sim 3^{\prime}$ with probability $1-1 / M$;

$4^{\prime}$. Abort if there is a tuple $\left(\left(j, i, y_{j}, x_{i}\right), *\right)$ in $L_{1}$. Else, add $\left(\left(j, i, y_{j}, x_{i}\right), d\right)$ into $L_{1}$. Then, sample $g_{j} \leftarrow_{r}$ $\chi_{\beta}$ and compute $k_{j}=\left(p_{i} c+x_{i}\right) \hat{r}_{j}+2 c g_{j}$ where $c=H_{1}\left(i, j, x_{i}\right)$;

$5^{\prime}$. Compute $w_{j}=\operatorname{Cha}\left(k_{j}\right) \in\{0,1\}^{n}$ and return $\left(y_{j}, w_{j}\right)$ to $\mathcal{A}$;

$6^{\prime}$. Compute $\sigma_{j}=\operatorname{Mod}_{2}\left(k_{j}, w_{j}\right)$ and derive the session key sk $\operatorname{sk}_{j}=H_{2}\left(i, j, x_{i}, y_{j}, w_{j}, \sigma_{j}\right)$.

In the following, we define $F_{4, l}$ as the event that $\mathcal{A}$ outputs a guess $b^{\prime}$ that equals to $b$ in Game $G_{4, l}$.

Claim 24 If $\beta=\omega(\alpha \gamma n \sqrt{n \log n})$ and $R L W E_{q, \beta}$ is hard, then $\operatorname{Pr}\left[F_{4,1}\right]=\operatorname{Pr}\left[F_{4,0}\right]-\operatorname{negl}(\kappa)$.

Proof. This claim can be proven via a sequence of games similar to that from $G_{1,0}$ to $G_{1,2}$, we omit the details. 
Game $G_{4,2}$. $\mathcal{S}$ behaves almost the same as in $G_{4,1}$, except for the following cases:

- $\operatorname{Send}_{0}(\Pi, I, i, j):$ If $(i, j) \neq\left(i^{*}, j^{*}\right)$, or it is not the $s_{i^{*}}$-th session of $i^{*}, \mathcal{S}$ answers the query as in Game $G_{4,1}$. Otherwise, it proceeds as follows:

1. Sample an invertible element $c \leftarrow{ }_{r} \chi_{\gamma}$, and $\mathbf{z} \leftarrow_{r} D_{\mathbb{Z}^{2 n}, \beta}$;

2. Interpreting $\mathbf{z}$ as two ring elements $\hat{r}_{i}, \hat{f}_{i} \in R_{q}$, and define $x_{i}=a \hat{r}_{i}+2 \hat{f}_{i}-p_{i} c$.

3. Repeat the steps $1 \sim 3$ with probability $1-1 / M$;

4. Abort if there is a tuple $\left(\left(i, j, x_{i}\right), *\right)$ in $L_{1}$. Else, add $\left(\left(i, j, x_{i}\right), c\right)$ into $L_{1}$. Return $x_{i}$ to $\mathcal{A}$.

- $\operatorname{Send}_{2}\left(\Pi, I, i, j, x_{i},\left(y_{j}, w_{j}\right)\right)$ : If $(i, j) \neq\left(i^{*}, j^{*}\right)$, or it is not the $s_{i^{*}}$-th session of $i^{*}, \mathcal{S}$ answers the query as in Game $G_{4,1}$. Otherwise, if $\left(y_{j}, w_{j}\right)$ is output by the $s_{j}^{*}$-th session of party $j^{*}$, let $\mathrm{sk}_{j}$ be the session key of session sid $=\left(\Pi, R, j, i, x_{i},\left(y_{j}, w_{j}\right)\right), \mathcal{S}$ sets $\operatorname{sk}_{i}=\operatorname{sk}_{j}$. Else, $\mathcal{S}$ samples $g_{i} \leftarrow_{r} \chi_{\beta}$ and computes $k_{i}=\left(p_{j} d+y_{j}\right) \hat{r}_{i}+2 d g_{i}$ where $d=H_{1}\left(j, i, y_{j}, x_{i}\right)$. Finally, it computes $\sigma_{i}=\operatorname{Mod}_{2}\left(k_{i}, w_{j}\right)$ and derive the session key $\operatorname{sk}_{i}=H_{2}\left(i, j, x_{i}, y_{j}, w_{j}, \sigma_{i}\right)$.

Claim 25 If $\beta=\omega(\alpha \gamma n \sqrt{n \log n})$ and $R L W E_{q, \beta}$ is hard, then $\operatorname{Pr}\left[F_{4,2}\right]=\operatorname{Pr}\left[F_{4,1}\right]-\operatorname{negl}(\kappa)$.

Proof. This claim can be proven via a sequence of games similar to that from $G_{1,3}$ to $G_{1,5}$, we omit the details.

Game $G_{4,3} \mathcal{S}$ behaves almost the same as in $G_{4,2}$ except in the following case:

- $\operatorname{Send}_{0}(\Pi, I, i, j)$ : If $(i, j) \neq\left(i^{*}, j^{*}\right)$, or it is not the $s_{i^{*}}$-th session of $i^{*}, \mathcal{S}$ answers as in Game $G_{4,2}$. Otherwise, it proceeds as follows:

1. Sample an invertible element $c \leftarrow_{r} \chi_{\gamma}$, and $\hat{x}_{i} \leftarrow_{r} R_{q}$;

2. Define $x_{i}=\hat{x}_{i}-p_{i} c$.

3. Repeat the steps $1 \sim 3$ with probability $1-1 / M$;

4. Abort if there is a tuple $\left(\left(i, j, x_{i}\right), *\right)$ in $L_{1}$. Else, add $\left(\left(i, j, x_{i}\right), c\right)$ into $L_{1}$. Return $x_{i}$ to $\mathcal{A}$.

- $\operatorname{Send}_{2}\left(\Pi, I, i, j, x_{i},\left(y_{j}, w_{j}\right)\right)$ : If $(i, j) \neq\left(i^{*}, j^{*}\right)$, or it is not the $s_{i^{*}}$ th session of $i^{*}$, or $\left(y_{j}, w_{j}\right)$ is output by the $s_{j}^{*}$-th session of party $j^{*}, \mathcal{S}$ behaves the same as in $G_{4,2}$. Otherwise, it proceeds as follows:

5. Randomly choose $k_{i} \leftarrow_{r} R_{q}$;

6. Compute $\sigma_{i}=\operatorname{Mod}_{2}\left(k_{i}, w_{j}\right)$ and derive the session key sk $\operatorname{sk}_{i}=H_{2}\left(i, j, x_{i}, y_{j}, w_{j}, \sigma_{i}\right)$.

Denote $Q_{4, l}$ be event that in Game $G_{4, l}$ for $l=2,3,4, \mathcal{A}$ makes a $H_{2}$ query with $\sigma_{i}$ for the $s_{i^{*}}$-th session of party $i^{*}$, when $\left(y_{j}, w_{j}^{\prime}\right)$ is output by the $s_{j}^{*}$-th session of party $j^{*}$ but $w_{j} \neq w_{j}^{\prime}$.

Claim 26 If $R L W E_{q, \beta}$ is hard, $\operatorname{Pr}\left[Q_{4,3}\right]=\operatorname{Pr}\left[Q_{4,2}\right]-\operatorname{negl}(\kappa)$, and $\operatorname{Pr}\left[F_{4,3} \mid \neg Q_{4,3}\right]=\operatorname{Pr}\left[F_{4,2} \mid \neg Q_{4,2}\right]-$ $\operatorname{negl}(\kappa)$.

Proof. The proof is similar to the proof of Claim 7, we omit the details.

Game $G_{4,4} \mathcal{S}$ behaves almost the same as in $G_{4,3}$ except in the following case:

- $\operatorname{Send}_{1}\left(\Pi, R, j, i, x_{i}\right):$ If $(i, j) \neq\left(i^{*}, j^{*}\right)$, or it is not the $s_{j}^{*}$-th session of $j^{*}, \mathcal{S}$ answers the query as in Game $G_{4,3}$. Otherwise, it proceeds as follows:

$1^{\prime}$. Sample an invertible element $d \leftarrow_{r} \chi_{\gamma}$, and $\hat{y}_{j} \leftarrow_{r} R_{q}$; 
$2^{\prime}$. Define $y_{j}=\hat{y}_{j}-p_{j} d$.

$3^{\prime}$. Repeat the steps $1^{\prime} \sim 3^{\prime}$ with probability $1-1 / M$;

$4^{\prime}$. Abort if there is a tuple $\left(\left(j, i, y_{j}, x_{i}\right), *\right)$ in $L_{1}$. Else, add $\left(\left(j, i, y_{j}, x_{i}\right), d\right)$ into $L_{1}$. Then, the simulator $\mathcal{S}$ uniformly chooses $k_{j} \leftarrow_{r} R_{q}$ at random;

$5^{\prime}$. Compute $w_{j}=\operatorname{Cha}\left(k_{j}\right) \in\{0,1\}^{n}$ and return $\left(y_{j}, w_{j}\right)$ to $\mathcal{A}$;

$6^{\prime}$. Compute $\sigma_{j}=\operatorname{Mod}_{2}\left(k_{j}, w_{j}\right)$ and derive the session $\mathrm{key} \mathrm{sk}_{j}=H_{2}\left(i, j, x_{i}, y_{j}, w_{j}, \sigma_{j}\right)$.

Claim 27 Let $n$ be a power of 2 satisfying $0.97 n \geq 2 \kappa$, prime $q>203$ satisfying $q=1 \bmod 2 n$, $\beta=$ $\omega(\alpha \gamma n \sqrt{n \log n})$. Then, if $R L W E_{q, \alpha}$ is hard, Game $G_{4,3}$ and $G_{4,4}$ are computationally indistinguishable. In particular, we have $\operatorname{Pr}\left[Q_{4,4}\right]=\operatorname{Pr}\left[Q_{4,3}\right]$, and $\operatorname{Pr}\left[F_{4,4} \mid \neg Q_{4,4}\right]=\operatorname{Pr}\left[F_{4,3} \mid \neg Q_{4,3}\right]-\operatorname{negl}(\kappa)$.

Proof. The proof is similar to the proof of Claim 8, we omit the details.

Claim 28 If $0.97 n>2 \kappa$, we have $\operatorname{Pr}\left[Q_{4,4}\right]=\operatorname{negl}(\kappa)$.

Proof. The proof is similar to the proof of Claim 9, we omit the details.

Claim $29 \operatorname{Pr}\left[F_{4,4} \mid \neg Q_{4,4}\right]=1 / 2+\operatorname{negl}(\kappa)$.

Proof. The proof is similar to the proof of Claim 10, we omit the details.

Combining the claims 23 29, we have that Lemma 8 follows.

\subsection{Type V Adversary}

In this subsection, we prove that our AKE is secure against any PPT Type $\mathbf{V}$ adversary $\mathcal{A}$.

Lemma 9. Let $n$ be a power of 2 satisfying $0.97 n \geq 2 \kappa$, prime $q>203$ satisfying $q=1 \bmod 2 n$, $\beta=\omega(\alpha \gamma n \sqrt{n \log n})$. Then, if $R L W E_{q, \alpha}$ is hard, the proposed AKE is secure against any PPT Type $\boldsymbol{V}$ adversary $\mathcal{A}$ in the random oracle model.

Proof. We prove this lemma via a sequence of games $G_{5, l}$ for $0 \leq l \leq 4$.

Game $G_{5,0}$. $\mathcal{S}$ chooses $i^{*}, j^{*} \leftarrow r\{1, \ldots, N\}$ and $s_{i^{*}}, s_{j^{*}} \leftarrow_{r}\{1, \ldots, m\}$, and hopes that the adversary will choose $\operatorname{sid}^{*}=\left(\Pi, R, j^{*}, i^{*}, x_{i^{*}},\left(y_{j^{*}}, w_{j^{*}}\right)\right)$ as the test session, where $x_{i^{*}}$ is output by the $s_{i^{*}}$-th session of party $i^{*}$, and $\left(y_{j} *, w_{j^{*}}\right)$ is output by the $s_{j}^{*}$-th session of party $j^{*}$ activated by a $\operatorname{Send}_{1}\left(\Pi, R, j^{*}, i^{*}, x_{i^{*}}\right)$. Then, $\mathcal{S}$ chooses $a \leftarrow_{r} R_{q}$, honestly generates static public keys for all parties (by randomly choosing $s_{i}$ and $e_{i}$ from $\chi_{\alpha}$ ), and simulates the attack environment for $\mathcal{A}$. Specifically, $\mathcal{S}$ maintains two tables $L_{1}, L_{2}$ for the random oracles $H_{1}, H_{2}$, respectively, and answers the queries from $\mathcal{A}$ as follows:

- $H_{1}(i n)$ : If there doesn't exist a tuple $($ in,$o u t)$ in the $L_{1}$ list, choose an element out $\leftarrow_{r} \chi_{\gamma}$, and add (in, out) to the $L_{1}$ list. Then, return out to $\mathcal{A}$.

- $H_{2}($ in $)$ queries: If there doesn't exist a tuple $($ in, out $)$ in the $L_{2}$ list, choose an element out $\leftarrow_{r}\{0,1\}^{k}$, and add (in, out) to the $L_{2}$ list. Then, return out to $\mathcal{A}$.

- $\operatorname{Send}_{0}(\Pi, I, i, j): \mathcal{A}$ activates a new session of $i$ with intended party $j, \mathcal{S}$ proceeds as follows:

1. Sample $r_{i}, f_{i} \leftarrow_{r} \chi_{\beta}$ and compute $x_{i}=a r_{i}+2 f_{i}$;

2. Compute $c=H_{1}\left(i, j, x_{i}\right), \hat{r}_{i}=s_{i} c+r_{i}$ and $\hat{f}_{i}=e_{i} c+f_{i}$; 
3. Letting $\mathbf{z} \in \mathbb{Z}^{2 n}$ be the coefficient vector of $\hat{r}_{i}$ concatenated with the coefficient vector of $\hat{f}_{i}$, and $\mathbf{z}_{1} \in \mathbb{Z}^{2 n}$ be the coefficient vector of $s_{i} c$ concatenated with the coefficient vector of $e_{i} c$, repeat the steps $1 \sim 3$ with probability $1-\min \left(\frac{D_{\mathbb{Z}^{2 n}, \beta}(\mathbf{z})}{M D_{\mathbb{Z}^{2 n}, \beta, \mathbf{z}_{1}}(\mathbf{z})}, 1\right)$;

4. Return $x_{i}$ to $\mathcal{A}$.

- $\operatorname{Send}_{1}\left(\Pi, R, j, i, x_{i}\right): \mathcal{S}$ proceeds as follows:

$1^{\prime}$. Sample $r_{j}, f_{j} \leftarrow_{r} \chi_{\beta}$ and compute $y_{j}=a r_{j}+2 f_{j}$;

$2^{\prime}$. Compute $d=H_{1}\left(j, i, y_{j}, x_{i}\right), \hat{r}_{j}=s_{j} d+r_{j}$ and $\hat{f}_{j}=e_{j} d+f_{j}$;

$3^{\prime}$. Letting $\mathbf{z} \in \mathbb{Z}^{2 n}$ be the coefficient vector of $\hat{r}_{j}$ concatenated with the coefficient vector of $\hat{f}_{j}$, and $\mathbf{z}_{1} \in \mathbb{Z}^{2 n}$ be the coefficient vector of $s_{j} d$ concatenated with the coefficient vector of $e_{j} d$, repeat the steps $1^{\prime} \sim 3^{\prime}$ with probability $1-\min \left(\frac{D_{\mathbb{Z}^{2 n}, \beta}(\mathbf{z})}{M D_{\mathbb{Z}^{2 n}, \beta, \mathbf{z}_{1}}(\mathbf{z})}, 1\right)$;

$4^{\prime}$. Sample $g_{j} \leftarrow_{r} \chi_{\beta}$ and compute $k_{j}=\left(p_{i} c+x_{i}\right) \hat{r}_{j}+2 c g_{j}$ where $c=H_{1}\left(i, j, x_{i}\right)$;

$5^{\prime}$. Compute $w_{j}=\operatorname{Cha}\left(k_{j}\right) \in\{0,1\}^{n}$ and return $\left(y_{j}, w_{j}\right)$ to $\mathcal{A}$;

$6^{\prime}$. Compute $\sigma_{j}=\operatorname{Mod}_{2}\left(k_{j}, w_{j}\right)$ and derive the session key sk $\mathrm{sk}_{j}=H_{2}\left(i, j, x_{i}, y_{j}, w_{j}, \sigma_{j}\right)$.

- $\operatorname{Send}_{2}\left(\Pi, I, i, j, x_{i},\left(y_{j}, w_{j}\right)\right): \mathcal{S}$ computes $k_{i}$ and $\mathrm{sk}_{i}$ as follows:

5. Sample $g_{i} \leftarrow_{r} \chi_{\beta}$ and compute $k_{i}=\left(p_{j} d+y_{j}\right) \hat{r}_{i}+2 d g_{i}$ where $d=H_{1}\left(j, i, y_{j}, x_{i}\right)$;

6. Compute $\sigma_{i}=\operatorname{Mod}_{2}\left(k_{i}, w_{j}\right)$ and derive the session key sk $\operatorname{sk}_{i}=H_{2}\left(i, j, x_{i}, y_{j}, w_{j}, \sigma_{i}\right)$.

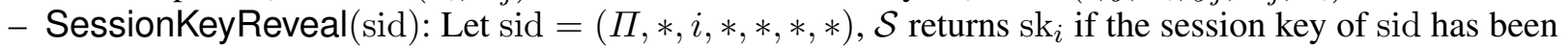
generated.

- Corrupt $(i)$ : Return the static secret key $s_{i}$ of $i$ to $\mathcal{A}$.

- Test(sid): Let sid $=\left(\Pi, I, i, j, x_{i},\left(y_{j}, w_{j}\right)\right)$, if $(i, j) \neq\left(i^{*}, j^{*}\right)$, or $x_{i}$ and $y_{j}$ are not output by the $s_{i^{*}}$-th session of $i^{*}$ and the $s_{j}^{*}$-th session of $j^{*}$, respectively, $\mathcal{S}$ aborts. Otherwise, $\mathcal{S}$ chooses $b \leftarrow_{r}\{0,1\}$ and $s k_{i}^{\prime} \leftarrow r\{0,1\}^{k}$. If $b=0, \mathcal{S}$ returns $s k_{i}^{\prime}$, else it returns the real session $\operatorname{sk}_{i}$ of sid.

Claim 30 The probability that $\mathcal{S}$ will not abort in $G_{5,0}$ with probability at least $\frac{1}{m^{2} N^{2}}$.

Proof. This claim directly follows from the fact that $\mathcal{S}$ randomly chooses $i^{*}, j^{*} \leftarrow{ }_{r}\{1, \ldots, N\}$ and $s_{i^{*}}, s_{j}^{*} \leftarrow r\{1, \ldots, m\}$ independently from the view of $\mathcal{A}$.

Game $G_{5,1}$. $\mathcal{S}$ behaves almost the same as in $G_{5,0}$, except in the following case:

- $\operatorname{Send}_{1}\left(\Pi, R, j, i, x_{i}\right):$ If $(i, j) \neq\left(i^{*}, j^{*}\right)$, or it is not the $s_{j}^{*}$-th session of $j^{*}, \mathcal{S}$ answers the query as in Game $G_{5,0}$. Otherwise, it proceeds as follows:

$1^{\prime}$. Sample an invertible element $d \leftarrow_{r} \chi_{\gamma}$, and $\mathbf{z} \leftarrow_{r} D_{\mathbb{Z}^{2 n}, \beta}$;

$2^{\prime}$. Interpreting $\mathbf{z}$ as two ring elements $\hat{r}_{j}, \hat{f}_{j} \in R_{q}$, and define $y_{j}=a \hat{r}_{j}+2 \hat{f}_{j}-p_{j} d$.

$3^{\prime}$. Repeat the steps $1^{\prime} \sim 3^{\prime}$ with probability $1-1 / M$;

$4^{\prime}$. Abort if there is a tuple $\left(\left(j, i, y_{j}, x_{i}\right), *\right)$ in $L_{1}$. Else, add $\left(\left(j, i, y_{j}, x_{i}\right), d\right)$ into $L_{1}$. Then, sample $g_{j} \leftarrow_{r}$ $\chi_{\beta}$ and compute $k_{j}=\left(p_{i} c+x_{i}\right) \hat{r}_{j}+2 c g_{j}$ where $c=H_{1}\left(i, j, x_{i}\right)$;

$5^{\prime}$. Compute $w_{j}=\operatorname{Cha}\left(k_{j}\right) \in\{0,1\}^{n}$ and return $\left(y_{j}, w_{j}\right)$ to $\mathcal{A}$;

$6^{\prime}$. Compute $\sigma_{j}=\operatorname{Mod}_{2}\left(k_{j}, w_{j}\right)$ and derive the session key sk $\operatorname{sk}_{j}=H_{2}\left(i, j, x_{i}, y_{j}, w_{j}, \sigma_{j}\right)$.

In the following, let $F_{5, l}$ denote the event that $\mathcal{A}$ outputs a guess $b^{\prime}$ that equals to $b$ in Game $G_{5, l}$.

Claim 31 If $\beta=\omega(\alpha \gamma n \sqrt{n \log n})$ and $R L W E_{q, \beta}$ is hard, then $\operatorname{Pr}\left[F_{5, l}\right]=\operatorname{Pr}\left[F_{5,0}\right]-\operatorname{negl}(\kappa)$.

Proof. This claim can be proven via a sequence of games similar to that from $G_{1,0}$ to $G_{1,2}$, we omit the details. 
Game $G_{5,2}$. $\mathcal{S}$ behaves almost the same as in $G_{5,1}$, except for the following cases:

- $\operatorname{Send}_{0}(\Pi, I, i, j)$ : If $(i, j) \neq\left(i^{*}, j^{*}\right)$, or it is not the $s_{i^{*}}$-th session of $i^{*}, \mathcal{S}$ answers the query as in Game $G_{5,1}$. Otherwise, it proceeds as follows:

1. Sample an invertible element $c \leftarrow r \chi_{\gamma}$, and $\mathbf{z} \leftarrow r D_{\mathbb{Z}^{2 n}, \beta}$;

2. Interpreting $\mathbf{z}$ as two ring elements $\hat{r}_{i}, \hat{f}_{i} \in R_{q}$, and define $x_{i}=a \hat{r}_{i}+2 \hat{f}_{i}-p_{i} c$.

3. Repeat the steps $1 \sim 3$ with probability $1-1 / M$;

4. Abort if there is a tuple $\left(\left(i, j, x_{i}\right), *\right)$ in $L_{1}$. Else, add $\left(\left(i, j, x_{i}\right), c\right)$ into $L_{1}$. Return $x_{i}$ to $\mathcal{A}$.

Claim 32 If $\beta=\omega(\alpha \gamma n \sqrt{n \log n})$ and $R L W E_{q, \beta}$ is hard, then $\operatorname{Pr}\left[F_{5,2}\right]=\operatorname{Pr}\left[F_{5,1}\right]-\operatorname{negl}(\kappa)$.

Proof. This claim can be proven via a sequence of games similar to that from $G_{1,2}$ to $G_{1,4}$, we omit the details.

Game $G_{5,3} \mathcal{S}$ behaves almost the same as in $G_{5,2}$ except in the following case:

- $\operatorname{Send}_{0}(\Pi, I, i, j)$ : If $(i, j) \neq\left(i^{*}, j^{*}\right)$, or it is not the $s_{i^{*}}$-th session of $i^{*}, \mathcal{S}$ answers as in Game $G_{5,2}$. Otherwise, it proceeds as follows:

1. Sample an invertible element $c \leftarrow_{r} \chi_{\gamma}$, and $\hat{x}_{i} \leftarrow_{r} R_{q}$;

2. Define $x_{i}=\hat{x}_{i}-p_{i} c$.

3. Repeat the steps $1 \sim 3$ with probability $1-1 / M$;

4. Abort if there is a tuple $\left(\left(i, j, x_{i}\right), *\right)$ in $L_{1}$. Else, add $\left(\left(i, j, x_{i}\right), c\right)$ into $L_{1}$. Return $x_{i}$ to $\mathcal{A}$.

- Send ${ }_{2}\left(\Pi, I, i, j, x_{i},\left(y_{j}, w_{j}\right)\right)$ : If $(i, j) \neq\left(i^{*}, j^{*}\right)$, or it is not the $s_{i^{*}}$-th session of $i^{*}$, or $y_{j}$ is output by the $s_{j}^{*}$-th session of party $j^{*}, \mathcal{S}$ behaves the same as in $G_{5,2}$. Otherwise, it proceeds as follows:

5. Randomly choose $k_{i} \leftarrow{ }_{r} R_{q}$;

6. Compute $\sigma_{i}=\operatorname{Mod}_{2}\left(k_{i}, w_{j}\right)$ and derive the session key sk $\operatorname{sk}_{i}=H_{2}\left(i, j, x_{i}, y_{j}, w_{j}, \sigma_{i}\right)$.

Claim $33 \operatorname{Pr}\left[F_{5,3}\right]=\operatorname{Pr}\left[F_{5,2}\right]-\operatorname{negl}(\kappa)$.

Proof. To prove this claim, we have to distinguish two cases: 1) $y_{j}$ is output by party $j=j^{*}$ at session $s_{j} \neq s_{j}^{*}$ in response to a $\operatorname{Send}_{1}\left(\Pi, R, j, i^{*}, x_{i}\right)$ query; 2$) y_{j}$ is not output by party $j=j^{*}$ at any session in response to a $\operatorname{Send}_{1}\left(\Pi, R, j, i^{*}, x_{i}\right)$ query. For the first case, the adversary just replays the messages to activate two sessions at party $j$. This situation is very similar to a Type I adversary, and one can prove this calim via a sequence of games as that from Game $G_{1,5}$ to $G_{1,7}$. While for the second case, this claim can also be proven via a sequence of games as that from Game $G_{2,3}$ to $G_{2,5}$. We omit the details here.

Game $G_{5,4}$. $\mathcal{S}$ behaves almost the same as in $G_{5,3}$, except in the following case:

- $\operatorname{Send}_{1}\left(\Pi, R, j, i, x_{i}\right)$ : If $(i, j) \neq\left(i^{*}, j^{*}\right)$, or it is not the $s_{j}^{*}$-th session of $j^{*}, \mathcal{S}$ answers the query as in Game $G_{5,3}$. Otherwise, it proceeds as follows:

$1^{\prime}$. Sample an invertible element $d \leftarrow_{r} \chi_{\gamma}$, and $\hat{y}_{j} \leftarrow_{r} R_{q}$;

$2^{\prime}$. Define $y_{j}=\hat{y}_{j}-p_{j} d$.

$3^{\prime}$. Repeat the steps $1^{\prime} \sim 3^{\prime}$ with probability $1-1 / M$;

$4^{\prime}$. Abort if there is a tuple $\left(\left(j, i, y_{j}, x_{i}\right), *\right)$ in $L_{1}$. Else, add $\left(\left(j, i, y_{j}, x_{i}\right), d\right)$ into $L_{1}$. Then, the simulator $\mathcal{S}$ uniformly chooses $k_{j} \leftarrow_{r} R_{q}$ at random; 
$5^{\prime}$. Compute $w_{j}=\mathrm{Cha}\left(k_{j}\right) \in\{0,1\}^{n}$ and return $\left(y_{j}, w_{j}\right)$ to $\mathcal{A}$;

$6^{\prime}$. Compute $\sigma_{j}=\operatorname{Mod}_{2}\left(k_{j}, w_{j}\right)$ and derive the session $\operatorname{key~sk}_{j}=H_{2}\left(i, j, x_{i}, y_{j}, w_{j}, \sigma_{j}\right)$.

Claim 34 Let $n$ be a power of 2 satisfying $0.97 n \geq 2 \kappa$, prime $q>203$ satisfying $q=1 \bmod 2 n$, $\beta=$ $\omega(\alpha \gamma n \sqrt{n \log n})$. Then, if $R L W E_{q, \alpha}$ is hard, Game $G_{5,3}$ and $G_{5,4}$ are computationally indistinguishable. In particular, we have that $\operatorname{Pr}\left[F_{5,4}\right]=\operatorname{Pr}\left[F_{5,3}\right]-\operatorname{negl}(\kappa)$.

Proof. The proof is similar to Claim 8, we omit the details.

Claim 35 If $0.97 n>2 \kappa, \operatorname{Pr}\left[F_{5,4}\right]=1 / 2+\operatorname{negl}(\kappa)$.

Proof. Let $\left(y_{j}, w_{j}\right)$ be output by the $s_{j}^{*}$-th session of party $j=j^{*}$, we have $\operatorname{sk}_{j}=H_{2}\left(i, j, x_{i}, y_{j}, w_{j}, \sigma_{j}\right)$, where $\sigma_{j}=\operatorname{Mod}_{2}\left(k_{j}, w_{j}\right)$. Note that in $G_{5,4}, k_{j}$ is randomly chosen from the uniform distribution over $R_{q}$, we have $\sigma_{j} \in\{0,1\}^{n}$ (conditioned on $w_{j}$ ) has min-entropy at least $0.97 n$ by Lemma 4 . Thus, the probability that $\mathcal{A}$ has made a $H_{2}$ query with $\sigma_{j}$ is less than $2^{-0.97 n}+\operatorname{negl}(\kappa)$. This claim follows from the fact that if the adversary doesn't make a query with $\sigma_{j}$ exactly, the distribution of $\operatorname{sk}_{j}$ is uniform over $\{0,1\}^{k}$ due to the random oracle property of $H_{2}$, i.e., $\operatorname{Pr}\left[F_{5,4}\right]=1 / 2+\operatorname{negl}(\kappa)$.

Combining the claims 30 35, we have that Lemma 9 follows.

\section{One-Pass Protocol from Ring-LWE}

As MQV [57] and HMQV [46], our AKE protocol has a one-pass variant, which only consists a single message from one party to the other. Let $a \in R_{q}$ be the global public parameter uniformly chosen from $R_{q}$ at random, and $M$ be a constant. Let $p_{i}=a s_{i}+2 e_{i} \in R_{q}$ be party $i$ 's static public key, where $\left(s_{i}, e_{i}\right)$ is the corresponding static secret key; both $s_{i}$ and $e_{i}$ are taken from the distribution $\chi_{\alpha}$. Similarly, party $j$ has static public key $p_{j}=a s_{j}+2 e_{j}$ and static secret key $\left(s_{j}, e_{j}\right)$. The other parameters and notations used in this section are the same as before.

Initiation Party $i$ proceeds as follows:

1. Sample $r_{i}, f_{i} \leftarrow_{r} \chi_{\beta}$ and compute $x_{i}=a r_{i}+2 f_{i}$;

2. Compute $c=H_{1}\left(i, j, x_{i}\right), \hat{r}_{i}=s_{i} c+r_{i}$ and $\hat{f}_{i}=e_{i} c+f_{i}$;

3. Letting $\mathbf{z} \in \mathbb{Z}^{2 n}$ be the coefficient vector of $\hat{r}_{i}$ concatenated with the coefficient vector of $\hat{f}_{i}$, and $\mathbf{z}_{1} \in \mathbb{Z}^{2 n}$ be the coefficient vector of $s_{i} c$ concatenated with the coefficient vector of $e_{i} c$, repeat the steps $1 \sim 3$ with probability $1-\min \left(\frac{D_{\mathbb{Z}^{2 n}, \beta}(\mathbf{z})}{M D_{\mathbb{Z}^{2 n}, \beta, \mathbf{z}_{1}}(\mathbf{z})}, 1\right)$.

4. Sample $g_{i} \leftarrow_{r} \chi_{\beta}$ and compute $k_{i}=p_{j} \hat{r}_{i}+2 g_{i}$ where $c=H_{1}\left(i, j, x_{i}\right)$;

5. Compute $w_{i}=\mathrm{Cha}\left(k_{i}\right) \in\{0,1\}^{n}$ and send $\left(y_{i}, w_{i}\right)$ to party $j$;

6. Compute $\sigma_{i}=\operatorname{Mod}_{2}\left(k_{i}, w_{i}\right)$ and derive the session key sk ${ }_{i}=H_{2}\left(i, j, x_{i}, w_{i}, \sigma_{i}\right)$.

Finish Party $j$ receives the pair $\left(x_{i}, w_{i}\right)$ from party $i$, and proceeds as follows:

$1^{\prime}$. Sample $g_{j} \leftarrow_{r} \chi_{\alpha}$ and compute $k_{j}=\left(p_{i} c+x_{i}\right) s_{j}+2 c g_{j}$ where $c=H_{1}\left(i, j, x_{i}\right)$;

$2^{\prime}$. Compute $\sigma_{j}=\operatorname{Mod}_{2}\left(k_{j}, w_{i}\right)$ and derive the session key $\operatorname{sk}_{j}=H_{2}\left(i, j, x_{i}, w_{i}, \sigma_{j}\right)$.

The correctness of the protocol simply follows from the fact that $k_{i}=p_{j} \hat{r}_{i}+2 g_{i}=\left(a s_{j}+2 e_{j}\right)\left(s_{i} c+\right.$ $\left.r_{i}\right)+2 g_{i} \approx a\left(s_{i} c+r_{i}\right) s_{j}+2\left(e_{i} c+f_{i}\right) s_{j}+2 c g_{j}=k_{j}$. The security of the protocol cannot be proven in the BR model with party corruption, since the one-pass protocol inherently can never provide wPFS due to the lack of message from the receiver $j$. Besides, the protocol cannot prevent the adversary from replaying a previous message, even though this can be detected in practice by using other measures such as keeping 
a state or using synchronized time. However, we can prove it in a weak model similar to [46] which avoids the (above) inherent insufficiencies for one-pass protocol. Since the proof goes the lines with the previous one, we omit the details.

Finally, we remark that the one-pass protocol can essentially be used as a KEM, and can be transformed into a CCA encryption in the random oracle model by combining it with a CPA-secure symmetric-key encryption together with a MAC algorithm in a standard way (where both keys are derived from the session key in the one-pass protocol). The resulting encryption has two interesting properties: 1) it allows the receiver to verify the sender's identity, but no one else can verify it (since only the receiver can compute the session key, i.e., it provides some kind of sender authentication); 2) the sender can deny having created such a ciphertext, because the receiver can also create such a ciphertext by itself (i.e., it is a deniable encryption).

\section{Concrete Parameters and Timings}

In this section, we present concrete choices of parameters, and the timings in a proof-of-concept implementation. Our selection of parameters for our AKE protocols can be found in Table 2. Those parameters were chosen such that the correctness property is satisfied with high probability and with the choice of different levels of security.

For correctness we must satisfy that the error term $\left\|\widetilde{g}_{i}-\widetilde{g}_{j}\right\|_{\infty}<q / 8$. Note that $\widetilde{g}_{i}=\left(e_{j} d+f_{j}\right)\left(s_{i} c+\right.$ $\left.r_{i}\right)+d g_{i}$, and $\widetilde{g}_{j}=\left(e_{i} c+f_{i}\right)\left(s_{j} d+r_{j}\right)+c g_{j}$, where $e_{i}, e_{j} \leftarrow_{r} \chi_{\alpha}, c, d \leftarrow_{r} \chi_{\gamma}$, and $f_{i}, f_{j}, r_{i}, r_{j}, g_{i}, g_{j} \leftarrow_{r} \chi_{\beta}$. Due to the symmetry, we only estimate the size of $\left\|\widetilde{g}_{i}\right\|_{\infty}$. At this point, we use the following fact about the product of two Gaussian distributed random values (as stated in [9]). Let $x \in R$ and $y \in R$ be two polynomials whose coefficients are distributed according to a discrete Gaussian distribution with standard deviation $\sigma$ and $\tau$, respectively. The individual coefficients of the product $x y$ are then (approximately) normally distributed around zero with standard deviation $\sigma \tau \sqrt{n}$ where $n$ is the degree of the polynomial.

In our case, it means that we have $\left\|\left(e_{j} d+f_{j}\right)\left(s_{i} c+r_{i}\right)\right\|_{\infty} \leq 6 \beta^{2} \sqrt{n}$ and $\left\|d g_{i}\right\|_{\infty} \leq 6 \gamma \beta \sqrt{n}$ with overwhelming probability ( $\operatorname{since} \operatorname{erfc}(6)$ is about $2^{-55}$ ). Note that the distributions of $e_{j} d+f_{j}$ and $s_{i} c+r_{i}$ are both according to $\chi_{\beta}$ since we use the rejection sampling in the protocol. Now, to choose an appropriate $\beta$ we set $d=1 / 2$ in Lemma 1 such that $\left\|e_{j} d\right\|,\left\|s_{i} c\right\| \leq 1 / 2 \alpha \gamma n$ with probability at most $2 \cdot 0.943^{-n}$. Hence, for $n \geq 1024$, we get a potential decryption error with only a probability about $2^{-87}$. In order to make the rejection sampling work, it is sufficient to set $\beta \geq \tau * 1 / 2 \alpha \gamma n=1 / 2 \tau \alpha \gamma n$ for some constant $\tau$ (which is much better than the worst-case bound $\beta=\omega(\alpha \gamma \sqrt{n \log n})$ in Theorem 1). For instance, if $\tau=12$, we have an expect number of rejection sampling about $M=2.72$ and a statistical distance about $\frac{2^{-100}}{M}$ by Theorem 1 . For such a choice of $\beta$, we can safely assume that $\left\|\widetilde{g}_{i}\right\|_{\infty} \leq 6 \beta^{2} \sqrt{n}+6 \gamma \beta \sqrt{n} \leq 7 \beta^{2} \sqrt{n}$. Thus, it is enough to set $16 * 7 \beta^{2} \sqrt{n}<q$ for correctness of the protocol.

Though the Ring-LWE problem enjoys a worst-case connection to some hard problems (e.g., SIVP [54]) on ideal lattices, the connection as summarized in Proposition 1 seems less powerful to estimate the actual security for concrete choices of parameters. In order to assess the concrete security of our parameters, we use the approach of [21], which investigates the two most efficient ways to solve the underlying (R)LWE problem, namely the embedding and decoding attacks. As opposed to [21], the decoding attack is more efficient against our instances because in RLWE with $m \geq 2 n$ one typically is close to the optimal attack dimension for the corresponding attacks. The decoding attack first uses a lattice reduction algorithm, such as BKZ [63] / BKZ 2.0 [18] and then applies a decoding algorithm, such as Babai's nearest plane [2], Lindner and Peikert's nearest planes [50], or Liu and Nguyen's pruned enumeration approach [51]. Finally, the closest vector is returned which coincides with the error polynomial, and the secret polynomial is recovered. 
Table 2. Choices of Parameters (The bound $6 \alpha$ with $\operatorname{erfc}(6) \approx 2^{-55}$ is used to estimate the size of secret keys)

\begin{tabular}{|c|c|c|c|c|c|c|c|c|c|c|c|}
\hline \multirow{2}{*}{ Protocol } & \multirow{2}{*}{$\begin{array}{l}\text { Choice of } \\
\text { Parameters }\end{array}$} & \multirow{2}{*}{$n$} & \multirow{2}{*}{ Security } & \multirow{2}{*}{$\alpha$} & \multirow{2}{*}{$\tau$} & \multirow{2}{*}{$\log \beta$} & \multirow{2}{*}{$\log q$ (bits) } & \multicolumn{4}{|c|}{ Size $(\mathrm{KB})$} \\
\hline & & & & & & & & $\mathrm{pk}$ & sk (expt.) & init. msg & resp. msg \\
\hline \multirow{4}{*}{ Two-pass } & $\mathrm{I}_{1}$ & \multirow{2}{*}{1024} & 80 bits & 3.397 & 12 & 16.1 & 45 & $5.625 \mathrm{~KB}$ & $1.5 \mathrm{~KB}$ & $5.625 \mathrm{~KB}$ & $5.75 \mathrm{~KB}$ \\
\hline & $\mathrm{I}_{2}$ & & 75 bits & 3.397 & 24 & 17.1 & 47 & $5.875 \mathrm{~KB}$ & $1.5 \mathrm{~KB}$ & $5.875 \mathrm{~KB}$ & $6.0 \mathrm{~KB}$ \\
\hline & $\mathrm{II}_{1}$ & \multirow{2}{*}{2048} & 230 bits & 3.397 & 12 & 17.1 & 47 & $11.75 \mathrm{~KB}$ & $3.0 \mathrm{~KB}$ & $11.75 \mathrm{~KB}$ & $12.0 \mathrm{~KB}$ \\
\hline & $\mathrm{II}_{2}$ & & 210 bits & 3.397 & 36 & 18.7 & 50 & $12.50 \mathrm{~KB}$ & $3.0 \mathrm{~KB}$ & $12.50 \mathrm{~KB}$ & $12.75 \mathrm{~KB}$ \\
\hline \multirow{4}{*}{ One-pass } & $\mathrm{III}_{1}$ & \multirow{2}{*}{1024} & 160 bits & 3.397 & 12 & 16.1 & 30 & $3.75 \mathrm{~KB}$ & $1.5 \mathrm{~KB}$ & $3.75 \mathrm{~KB}$ & $3.875 \mathrm{~KB}$ \\
\hline & $\mathrm{III}_{2}$ & & 140 bits & 3.397 & 36 & 17.7 & 32 & $4.0 \mathrm{~KB}$ & $1.5 \mathrm{~KB}$ & $4.0 \mathrm{~KB}$ & $4.125 \mathrm{~KB}$ \\
\hline & $\mathrm{IV}_{1}$ & & 360 bits & 3.397 & 12 & 17.1 & 32 & $8.0 \mathrm{~KB}$ & $3.0 \mathrm{~KB}$ & $8.0 \mathrm{~KB}$ & $8.25 \mathrm{~KB}$ \\
\hline & $\mathrm{IV}_{2}$ & & 350 bits & 3.397 & 36 & 18.7 & 33 & $8.25 \mathrm{~KB}$ & $3.0 \mathrm{~KB}$ & $8.25 \mathrm{~KB}$ & $8.5 \mathrm{~KB}$ \\
\hline
\end{tabular}

As recommended in [50,35], it is enough to set the Gaussian parameter $\alpha \geq 3.2$ so that the discrete Gaussian $D_{\mathbb{Z}^{n}, \alpha}$ approximates the continuous Gaussian $D_{\alpha}$ extremely well ${ }^{9}$. In our experiment, we fix $\alpha=3.397$ for a better performance of the Gaussian sampling algorithm in [30]. As for the choices of $\gamma$, we set $\gamma=\alpha$ for simplicity (actually such a choice in our experiments works very well: no rejection happened for 1000 times hash evaluations). In Table 1, we set all other parameters $\beta, n, q$ for our two-pass protocol to satisfy the correctness condition. We also give the parameter choices of our one-pass protocol (in this case, we can save a factor of $\beta$ in $q$ due to the asymmetry). Note that $n$ is required to be a power of 2 in our protocol (i.e., it is very sparsely distributed ${ }^{10}$ ), we present several candidate choices of parameters for $n=1024,2048$, and estimate the sizes of public keys, secret keys, and communication overheads in Table 2.

Table 3. Timings of proof-of-concept implementations in ms.

\begin{tabular}{|c|c|c|c|c|c|}
\hline Protocol & Parameters & $\tau$ & Initiation & Response & Finish \\
\hline \multirow{4}{*}{ Two-pass } & $\mathrm{I}_{1}$ & 12 & $22.05 \mathrm{~ms}$ & $30.61 \mathrm{~ms}$ & $4.35 \mathrm{~ms}$ \\
\cline { 2 - 6 } & $\mathrm{I}_{2}$ & 24 & $14.26 \mathrm{~ms}$ & $19.18 \mathrm{~ms}$ & $4.41 \mathrm{~ms}$ \\
\cline { 2 - 6 } & $\mathrm{II}_{1}$ & 12 & $49.77 \mathrm{~ms}$ & $60.31 \mathrm{~ms}$ & $9.44 \mathrm{~ms}$ \\
\cline { 2 - 6 } & $\mathrm{II}_{2}$ & 36 & $25.40 \mathrm{~ms}$ & $36.96 \mathrm{~ms}$ & $9.59 \mathrm{~ms}$ \\
\hline
\end{tabular}

\begin{tabular}{|c|c|c|c|c|}
\hline Protocol & Parameters & $\tau$ & Initiation & Finish \\
\hline \multirow{4}{*}{ One-pass } & $\mathrm{III}_{1}$ & 12 & $26.17 \mathrm{~ms}$ & $3.64 \mathrm{~ms}$ \\
\cline { 2 - 5 } & $\mathrm{III}_{2}$ & 36 & $14.57 \mathrm{~ms}$ & $3.70 \mathrm{~ms}$ \\
\cline { 2 - 5 } & $\mathrm{IV}_{1}$ & 12 & $53.78 \mathrm{~ms}$ & $7.75 \mathrm{~ms}$ \\
\cline { 2 - 5 } & $\mathrm{IV}_{2}$ & 36 & $32.28 \mathrm{~ms}$ & $7.94 \mathrm{~ms}$ \\
\hline
\end{tabular}

We implement our AKE protocol by using the NTL library compiled with the option NTL_GMP_LIP=on (i.e., building NTL using the GNU Multi-Precision package). The implementations are written in C++ without any parallel computations or multi-threads programming techniques. The program is run on a Dell Optiplex 780 computer with Ubuntu 12.04 TLS 64-bit system, equipped with a 2.83GHz Intel Core 2 Quad CPU and 3.8GB RAM. We use a $n$-dimensional Fast Fourier Transform (FFT) for the multiplications of two ring elements [20,53]. We use the CDT algorithm [60] as a tool for hashing to $D_{\mathbb{Z}^{n}, \gamma}$ and sampling from $D_{\mathbb{Z}^{n}, \alpha}$, but use the DDLL algorithm [30] for sampling from $D_{\mathbb{Z}^{n}, \beta}$ (because the CDT algorithm has to store large precomputed values for a big $\beta$ ). In Table 3, we present the timings of each operation, and the figures represent the averaged timing (in millisecond, ms) for 1000 executions. Since our protocols also allow some kind of precomputations such as sampling Gaussian distributions offline, the timings can be greatly reduced if one consider it in practice. Finally, we note that our implementation has not undergone any real optimization, and it can much improved in practice.

\footnotetext{
9 Only $\alpha$ is considered because $\beta \gg \alpha$, and the (R-)LWE problem becomes harder as $\alpha$ grows bigger (for a fixed modulus $q$ ).

${ }^{10}$ We remark such a choice of $n$ is not necessary, but it gives a simple analysis and implementation. In practice, one might use the techniques for Ring-LWE cryptography in [55] to give a tighter choice of parameters for desired security levels.
} 


\section{References}

1. Benny Applebaum, David Cash, Chris Peikert, and Amit Sahai. Fast cryptographic primitives and circular-secure encryption based on hard learning problems. In CRYPTO, pages 595-618. 2009.

2. László Babai. On Lovász' lattice reduction and the nearest lattice point problem. Combinatorica, 6(1):1-13, 1986.

3. Shi Bai and Steven D. Galbraith. An improved compression technique for signatures based on learning with errors. In CT-RSA, pages 28-47, 2014.

4. B. Barak, R. Impagliazzo, and A. Wigderson. Extracting randomness using few independent sources. SIAM Journal on Computing, 36(4):1095-1118, 2006.

5. E. Barker and A. Roginsky. Recommendation for the entropy sources used for random bit generation. Draft NIST Special Publication 800-90B, August 2012.

6. Mihir Bellare and Gregory Neven. Multi-signatures in the plain public-key model and a general forking lemma. In CCS, pages 390-399, 2006.

7. Mihir Bellare and Phillip Rogaway. Random oracles are practical: A paradigm for designing efficient protocols. In CCS, pages 62-73, 1993.

8. Mihir Bellare and Phillip Rogaway. Entity authentication and key distribution. In CRYPTO, volume 773, pages 232-249. 1994.

9. Joppe W. Bos, Craig Costello, Michael Naehrig, and Douglas Stebila. Post-quantum key exchange for the TLS protocol from the ring learning with errors problem. Cryptology ePrint Archive, Report 2014/599, 2014.

10. Z. Brakerski, C. Gentry, and V. Vaikuntanathan. Fully homomorphic encryption without bootstrapping. Innovations in Theoretical Computer Science, ITCS, pages 309-325, 2012.

11. Zvika Brakerski and Vinod Vaikuntanathan. Fully homomorphic encryption from Ring-LWE and security for key dependent messages. In CRYPTO, pages 505-524. 2011.

12. Christina Brzuska, Marc Fischlin, Nigel P. Smart, Bogdan Warinschi, and Stephen C. Williams. Less is more: relaxed yet composable security notions for key exchange. Int. J. Inf. Sec., 12(4):267-297, 2013.

13. Christina Brzuska, Marc Fischlin, Bogdan Warinschi, and Stephen C. Williams. Composability of bellare-rogaway key exchange protocols. In CCS, pages 51-62, 2011.

14. BSI. Advanced security mechanism for machine readable travel documents extended access control (eac). Technical Report (BSI-TR-03110) Version 2.05 Release Candidate, Bundesamt fuer Sicherheit in der Informationstechnik (BSI), 2010.

15. Ran Canetti and Hugo Krawczyk. Analysis of key-exchange protocols and their use for building secure channels. In EUROCRYPT, pages 453-474. 2001.

16. Ran Canetti and Hugo Krawczyk. Security analysis of IKEs signature-based key-exchange protocol. In CRYPTO, pages 143-161. 2002.

17. Lily Chen. Practical impacts on qutumn computing. Quantum-Safe-Crypto Workshop at the European Telecommunications Standards Institute, 2013. http://docbox.etsi.org/Workshop/2013/201309_CRYPTO/S05_DEPLOYMENT/ NIST_CHEN.pdf.

18. Yuanmi Chen and Phong Q. Nguyen. BKZ 2.0: Better lattice security estimates. In ASIACRYPT, pages 1-20, 2011.

19. Benny Chor and Oded Goldreich. Unbiased bits from sources of weak randomness and probabilistic communication complexity. In FOCS, pages 429-442, 1985.

20. Thomas H Cormen, Charles E Leiserson, Ronald L Rivest, Clifford Stein, et al. Introduction to algorithms, volume 2. MIT press Cambridge, 2001.

21. Özgür Dagdelen, Rachid El Bansarkhani, Florian Göpfert, Tim Güneysu, Tobias Oder, Thomas Pöppelmann, Ana Helena Snchez, and Peter Schwabe. High-speed signatures from standard lattices. In to appear at LATINCRYPT. 2014.

22. Özgür Dagdelen and Marc Fischlin. Security analysis of the extended access control protocol for machine readable travel documents. In ISC, pages 54-68, 2010.

23. Özgür Dagdelen, Marc Fischlin, Tommaso Gagliardoni, Giorgia Azzurra Marson, Arno Mittelbach, and Cristina Onete. A cryptographic analysis of OPACITY - (extended abstract). In ESORICS, pages 345-362, 2013.

24. Jean Paul Degabriele, Victoria Fehr, Marc Fischlin, Tommaso Gagliardoni, Felix Günther, Giorgia Azzurra Marson, Arno Mittelbach, and Kenneth G. Paterson. Unpicking PLAID - a cryptographic analysis of an ISO-standards-track authentication protocol. Cryptology ePrint Archive, Report 2014/728, 2014.

25. Tim Dierks. The transport layer security (TLS) protocol version 1.2. 2008.

26. W. Diffie and M. Hellman. New directions in cryptography. Information Theory, IEEE Transactions on, 22(6):644 - 654, nov 1976.

27. Jintai Ding, Xiang Xie, and Xiaodong Lin. A simple provably secure key exchange scheme based on the learning with errors problem. Cryptology ePrint Archive, Report 2012/688, 2012.

28. Yevgeniy Dodis, Rosario Gennaro, Johan Håstad, Hugo Krawczyk, and Tal Rabin. Randomness extraction and key derivation using the CBC, Cascade and HMAC modes. In CRYPTO, pages 494-510. 2004. 
29. Léo Ducas and Alain Durmus. Ring-LWE in polynomial rings. In PKC, pages 34-51, 2012.

30. Lo Ducas, Alain Durmus, Tancrde Lepoint, and Vadim Lyubashevsky. Lattice signatures and bimodal Gaussians. In CRYPTO, pages 40-56. 2013.

31. Alan Freier. The SSL protocol version 3.0. http://wp. netscape. com/eng/ssl3/draft302. txt, 1996.

32. Atsushi Fujioka, Koutarou Suzuki, Keita Xagawa, and Kazuki Yoneyama. Strongly secure authenticated key exchange from factoring, codes, and lattices. In PKC, pages 467-484. 2012.

33. Atsushi Fujioka, Koutarou Suzuki, Keita Xagawa, and Kazuki Yoneyama. Practical and post-quantum authenticated key exchange from one-way secure key encapsulation mechanism. In ASIACCS, pages 83-94, 2013.

34. Rosario Gennaro and Victor Shoup. A note on an encryption scheme of Kurosawa and Desmedt. Cryptology ePrint Archive, Report 2004/194, 2004.

35. Craig Gentry, Shai Halevi, and NigelP. Smart. Homomorphic evaluation of the AES circuit. In CRYPTO, pages 850-867. 2012.

36. Florian Giesen, Florian Kohlar, and Douglas Stebila. On the security of TLS renegotiation. In CCS, pages 387-398, 2013.

37. Shafi Goldwasser, Yael Tauman Kalai, Chris Peikert, and Vinod Vaikuntanathan. Robustness of the learning with errors assumption. In Innovations in Computer Science, pages 230-240, 2010.

38. Tim Güneysu, Vadim Lyubashevsky, and Thomas Pöppelmann. Practical lattice-based cryptography: A signature scheme for embedded systems. In CHES, pages 530-547, 2012.

39. Dan Harkins, Dave Carrel, et al. The internet key exchange (IKE). Technical report, RFC 2409, november, 1998.

40. Jeffrey Hoffstein, Jill Pipher, John M. Schanck, Joseph H. Silverman, and William Whyte. Practical signatures from the partial fourier recovery problem. In ACNS, pages 476-493, 2014.

41. ISO/IEC. 11770-3:2008 information technology - security techniques - key management - part 3: Mechanisms using asymmetric techniques.

42. Tibor Jager, Florian Kohlar, Sven Schäge, and Jörg Schwenk. On the security of TLS-DHE in the standard model. In CRYPTO, pages 273-293. 2012.

43. Jonathan Katz and Vinod Vaikuntanathan. Smooth projective hashing and password-based authenticated key exchange from lattices. In ASIACRYPT, pages 636-652. 2009.

44. Charlie Kaufman, Paul Hoffman, Yoav Nir, and Pasi Eronen. Internet key exchange protocol version 2 (IKEv2). Technical report, RFC 5996, September, 2010.

45. Hugo Krawczyk. SIGMA: The 'SIGn-and-MAc' approach to authenticated Diffie-Hellman and its use in the IKE protocols. In CRYPTO, pages 400-425. 2003.

46. Hugo Krawczyk. HMQV: A high-performance secure Diffie-Hellman protocol. In CRYPTO, pages 546-566. 2005.

47. Hugo Krawczyk, KennethG. Paterson, and Hoeteck Wee. On the security of the TLS protocol: A systematic analysis. In CRYPTO, pages 429-448. 2013.

48. Brian A. LaMacchia, Kristin E. Lauter, and Anton Mityagin. Stronger security of authenticated key exchange. In ProvSec, pages 1-16, 2007.

49. Xinyu Lei and Xiaofeng Liao. NTRU-KE: A lattice-based public key exchange protocol. Cryptology ePrint Archive, Report 2013/718, 2013.

50. Richard Lindner and Chris Peikert. Better key sizes (and attacks) for LWE-based encryption. In CT-RSA, pages 319-339. 2011.

51. Mingjie Liu and Phong Q. Nguyen. Solving BDD by enumeration: An update. In CT-RSA, pages 293-309. 2013.

52. Vadim Lyubashevsky. Lattice signatures without trapdoors. In David Pointcheval and Thomas Johansson, editors, EUROCRYPT, pages 738-755. 2012.

53. Vadim Lyubashevsky, Daniele Micciancio, Chris Peikert, and Alon Rosen. SWIFFT: A modest proposal for FFT hashing. In FSE, pages 54-72. 2008.

54. Vadim Lyubashevsky, Chris Peikert, and Oded Regev. On ideal lattices and learning with errors over rings. In EUROCRYPT, pages 1-23. 2010.

55. Vadim Lyubashevsky, Chris Peikert, and Oded Regev. A toolkit for Ring-LWE cryptography. In EUROCRYPT, pages 35-54. 2013.

56. Nikos Mavrogiannopoulos, Frederik Vercauteren, Vesselin Velichkov, and Bart Preneel. A cross-protocol attack on the TLS protocol. In CCS, pages 62-72, 2012.

57. A. Menezes, M. Qu, and S. Vanstone. Some new key agreement protocols providing mutual implicit authentication. In $S A C$, 1995.

58. Daniele Micciancio and Oded Regev. Worst-case to average-case reductions based on gaussian measures. SIAM J. Comput., 37:267-302, 2007.

59. Chris Peikert. Public-key cryptosystems from the worst-case shortest vector problem: extended abstract. In STOC, pages 333-342, 2009. 
60. Chris Peikert. An efficient and parallel Gaussian sampler for lattices. In CRYPTO, pages 80-97. 2010.

61. Chris Peikert. Lattice cryptography for the Internet. Cryptology ePrint Archive, Report 2014/070, 2014.

62. Chris Peikert and Brent Waters. Lossy trapdoor functions and their applications. In STOC, pages 187-196, 2008.

63. Claus-Peter Schnorr and M. Euchner. Lattice basis reduction: Improved practical algorithms and solving subset sum problems. Math. Program., 66:181-199, 1994.

64. P. Shor. Polynomial-time algorithms for prime factorization and discrete logarithms on a quantum computer. SIAM Journal on Computing, 26(5):1484-1509, 1997.

65. Damien Stehlé and Ron Steinfeld. Making NTRU as secure as worst-case problems over ideal lattices. In EUROCRYPT, pages 27-47. 2011.

66. L. Trevisan and S. Vadhan. Extracting randomness from samplable distributions. In FOCS, pages 32-, 2000.

67. Luca Trevisan. Extractors and pseudorandom generators. J. ACM, 48(4):860-879, July 2001.

68. Andrew Chi-Chih Yao and Yunlei Zhao. OAKE: A new family of implicitly authenticated Diffie-Hellman protocols. In $C C S$, pages 1113-1128, 2013. 